\title{
CO-CHANNEL INTERFERENCE SUPPRESSION IN MULTIHOP RELAY NETWORKS
}

by

\author{
Ahmed Mohamed Abd El-Salam Ahmed \\ B.Eng. Carleton University, Ottawa, 2005
}

\begin{abstract}
A THESIS SUBMITTED TO THE
Faculty of Graduate Studies and Research

IN PARTIAL FULFILLMENT OF THE REQUIREMENTS FOR THE DEGREE OF

Master of ApPlied Science
\end{abstract}

Ottawa-Carleton Institute for Electrical and Computer Engineering Department of Systems and Computer Engineering

Carleton University

Ottawa, Ontario

August 2007

(C) Ahmed Mohamed Abd El-Salam Ahmed, 2007 


$\begin{array}{ll}\begin{array}{l}\text { Library and } \\ \text { Archives Canada }\end{array} & \begin{array}{l}\text { Bibliothèque et } \\ \text { Archives Canada }\end{array} \\ \begin{array}{l}\text { Published Heritage } \\ \text { Branch }\end{array} & \begin{array}{l}\text { Direction du } \\ \text { Patrimoine de l'édition }\end{array} \\ \begin{array}{l}\text { 395 Wellington Street } \\ \text { Ottawa ON K1A ON4 }\end{array} & \begin{array}{l}\text { 395, rue Wellington } \\ \text { Ottawa ON K1A ON4 } \\ \text { Canada }\end{array}\end{array}$

Your file Votre référence ISBN: 978-0-494-33633-5 Our file Notre référence ISBN: 978-0-494-33633-5

NOTICE:

The author has granted a nonexclusive license allowing Library and Archives Canada to reproduce, publish, archive, preserve, conserve, communicate to the public by telecommunication or on the Internet, loan, distribute and sell theses worldwide, for commercial or noncommercial purposes, in microform, paper, electronic and/or any other formats.

The author retains copyright ownership and moral rights in this thesis. Neither the thesis nor substantial extracts from it may be printed or otherwise reproduced without the author's permission.
AVIS:

L'auteur a accordé une licence non exclusive permettant à la Bibliothèque et Archives Canada de reproduire, publier, archiver, sauvegarder, conserver, transmettre au public par télécommunication ou par l'Internet, prêter, distribuer et vendre des thèses partout dans le monde, à des fins commerciales ou autres, sur support microforme, papier, électronique et/ou autres formats.

L'auteur conserve la propriété du droit d'auteur et des droits moraux qui protège cette thèse. $\mathrm{Ni}$ la thèse ni des extraits substantiels de celle-ci ne doivent être imprimés ou autrement reproduits sans son autorisation.
In compliance with the Canadian

Privacy Act some supporting forms may have been removed from this thesis.

While these forms may be included in the document page count, their removal does not represent any loss of content from the thesis.
Conformément à la loi canadienne sur la protection de la vie privée, quelques formulaires secondaires ont été enlevés de cette thèse.

Bien que ces formulaires aient inclus dans la pagination, il n'y aura aucun contenu manquant.

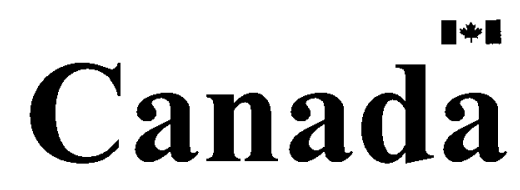




\begin{abstract}
In this thesis, we propose using iterative multi-user detectors for co-channel interference (CCI) suppression in wireless cellular systems. We demonstrate that a receiver with a single receive antenna is capable of detecting and cancelling a single in-band interferer that uses the same coding and modulation scheme as the desired signal. We investigate the benefits of using such an interference canceller in the mobile unit receiver in the downlink scenario of single-hop as well as two-hop networks. Results show significant improvements from iterative interference cancellation in terms of the average system throughput and outage probability. Benefits also include enabling the use of 3-sectored cells, where all sectors share the same frequency band, and the use of non-orthogonal channels for relaying.
\end{abstract}




\section{Acknowledgements}

I have had the great fortune to be supervised by Dr. Ian D. Marsland. I would like to take this opportunity to express my gratitude to him for his practical guidance, continuous support, and helpful contributions. My thanks and appreciations also go to Dr. Halim Yanikomeroglu for his continuous mentoring and support that helped me to complete this thesis. A special thanks goes to all my colleagues for many useful discussions we have had on the subject as well as continuous encouragement and support.

I wish to thank my family, especially my father, my mother, my wife, and my brother for their tremendous support, inspiration, encouragement, and love. 
Table of Contents

Abstract

Acknowledgements

Table of Contents $\quad$ iv

List of Tables $\quad$ vi

List of Figures vii

List of Symbols $\quad$ x

List of Acronyms xiv

Chapter 1: Introduction 1

1.1 Co-channel Interference . . . . . . . . . . . . . . . . . . 2

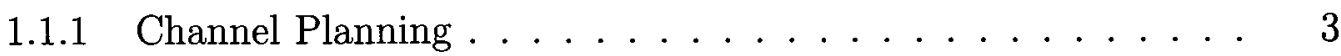

1.1 .2 Power Control . . . . . . . . . . . . . . . . . . . . . 3

1.1.3 Trunking.................... . . 4

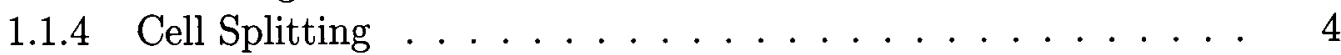

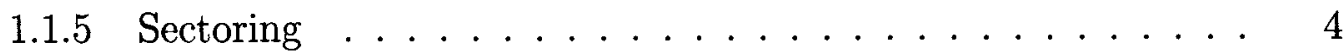

1.1.6 Iterative Interference Cancellation . . . . . . . . . . . 5

1.2 Wireless Relay Networks . . . . . . . . . . . . . . . . . . 7

1.3 Thesis Motivation and Contributions . . . . . . . . . . . . 9

1.4 Thesis Organization . . . . . . . . . . . . . . . . 10

Chapter 2: Interference Canceller Structure 12

2.1 Transmitter ....................... 13

2.1.1 Convolutional encoder . . . . . . . . . . . . . 14

2.1 .2 Bit interleaving . . . . . . . . . . . . . . . . . . . . 14

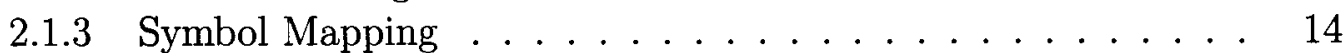

2.2 Channel Model . . . . . . . . . . . . . . . . . . . 16

2.2 .1 ISI channel . . . . . . . . . . . . . . . 16

2.2 .2 MIMO channel . . . . . . . . . . . . . . . . . . . . 17

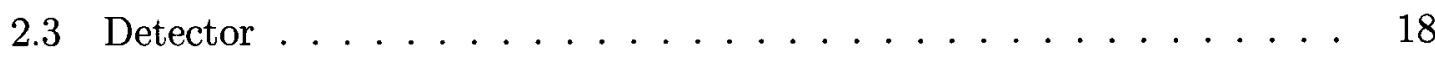

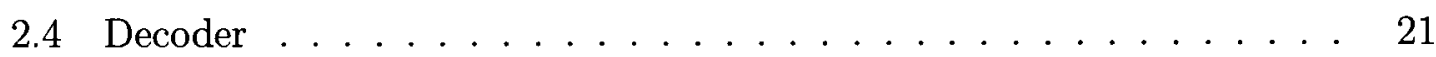

2.4.1 Metric Calculation Unit (MCU) . . . . . . . . . . 22

iv 
2.4.2 Metric De-interleaver . . . . . . . . . . . . . . . . . . . 22

2.4 .3 MAP decoder . . . . . . . . . . . . . . . . 23

2.5 Iterative Detection $\ldots \ldots \ldots \ldots \ldots \ldots \ldots \ldots$

2.5.1 Feedback interleaver . . . . . . . . . . . . . 24

2.5 .2 Soft symbol generator $\ldots \ldots \ldots \ldots \ldots \ldots$

2.5.3 Equivalent MIMO channel . . . . . . . . . . . . . 25

2.6 Link-level Simulation Results . . . . . . . . . . . . . . . 26

2.6.1 Simulation Results for $\left(N_{s}=2, N_{A}=1\right) \ldots \ldots \ldots \ldots 27$

2.6.2 Simulation Results for $\left(N_{s}=4, N_{A}=2\right) \ldots \ldots \ldots \ldots$

Chapter 3: $\quad$ Simulation Model 38

3.1 The Wireless Channel Propagation Model . . . . . . . . . . . . . 38

3.1 .1 Large-Scale Propagation Model . . . . . . . . . . . . . . . . 39

3.1.2 Small-Scale Propagation Model . . . . . . . . . . . . . 40

3.2 SINR Calculation . . . . . . . . . . . . . . . . . 41

3.3 Antenna Pattern . . . . . . . . . . . . . . . . . 42

3.3.1 Directional Antenna Pattern . . . . . . . . . . . . . . . . 44

3.3.2 Omnidirectional Gain Calculation . . . . . . . . . . . . 44

3.4 Cellular Layouts . . . . . . . . . . . . . . . . . . . . 46

3.5 Relaying Protocols . . . . . . . . . . . . . . . . 47

3.5.1 Relaying using ORCs . . . . . . . . . . . . . . . . 49

3.5.2 Relaying using nORCs . . . . . . . . . . . . . . 50

3.5.3 Probability of error calculation . . . . . . . . . . . 51

3.5.4 System Throughput and Outage Calculation . . . . . . . . 55

3.6 Simulation Algorithm and Parameters . . . . . . . . . . . 56

3.6.1 Environment Parameters and Assumptions . . . . . . . . 57

3.6 .2 Simulation Algorithm . . . . . . . . . . . . . . . 60

Chapter 4: Simulation Results 63

4.1 Single Hop . . . . . . . . . . . . . . . . . . . . . . 64

4.1 .1 Single-Cell Scenario . . . . . . . . . . . . . . . . . . . 64

4.1 .2 Multiple-Cell Scenario . . . . . . . . . . . . . . . . . 68

4.2 Two-Hop Relay Networks . . . . . . . . . . . . . . . . . . . 74

4.2.1 Multihop Using Orthogonal Relaying Channels (ORCs) . . . 74

4.2.2 Multihop Using non-Orthogonal Relaying Channels (nORCs) . 85

4.2.3 The Effect of the IC on Relaying Benefits . . . . . . . . . 98

Chapter 5: Conclusions and Future Work 106

5.1 Conclusions . . . . . . . . . . . . . . . . . . . 106

5.2 Future Work . . . . . . . . . . . . . . . . . . . . . . . . 108

$\begin{array}{ll}\text { References } & 110\end{array}$ 


\section{List of Tables}

3.1 Relaying protocol description, for relaying on ORCs. . . . . . . . . 49

3.2 Relaying protocol description, for relaying on nORCs. . . . . . . . 51

3.3 Cellular Layouts. . . . . . . . . . . . . . . . . . 59 


\section{List of Figures}

1.1 Example of frequency reuse concept, with a cluster size of seven cells. Cells with the same letter use the same channel group. Clusters are outlined in bold. . . . . . . . . . . . . . .

1.2 Illustration of antenna sectoring for a cluster size of seven, with three sectors per cell. Only two out of six co-channel cells interfere with the centre cell. . . . . . . . . . . . . . . . . 5

1.3 General structure of an iterative multi-user detector. . . . . . . . . 6

2.1 Block diagram of the MIMO system. . . . . . . . . . . . . . 13

2.2 Block diagram of a single transmitter chain. . . . . . . . . . . . . 13

2.3 Signal constellation for QPSK with Gray mapping. . . . . . . . . . 15

2.4 Block diagram of a MIMO channel. . . . . . . . . . . . . . . . . . . . . 19

2.5 Block diagram of the detector. . . . . . . . . . . . . . . 20

2.6 Structure of a bit de-interleaver. . . . . . . . . . . . . . . . . . 23

2.7 Block diagram of the detector with feedback. . . . . . . . . . . . . . . 24

2.8 Delay spread profile for SUI-5 channel. . . . . . . . . . . . . . 27

2.9 MIMO detector (w-IC) PER performance for $\left(N_{s}=2, N_{A}=1\right), \Delta_{1}=$ $0 \mathrm{~dB}, \Delta_{2} \in[-20,20] \mathrm{dB}$, SUI-5 channel model and QPSK. . . . . . 29

2.10 MIMO detector (w/o-IC) PER performance for $\left(N_{s}=2, N_{A}=1\right), \Delta_{1}$ $=0 \mathrm{~dB}, \Delta_{2} \in[-20,20] \mathrm{dB}$, SUI-5 channel model and QPSK. . . . . . 29

2.11 MIMO detector (w-IC) PER performance for $\left(N_{s}=2, N_{A}=1\right), \Delta_{1}=$ $0 \mathrm{~dB}, \Delta_{2}=0 \mathrm{~dB}$, SUI-5 channel model and QPSK. . . . . . .

2.12 MIMO detector (w-IC) PER performance for $\left(N_{s}=2, N_{A}=1\right), \Delta_{1}=$ $0 \mathrm{~dB}, \Delta_{2}=20 \mathrm{~dB}$, SUI-5 channel model and QPSK. . . . . . . .

2.13 MIMO detector (w-IC) PER performance for $\left(N_{s}=2, N_{A}=1\right), \Delta_{1}=$ $0 \mathrm{~dB}, \Delta_{2}=-20 \mathrm{~dB}$, SUI-5 channel model and QPSK. . . . . . . 31

2.14 MIMO detector PER performance for $\left(N_{s}=4, N_{A}=2\right), \Delta_{1}=0 \mathrm{~dB}$, $\Delta_{2}=0 \mathrm{~dB}, \Delta_{3}=0 \mathrm{~dB}, \Delta_{4}=0 \mathrm{~dB}$, SUI-5 channel model, and QPSK. 34

2.15 MIMO detector PER performance for $\left(N_{s}=4, N_{A}=2\right), \Delta_{1}=0 \mathrm{~dB}$, $\Delta_{2}=-10 \mathrm{~dB}, \Delta_{3}=-10 \mathrm{~dB}, \Delta_{4}=-10 \mathrm{~dB}$, SUI-5 channel model, and

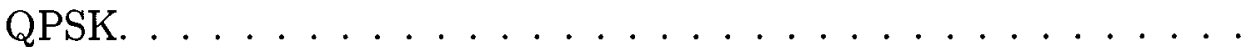

2.16 MIMO detector PER performance for $\left(N_{s}=4, N_{A}=2\right), \Delta_{1}=0 \mathrm{~dB}$, $\Delta_{2}=10 \mathrm{~dB}, \Delta_{3}=10 \mathrm{~dB}, \Delta_{4}=10 \mathrm{~dB}$, SUI-5 channel model, and QPSK. 35

2.17 MIMO detector PER performance for $\left(N_{s}=4, N_{A}=2\right), \Delta_{1}=0 \mathrm{~dB}$, $\Delta_{2}=5 \mathrm{~dB}, \Delta_{3}=10 \mathrm{~dB}, \Delta_{4}=15 \mathrm{~dB}$, SUI-5 channel model, and QPSK. 35 
2.18 MIMO detector PER performance for $\left(N_{s}=4, N_{A}=2\right), \Delta_{1}=0 \mathrm{~dB}$, $\Delta_{2}=-5 \mathrm{~dB}, \Delta_{3}=-10 \mathrm{~dB}, \Delta_{4}=-15 \mathrm{~dB}$, SUI-5 channel model, and QPSK.

2.19 MIMO detector PER performance for $\left(N_{s}=4, N_{A}=2\right), \Delta_{1}=0 \mathrm{~dB}$, $\Delta_{2}=-5 \mathrm{~dB}, \Delta_{3}=5 \mathrm{~dB}, \Delta_{4}=10 \mathrm{~dB}$, SUI-5 channel model, and QPSK. 36

2.20 MIMO detector PER performance for $\left(N_{s}=4, N_{A}=2\right), \Delta_{1}=0 \mathrm{~dB}$, $\Delta_{2}=5 \mathrm{~dB}, \Delta_{3}=-5 \mathrm{~dB}, \Delta_{4}=-10 \mathrm{~dB}$, SUI-5 channel model, and QPSK. 37

3.1 (a) Omnidirectional antenna pattern, (b) Directional antenna pattern. 43

3.2 BS antenna pattern for each sector, for $G_{\mathrm{DA}}=14 \mathrm{~dB}, \theta_{3 \mathrm{~dB}}=70^{\circ}$, and $A_{\max }=20 \mathrm{~dB} \ldots \ldots \ldots \ldots \ldots$

3.3 Hex cell layout, $C_{\mathrm{S}}=1$, centre cell is in hashed dark grey and interfering cells are in light grey. . . . . . . . . . . . . . . . . . 47

3.4 Hex cell layout, $C_{\mathrm{S}}=4$, centre cell is in hashed dark grey and interfering cells are in light grey. . . . . . . . . . . . . . . . 48

3.5 Relaying protocol for relaying on orthogonal channels (ORCs). . . . 50

3.6 Relaying protocol for relaying on non-orthogonal channels (nORCs). . 52

4.1 The average system throughput in (bpchu) for single-hop networks and

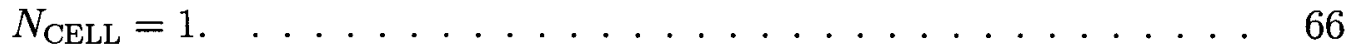

4.2 The throughput CDF at $R=500$ meters for single-hop networks with

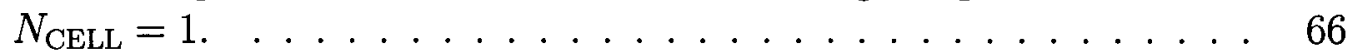

4.3 The outage probability for single-hop networks with $N_{\mathrm{CELL}}=1$. . . 67

4.4 The average system throughput in (bpchu) for single-hop, $N_{\mathrm{CELL}}=7$, and $C_{\mathrm{S}}=1$ networks. . . . . . . . . . . . . 70

4.5 The throughput CDF at $R=500$ meters for single-hop, $N_{\mathrm{CELL}}=7$,

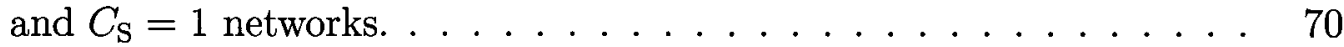

4.6 The outage probability for single-hop, $N_{\mathrm{CELL}}=7$, and $C_{\mathrm{S}}=1$ networks. 71

4.7 The average system throughput in (bpchu) for single-hop, $N_{\mathrm{CELL}}=7$, and $C_{\mathrm{S}}=4$ networks. . . . . . . . . . . . 71

4.8 The throughput CDF at $R=500$ meters for single-hop, $N_{\mathrm{CELL}}=7$, and $C_{\mathrm{S}}=4$ networks. . . . . . . . . . . . 72

4.9 The outage probability for single-hop, $N_{\mathrm{CELL}}=7$, and $C_{\mathrm{S}}=4$ networks. 72

4.10 System performance for two-hop networks using ORCs, $N_{\text {CELL }}=1$

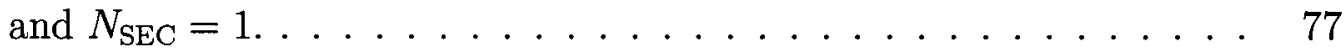

4.11 System performance for two-hop networks using ORCs, $N_{\mathrm{CELL}}=1$

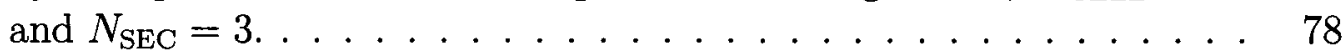

4.12 System performance for two-hop networks using ORCs, $N_{\mathrm{CELL}}=7$, $N_{\mathrm{SEC}}=1$, and $C_{\mathrm{S}}=1 \ldots \ldots \ldots 81$

4.13 System performance for two-hop networks using ORCs, $N_{\mathrm{R}}=6, N_{\mathrm{CELL}}=$

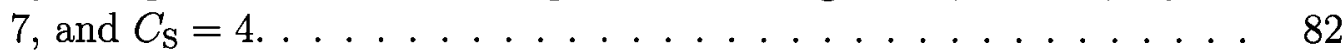

4.14 System performance for two-hop networks using ORCs, $N_{\text {CELL }}=7$, and $C_{\mathrm{S}}=1 \ldots \ldots \ldots \ldots \ldots \ldots$ 
4.15 System performance with perfect interference cancellation (PIC) for two-hop networks using ORCs, $N_{\mathrm{R}}=6, N_{\mathrm{CELL}}=7, N_{\mathrm{SEC}}=3$, and

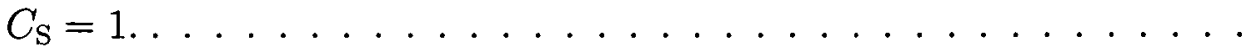

4.16 System performance for two-hop networks using nORCs, $N_{\mathrm{CELL}}=1$ and $N_{\mathrm{SEC}}=1 \ldots \ldots \ldots \ldots \ldots \ldots \ldots$

4.17 System performance for two-hop networks using nORCs, $N_{\mathrm{CELL}}=1$ and $N_{\mathrm{SEC}}=3 \ldots \ldots \ldots \ldots \ldots \ldots \ldots$

4.18 System performance for two-hop networks using nORCs, $N_{\mathrm{CELL}}=7$, $N_{\mathrm{SEC}}=1$, and $C_{\mathrm{S}}=1 \ldots \ldots \ldots \ldots$

4.19 System performance for two-hop networks using nORCs, $N_{\mathrm{CELL}}=7$, $N_{\mathrm{SEC}}=3$, and $C_{\mathrm{S}}=1 \ldots \ldots \ldots \ldots$

4.20 System performance with perfect interference cancellation (PIC) for two-hop networks using nORCs, $N_{\mathrm{CELL}}=7, N_{\mathrm{SEC}}=1$, and $C_{\mathrm{S}}=1$. .

4.21 System performance with perfect interference cancellation (PIC) for two-hop networks using nORCs, $N_{\mathrm{CELL}}=7, N_{\mathrm{SEC}}=3$, and $C_{\mathrm{S}}=1 . \quad .94$

4.22 System performance for two-hop networks using nORCs, $N_{\mathrm{CELL}}=7$, $N_{\mathrm{SEC}}=1$, and $C_{\mathrm{S}}=4 \ldots \ldots \ldots \ldots$

4.23 System performance for two-hop networks using nORCs, $N_{\mathrm{CELL}}=7$, $N_{\mathrm{SEC}}=3$, and $C_{\mathrm{S}}=4 \ldots \ldots \ldots \ldots$

4.24 System performance with perfect interference cancellation (PIC) for two-hop networks using nORCs, $N_{\mathrm{CELL}}=7, N_{\mathrm{SEC}}=3$, and $C_{\mathrm{S}}=4$.

4.25 System performance using IC, single vs. two-hop networks for $N_{\mathrm{CELL}}=$ 1 and $N_{\mathrm{SEC}}=3 \ldots \ldots \ldots \ldots \ldots \ldots$

4.26 System performance using IC, single vs. two-hop networks for $N_{\mathrm{CELL}}=$ $7, N_{\mathrm{SEC}}=1$, and $C_{\mathrm{S}}=1 \ldots \ldots \ldots 2 \ldots \ldots \ldots$

4.27 System performance using IC, single vs. two-hop networks for $N_{\mathrm{CELL}}=$ $7, N_{\mathrm{SEC}}=3$, and $C_{\mathrm{S}}=1 \ldots \ldots \ldots 103$

4.28 System performance using IC, single vs. two-hop networks for $N_{\mathrm{CELL}}=$ $7, N_{\mathrm{SEC}}=1$, and $C_{\mathrm{S}}=4 \ldots \ldots \ldots 104$

4.29 System performance using IC, single vs. two-hop networks for $N_{\mathrm{CELL}}=$ $7, N_{\mathrm{SEC}}=3$, and $C_{\mathrm{S}}=4 \ldots \ldots \ldots 10 \ldots \ldots$ 


\section{List of Symbols}

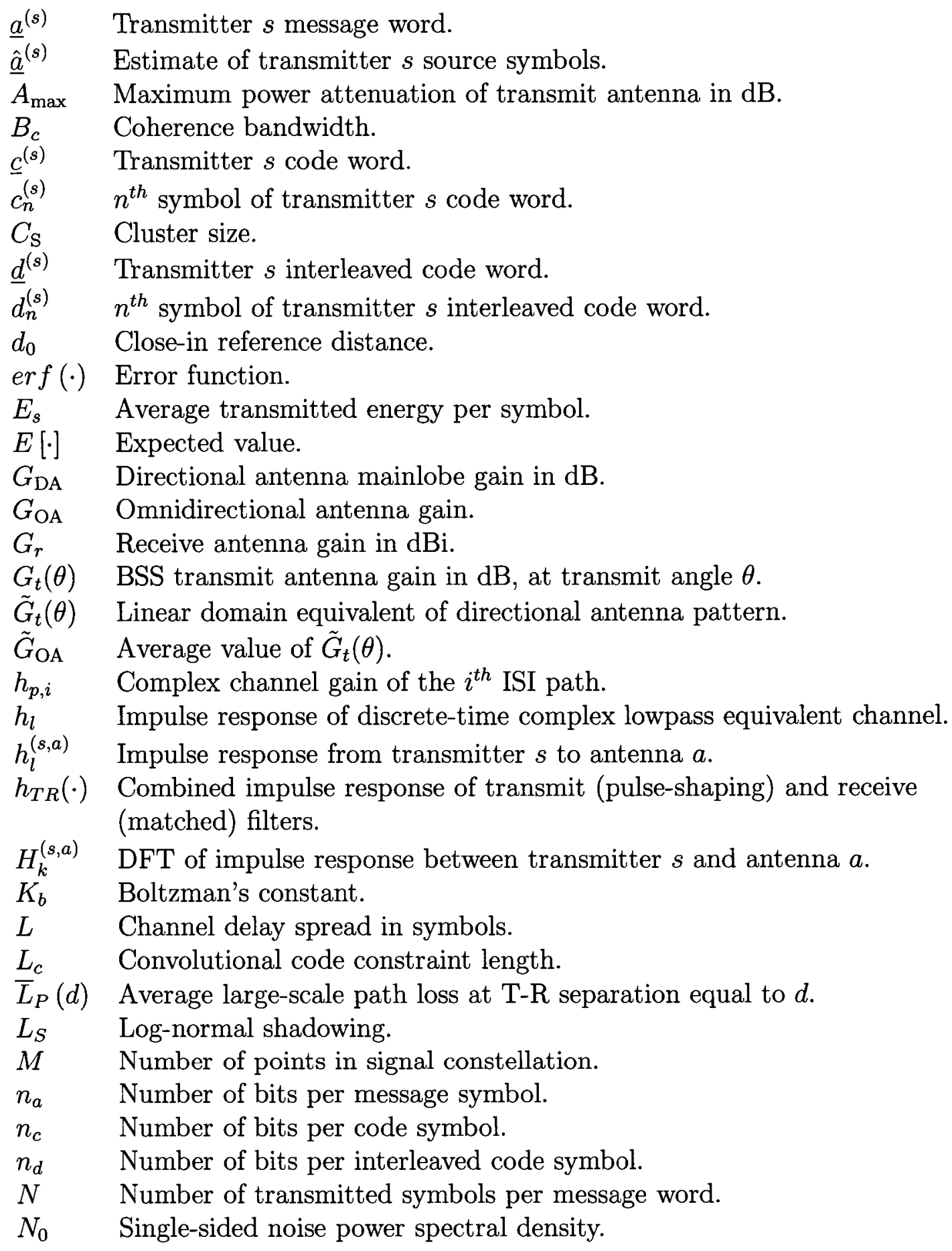


$N_{a} \quad$ Number of symbols per message word.

$N_{A} \quad$ Number of receive antennas.

$N_{\text {BSS }} \quad$ Number of interfering co-channel BSSs.

$N_{\text {CELL }} \quad$ Number of interfering co-channel cells.

$N_{c} \quad$ Number of symbols per code word.

$N_{F} \quad$ MU receiver noise figure in $\mathrm{dB}$.

$N_{p} \quad$ Number of signal paths between transmitter and receiver.

$N_{\mathrm{R}} \quad$ Number of available relays per cell.

$N_{\text {SEC }} \quad$ Number of BSSs per cell.

$N_{s} \quad$ Number of transmitters in the equivalent MIMO system.

$N_{T} \quad$ Thermal noise in dB.

$\overline{\mathrm{P}}_{e} \quad$ Average probability of packet error.

$\overline{\mathrm{P}}_{e, 1} \quad$ Average probability of packet error on the first hop.

$\overline{\mathrm{P}}_{e, 2} \quad$ Average probability of packet error on the second hop.

$\overline{\mathrm{PER}}_{i \mathrm{RSs}}^{(1)} \quad$ PER when $i$ RSs are interfering with the BSS-MU transmission on the first-hop.

$\mathrm{PER}_{i \mathrm{RSs}}^{(2)} \quad$ PER when $i$ RSs are interfering with the RS-MU transmission on the second-hop.

$P_{I} \quad$ Total interference power at receiver in $\mathrm{dB}$.

$P_{k}^{(s, a)} \quad$ Frequency-domain filter coefficient between transmitter $s$ and antenna $a$.

$P_{N} \quad$ AWGN power at receiver in $\mathrm{dB}$.

$P_{O} \quad$ Combined power of all interferers, other than primary interferers, at the receiver in $\mathrm{dB}$.

$P_{\text {OUT }} \quad$ Probability of outage.

$P_{p, i} \quad$ Power of $i^{\text {th }}$ path in $\mathrm{dB}$.

$P_{P} \quad$ Primary interferer or interferers total power in $\mathrm{dB}$.

$P_{r} \quad$ Received signal power in $\mathrm{dBm}$.

$P_{r, s} \quad$ Transmitter $s$ received signal power in $\mathrm{dBm}$.

$P_{S} \quad$ Desired signal power at receiver in $\mathrm{dB}$.

$P_{t} \quad$ Transmit signal power in $\mathrm{dBm}$.

$q_{0}^{(s)} \quad$ Filter coefficient of transmitter $s$ for bias term.

$R \quad$ Cell radius (size).

$\underline{r}^{(a)} \quad$ Symbols received at antenna $a$.

$r_{n}^{(a)} \quad n^{t h}$ received symbol at antenna $a$.

$R_{k}^{(a)} \quad$ DFT of received symbols at antenna $a$ at frequency $k$.

$\bar{R}_{k}^{(a)} \quad$ Expected value of $R_{k}^{(a)}$.

$\mathrm{SM}[\cdot] \quad$ Symbol mapping.

$T \quad$ Symbol period.

$T_{c} \quad$ Coherence time. 
$T_{n} \quad$ Time slot $n$.

$T_{K} \quad$ System temperature in kelvin.

$\underline{v}^{(s)} \quad$ Transmitter $s$ transmitted block.

$v_{n}^{(s)} \quad n^{\text {th }}$ symbol of transmitter $s$ transmitted block.

$\bar{v}_{n}^{(s)} \quad$ Expected value of $v_{n}^{(s)}$.

$V_{k}^{(s)} \quad$ DFT of $v_{n}^{(s)}$.

$w_{n}^{(a)} \quad n^{\text {th }}$ AWGN sample at antenna $a$.

$W_{k}^{(a)} \quad$ DFT of $w_{n}^{(a)}$.

$W \quad$ Transmission bandwidth.

$\underline{z}^{(s)} \quad$ Transmitter $s$ detector output.

$z_{n}^{(s)} \quad n^{\text {th }}$ symbol of transmitter $s$ detector output.

$\alpha_{s} \quad$ Transmitter $s$ signal attenuation factor.

$\Delta_{s} \quad$ Desired signal power to transmitter $s$ signal power ratio in $\mathrm{dB}$.

$\eta_{\mathrm{CDF}} \quad$ System throughput CDF.

$\eta_{\text {CELL }}$ Cell instantaneous throughput in bpchu.

$\eta_{\mathrm{MU}} \quad$ Mobile unit instantaneous throughput in bpchu.

$\eta_{\text {OUT }}$ Arbitrary chosen outage throughput in bpchu.

$\eta_{\text {SYS }} \quad$ System instantaneous throughput in bpchu.

$\bar{\eta}_{\text {SYS }} \quad$ Average system throughput in bpchu.

$\varsigma_{\mathrm{MU}} \quad \mathrm{MU}$ nominal throughput in bpchu.

SSYS System nominal throughput in bpchu.

$\gamma^{(s)} \quad$ Symbol metric produced by the MCU for transmitter $s$.

$\bar{\gamma}^{(s)} \quad$ Bit metrics produced by symbol-to-bit metrics converter for transmitter $s$.

$\dot{\chi}^{(s)} \quad$ De-interleaved bit metrics for transmitter $s$.

$\underline{\chi}^{(s)} \quad$ Symbol metrics produced by bit-to-symbol metrics converter for transmitter $s$.

$\psi^{(s)} \quad$ APPs of the code symbols produced by MAP decoder, input to feedback line, for transmitter $s$.

$\phi^{(s)} \quad$ Interleaved symbol metrics produced by feedback bit-to-symbol metrics converter for transmitter $s$.

$\Gamma \quad N_{A} \times N_{A}$ matrix.

$\delta_{m} \quad$ Kronecker delta function.

$\lambda \quad$ Carrier wavelength.

$\rho \quad$ Path loss exponent.

$\sigma_{p, i}^{2} \quad \mathcal{E}\left[\left|h_{p, i}\right|^{2}\right]$.

$\sigma_{v, n, s}^{2} \quad$ Variance of transmitter $s n^{\text {th }}$ transmitted symbol.

$\sigma_{S} \quad$ Standard deviation of log-normal shadowing. 
$\bar{\sigma}_{v, s}^{2} \quad$ Time-average variance of all transmitted symbols by transmitter $s$

$\bar{\sigma}_{z, s}^{2} \quad$ Time-average variance of $z_{n}^{(s)}$ conditioned on $d$

$\tau_{p, i} \quad$ Propagation delay of the $i^{\text {th }}$ ISI path.

$\tau_{p, i}^{(s, a)} \quad$ Propagation delay of the $i^{\text {th }}$ ISI path between transmitter $s$ and antenna a.

$\bar{\tau}_{p, i} \quad$ Average propagation delay of the $i^{\text {th }}$ ISI path.

$\theta \quad$ Horizontal BSS transmit angle.

$\theta_{3 \mathrm{~dB}} \quad 3 \mathrm{~dB}$ beamwidth of transmit antenna in degrees. 


\section{List of Acronyms}

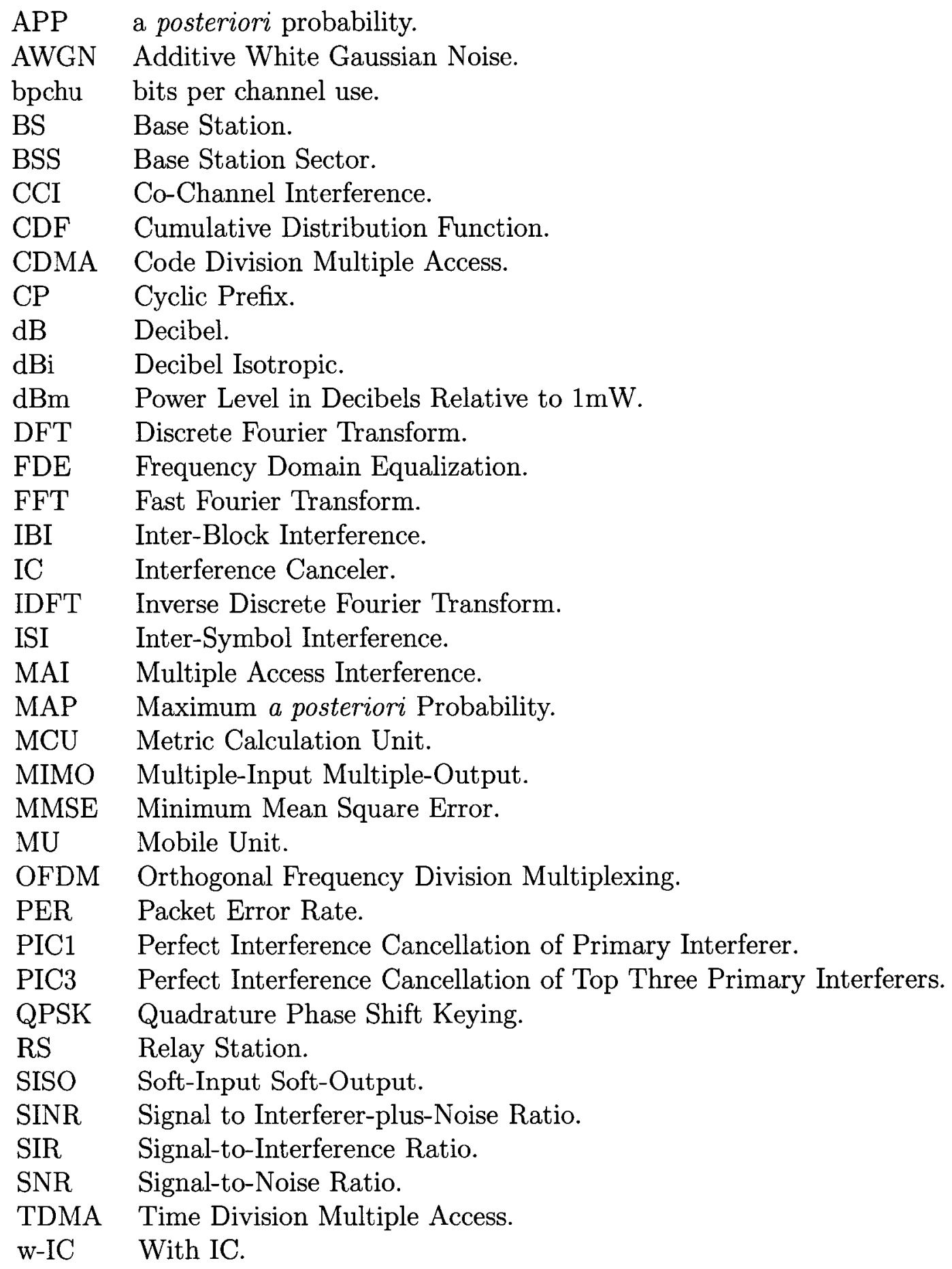


w/o-IC Without IC.

w/o-MAI Without MAI. 


\section{Chapter 1}

\section{Introduction}

Early wireless communication systems had a limited number of users, and hence the main objective of these systems was to support a large coverage area. This was achieved by using a single highly-mounted high-power transmitter antenna which provided very good coverage. However, these high-power transmitters made reusing the frequency band in nearby areas difficult, because doing so would result in very severe interference. With the increasing demand for mobile services, wireless networks had to support higher user capacity, which led to a spectral congestion problem.

The cellular concept, a system-level idea, was a major breakthrough in addressing this problem, where this single high power transmitter (large cell), was replaced with many low power transmitters (small cells). Each cell covers only a small portion of the service area, and is allocated a portion of the total number of available channels in the system, with neighbouring cells allocated different channel groups. The group of cells over which the total set of channels is distributed form a cluster, where no two cells in a cluster share the same channel group. The number of cells that comprise a cluster is known as the cluster size. If the service area contains more than one cluster, then more than one cell in the area will use the same channel group, resulting in frequency reuse, where the frequency reuse factor is defined as the reciprocal of the cluster size. These cells are called co-channel cells, and the interference between signals from these cells is called co-channel interference (CCI) [1,2]. 


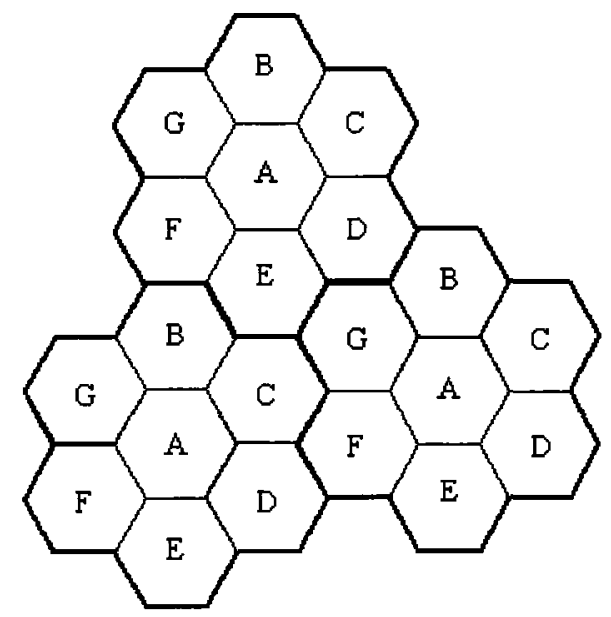

Figure 1.1: Example of frequency reuse concept, with a cluster size of seven cells. Cells with the same letter use the same channel group. Clusters are outlined in bold.

\subsection{Co-channel Interference}

Figure 1.1 illustrates the frequency reuse concept for a cluster size of seven, where cells labelled with the same letter use the same channel group. The cells in the figure have a hexagonal shape, with base stations located in the centre of each cell. This is commonly used in the design of wireless systems, although in practise the actual coverage of each cell, known as the cell footprint, is determined by field measurements.

$\mathrm{CCI}$ is one of the major limiting factors of cellular communication system performance, because unlike thermal noise it cannot be overcome through higher transmit power, which will increase CCI for neighbouring co-channel cells. When the cell size and the total number of available channels are fixed, increasing the cluster size provides more separation between co-channel cells and hence less CCI. However, this also means fewer available channels per cell. So we see a tradeoff between user capacity 
(requires a small cluster size) and link quality (requires a large cluster size). For that reason, different techniques have been developed to increase the capacity of cellular systems while maintaining its link quality.

These techniques include channel planning, power control, trunking, cell splitting, and antenna sectoring. All of these techniques tend to avoid or decrease CCI through careful planning and arranging of system level radio resources. Recently, there has been interest in using multi-user detection techniques for CCI cancellation instead of avoidance. In the following we briefly describe the different methods that are used to mitigate the effects of $\mathrm{CCI}$, either by avoidance or cancellation.

\subsubsection{Channel Planning}

Channel planning includes carefully distributing the available set of channels among the different cells in the cluster to minimize CCI as much as possible. This is done after the design phase, which determines the optimum frequency reuse ratio or cluster size and the appropriate separation between adjacent co-channel cells. Assigning the channels to the different cells in the cluster involves a great deal of trial and error and field measurements due to the real-world difficulties of radiopropagation and the imperfect coverage region of each cell.

\subsubsection{Power Control}

This method is usually applied in the uplink between the mobile unit and the base station, where the transmit power level of every mobile is under constant control by the serving base station. This is done to ensure that each user transmits the smallest power necessary to maintain acceptable quality link, hence reducing interference to other co-channel users. Power control is especially important for CDMA spread 
spectrum systems where all users share the same radio channel.

\subsubsection{Trunking}

This method exploits the fact that users do not use the system all the time, to accommodate a large number of users using a relatively small number of channels per cell. When a user requests service, it is allocated a channel on a per-call basis, and upon the termination of the call the channel is returned to the pool of available channels. If no channels are available, then the user's request will be blocked. The blocking probability can be calculated according to the principles of trunking theory which was developed by Erlang in the late $19^{\text {th }}$ century [2].

\subsubsection{Cell Splitting}

If a cell becomes too congested with users, it can be subdivided into smaller cells, each with its own base station. A corresponding reduction in the transmit antenna height and power is needed to maintain reasonable CCI levels. Doing this increases the number of times channels are reused in a given service area. Drawbacks of this method include real-estate costs to install new base stations and more frequent handoffs across the smaller cells. Also, usually not all cells are split at the same time, making channel assignment and planning more complicated.

\subsubsection{Sectoring}

By using directional instead of omnidirectional antennas it is possible to divide each cell into sectors. Typically each sector uses a different subset of the channels allocated to the cell. Sectoring allows for a reduction in CCI while keeping the cell size and transmit power fixed. This is illustrated in Figure 1.2 for a cluster size of seven, 


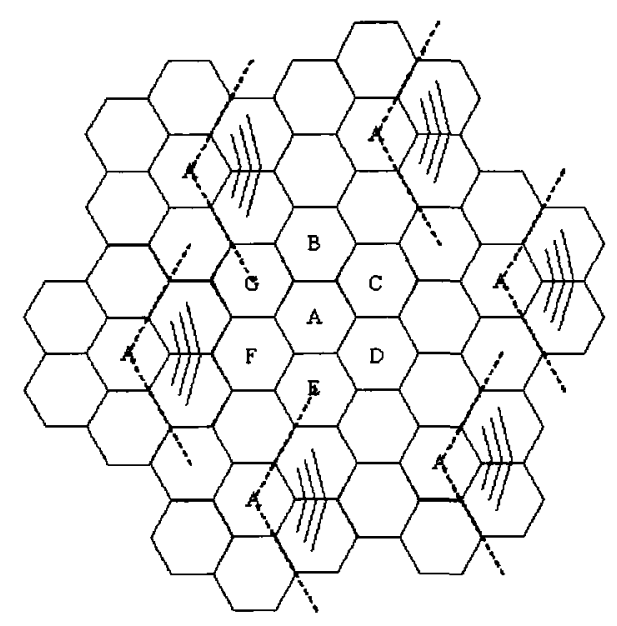

Figure 1.2: Illustration of antenna sectoring for a cluster size of seven, with three sectors per cell. Only two out of six co-channel cells interfere with the centre cell.

with three sectors per cell. If we consider the centre cell, labelled A, we see that there are only two first tier interferers which have sectors with antenna patterns radiating into the centre cell. This causes a significant reduction in CCI, thereby enabling the use of a smaller cluster size. Also, further CCI reduction can be achieved by down tilting the antennas such that the radiation pattern in the vertical plane has a notch at the nearest co-channel distance.

\subsubsection{Iterative Interference Cancellation}

The receiver structures built for multi-user detection are a promising technique for CCI mitigation. In the uplink of multiuser systems, several users transmit simultaneously over the same channel, so all the user's signals interfere with each other. Once a user's signal has been reliably detected, it can be subtracted from the received signal, there by removing its contribution to the overall interference. Because reliably detecting a user's signal in the presence of interference is difficult, iterative processing is typically employed [3-10]. 


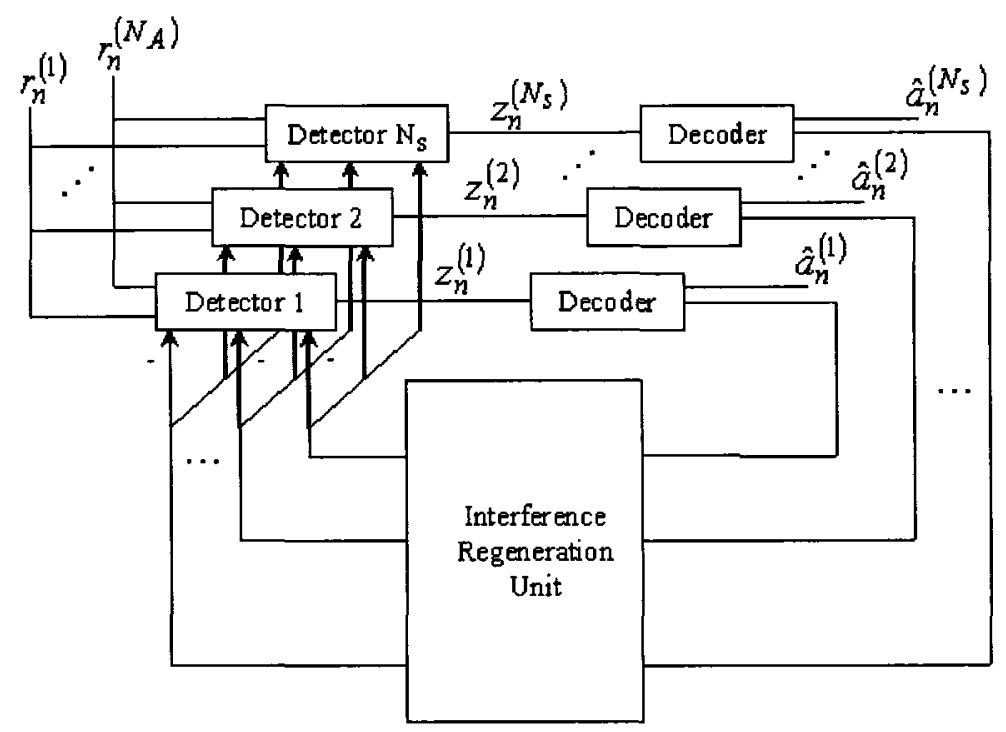

Figure 1.3: General structure of an iterative multi-user detector.

The structure of an iterative multi-user detector is shown in Figure 1.3. It consists of a bank of multi-user signal detectors followed by soft-input-soft-output (SISO) decoders whose outputs are fed back to an interference regeneration unit which builds a replica of the transmitted signals from each user, which can be subtracted from the received signal. Using channel coding and performing SISO decoding inside each detection iteration increases the reliability of the soft feedback, thereby reducing the error propagation phenomena between successive iterations $[4,5]$. As shown in the figure, multiple receive antennas can be incorporated in the multi-user detector. Additional antennas increase the number of users that can be successfully detected $[6,7]$

In a downlink scenario, the interference comes, not from other users, but from other base stations using the same channel group. Although the receiver (a single user) can receive the signals intended for the other users, it is not interested in them, so they are viewed as interferers. A multi-user detector can be employed by the user 
to detect all the signals, and cancel all but the desired one, so the multi-user detector is used as a co-channel interference canceller (IC).

In [8] space-time interference cancellers were introduced for CDMA systems using a bank of Rake receivers [11] that linearly combine signals from all antennas and all paths, followed by detection and interference subtraction where the receiver is assumed to have perfect channel estimates. To cope with unknown channels, adaptive space-time combiners were used in [10] instead of Rake receivers to avoid explicit need for channel estimation, where the adaptive combiners benefit from the iterative behaviour of the receiver.

Away from CDMA, in [12] Abe and Matsumoto proposed an iterative MIMO multi-user detector and time domain equalizer for use with frequency-selective channels to mitigate inter-symbol interference (ISI). Although very effective, this approach suffers from high computational complexity for channels with long delay spread, which will typically be the case for future high-speed applications.

To overcome this problem, in [9] Jie Lin introduced a MIMO multi-user detector that uses frequency domain equalization (FDE) $[13,14]$, and so requires much lower implementation complexity while being at least as effective at eliminating CCI as well as ISI.

\subsection{Wireless Relay Networks}

Wireless relay networks are currently receiving a great deal of interest in both academia and industry for the provisioning of ubiquitous high data rate coverage [15-17]. Unlike single-hop wireless systems, where the source and destination nodes communicate directly, in a relay network the source data may reach the destination over multiple hops. This is mainly important to extend coverage to hard-to-reach 
areas such as within buildings, or in valleys, tunnels or heavily shadowed locations where even the most advanced signal processing techniques do not work well [18].

Relaying techniques can be classified, according to the type of processing at the relay, into analog or digital relaying. In the former the relay amplifies and forwards the received analog signal together with noise without any decoding, while in the latter the relay decodes the received signal, re-encodes it and transmits it to the destination. Relaying can also be classified into peer-to-peer relaying, where relaying is performed by mobile user's equipment, and fixed relaying, where fixed relays installed by the service provider are responsible for relaying. In general, fixed relaying is more feasible and applicable for deployment as it depends upon service provider equipment not user equipment [19].

In a relaying network, an extra channel is needed for the relaying traffic. Different proposals have been made for the provision of this extra relaying channels, including using unlicensed bands, using channels exclusively reserved for relaying purposes, or searching for vacant channels and relaying only if one is available [20]. In [19], a fixed relaying-channel partition scheme was introduced where existing channels from neighbour cells in the network were reused for relaying purposes. However, this scheme only works for cluster size greater than one, while for a cluster size of one, channels from the same cell had to be used, which had a negative effect on the system performance due to excessive CCI. This demonstrates the need for efficient CCI cancellation techniques in relay networks to achieve higher user capacity through using smaller cluster sizes. 


\subsection{Thesis Motivation and Contributions}

Due to the increasing demand for mobile services, future wireless systems need to support higher user capacities than before. This requires resorting to resource efficient techniques such as smaller cluster sizes and using sectorized cells where all sectors use the same channel group. Moreover, in multihop wireless networks, an extra channel is needed for the relaying traffic. While using exclusively reserved channels for relaying is a waste of resources, reusing channels from neighbour cells deteriorate the link quality for other users especially for small cluster sizes as well as sectorized cells. Therefore, accommodating the relaying traffic on the same channel as the main transmission would be a very appealing choice. However all of these desired characteristics for future wireless systems involve a tremendous amount of cochannel interference, especially in the downlink scenario, where future mobile services go beyond traditional telephony to large data transfers such as wireless internet, multimedia, file transfer and downloading. This clearly demonstrates the need for efficient co-channel interference cancellation techniques to be employed at the mobile units in the downlink scenario of wireless systems.

Jie Lin introduced an iterative multi-user turbo detector/equalizer for MIMO channels in the frequency domain [9]. The implemented structure is effective in eliminating CCI as well as ISI. Frequency domain processing has the advantage of relatively low complexity growth with the increase of the channel delay spread compared to time domain processing. This is a significant advantage as the delay spread of future wireless system is expected to be very high, especially with the envisioned high data rates. This multi-user detector was proposed for use at the base station side in an uplink scenario, and it was able to detect twice as many users as the number 
of receive antennas at the receiver. However, the multi-user detector was only tested for synchronous users who have the same average power.

In this thesis we attempt to use Jie Lin's multi-user detector/equalizer as an iterative interference canceller for CCI suppression at the mobile unit (MU) side in the downlink scenario to make the previously mentioned resource efficient techniques more feasible. We study the benefits that can be achieved through detecting and cancelling CCI in both single-hop and two-hop networks. In both cases we look at single-cell as well as multiple-cells applications. However, the investigation is done for asynchronous interferers whose average power is not the same as each other. The study is done for a MU having a single receive antenna and hence it can detect and cancel the major interferer in the system.

Results show several benefits for using iterative CCI suppression in terms of average system throughput in bits per channel use (bpchu) as well as the outage probability of the system for single and multiple-cell applications, single and two-hop networks, and for different cluster sizes. Results are also shown for the perfect interference cancellation of the major interferer to measure the efficiency of the iterative canceller, and for the perfect cancellation of three major interferers to give an idea on the range of possible performance improvement if the $\mathrm{MU}$ were to have two receive antennas instead of only one.

\subsection{Thesis Organization}

This thesis is organized as follows. In Chapter 2 we describe the interference canceller (IC) structure including the transmitter, the modified channel model, and the receiver. Simulation results for the IC with different interferer power levels are also shown in this chapter. Chapter 3 discusses the system level simulation model 
including the wireless channel model, the cellular layouts investigated, the different relaying protocols studied, the throughput and outage calculation methods, the simulation parameters, and an outline of the simulation algorithm. In Chapter 4 we present the simulation results outlining the benefits of the IC first in single-hop networks then in two-hop networks. Finally, conclusions and possibilities for future work are presented in Chapter 5 . 


\section{Chapter 2}

\section{Interference Canceller Structure}

In this chapter the complete structure of the interference canceller (IC) proposed by Lin [9] is presented. The IC is comprised of a bank of multi-user detectors and soft-input-soft-output (SISO) decoders where each decoder's output is used as feedback information for improving its corresponding detector's estimates in an iterative manner.

The logical structure of a downlink scenario is shown in Figure 2.1. The desired transmitter along with $N_{s}-1$ interferers are each using one transmit antenna while the MU uses $N_{A}$ receive antennas to detect the multiple signals. So the result is a MIMO system with $N_{s}$ transmit antennas and $N_{A}$ receive antennas.

At each transmit entity the data are encoded using an error correcting code, then interleaved, and finally mapped onto complex symbols to be transmitted over the MIMO channel, where they are affected by multiple access interference (MAI) from other transmitters as well as inter-symbol interference (ISI) due to the channel delay spread. The MIMO channel output is then passed through a MIMO detector/equalizer to detect the multiple signals. Finally MAP decoding is used to estimate the transmitted messages.

The aim of this chapter is to provide a brief overview of the interference canceller, to discuss the modifications that were done to the channel model in [9], and finally 


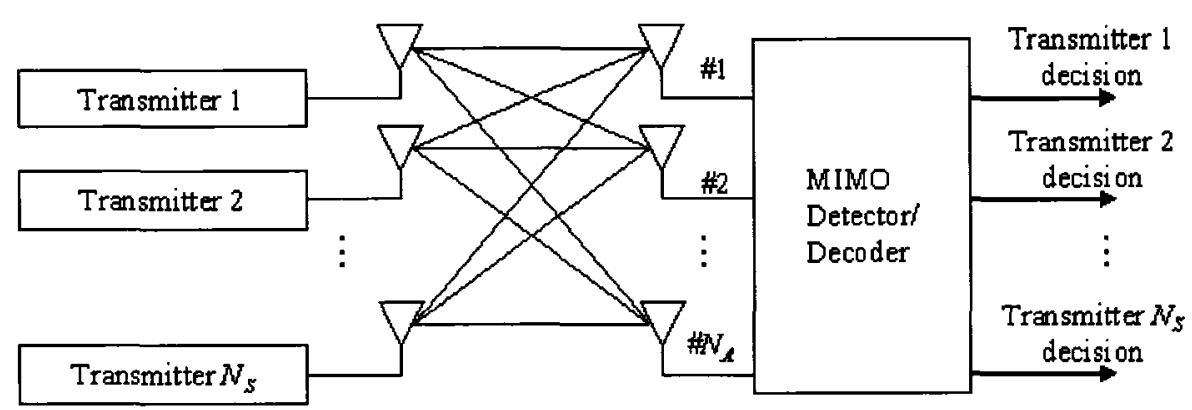

Figure 2.1: Block diagram of the MIMO system.

to show some results of the link level simulations for the modified channel model and discuss their implications.

\subsection{Transmitter}

A block diagram of a single transmit chain is shown in Figure 2.2. It is composed of four parts: The message source, the convolutional encoder, the interleaver, and the signal mapper. A message word $\underline{a}$ is composed of $N_{a}$ symbols and denoted by $\underline{a}=\left[a_{0}, a_{1}, \cdots, a_{N_{a}-1}\right]$ where $a_{n} \in\left\{0,1, \cdots, 2^{n_{a}-1}\right\}$ is the $n^{\text {th }}$ message symbol and $n_{a}$ is the number of bits per message symbol. The different message symbol values are all equally likely, and all message symbols are independent of each other.

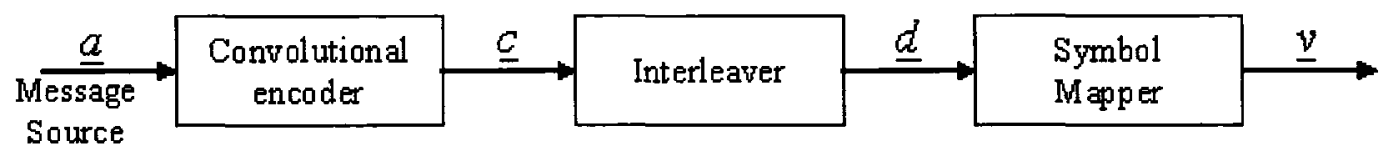

Figure 2.2: Block diagram of a single transmitter chain. 


\subsubsection{Convolutional encoder}

Each message word is then convolutionally encoded to produce a code word $\underline{c}=\left[c_{0}, c_{1}, \cdots, c_{N_{c}-1}\right]$ where each code symbol $c_{n} \in\left\{0,1, \cdots, 2^{n_{c}-1}\right\}$ is composed of $n_{c}$ bits. The code word length $N_{c}$ is equal to $N_{a}+\left(L_{c}-1\right)$ where $L_{c}$ is the constraint length of the code.

\subsubsection{Bit interleaving}

The code symbols are interleaved using a bit interleaver, where the code symbols $\underline{c}$ are converted into a bit stream, interleaved, and then converted back to symbols giving $\underline{d}=\left[d_{0}, d_{1}, \cdots, d_{N_{d}-1}\right]$ where $N_{d}$ is the number of code symbols after interleaving with the $n^{\text {th }}$ symbol given by $d_{n} \in\left\{0,1, \cdots, 2^{n_{d^{-1}}}\right\}$, where $n_{d}$ depends on the modulation scheme being used.

Random interleaving is employed where a pseudo-randomly selected permutation, assumed to be known at both the transmitter and receiver, is used to reorder the code bits. These mappings are different from one code word to another and most importantly they are different among all $N_{s}$ transmitters.

\subsubsection{Symbol Mapping}

The interleaved symbols, $\underline{d}$, are then mapped to points in an $M$-ary signal constellation, where $M=2^{n_{d}}$ is the number of points in the constellation. The signal mapper output is $\underline{v}=\left[v_{0}, v_{1}, \cdots, v_{N-1}\right]$, where $N=N_{d}$ is the number of transmitted symbols per message word. Signal mapping is done according to

$$
v_{n}=\operatorname{SM}\left[d_{n}\right]
$$




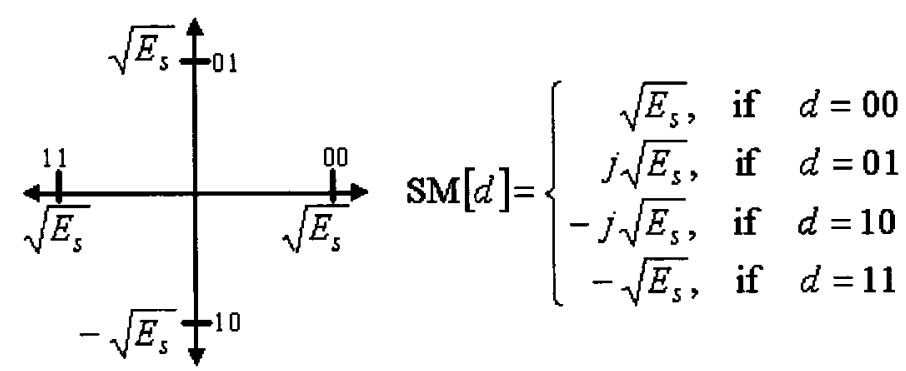

Figure 2.3: Signal constellation for QPSK with Gray mapping.

where SM [.] is the mapping between the digital symbol and the signal constellation points, based on the modulation scheme used. In this research the QPSK $(M=4)$ constellation shown in Figure 2.3 has been used for the link level simulations. The average transmitted energy per symbol is

$$
E_{s}=\frac{1}{M} \sum_{d=0}^{M-1}|\operatorname{SM}[d]|^{2}
$$

Before transmitting the modulated symbols, each data block is appended with a cyclic prefix (CP) of length $L$ symbols, where $L$ is the maximum channel impulse response length (in symbols). The $\mathrm{CP}$ is formed by transmitting the last $L$ symbols of the data block, before transmitting the data block, so $v_{-n}=v_{N-n}$ for all $n \in\{1,2, \cdots, L\}$. At the receiver the $\mathrm{CP}$ is removed and is not subjected to any processing. The importance of appending a CP is that it prevents inter-block interference (IBI) due to multipath propagation from previous blocks. It also transforms the linear convolution of the transmitted symbols with the channel impulse response into a circular convolution which can be computed efficiently in the discrete fourrier transform (DFT) domain [21]. 


\subsection{Channel Model}

\subsubsection{ISI channel}

Considering a single link between any transmitter $s$ and any receive antenna $a$, a discrete-time ISI channel can be used to describe the combination of the transmitter's pulse shaping filter and modulator, the analog multipath fading channel, the receiver's demodulator, matched filter (matched to the transmitted pulse shape), and a symbolrate sampler. The channel output at time $n$ can be expressed according to this discrete-time channel as

$$
r_{n}=\sum_{l=0}^{L} h_{l} v_{n-l}+w_{n}
$$

where $L$ is the channel delay spread, $v_{n-l}$ is the transmitted symbol at time $n-l$, $w_{n}$ is additive white Gaussian noise (AWGN), and $h_{l}$ is the discrete-time channel impulse response given by

$$
h_{l}=\sum_{i=0}^{N_{p}-1} h_{p, i} h_{\mathrm{TR}}\left(l T-\tau_{p, i}\right)
$$

where $N_{p}$ is the number of paths in between the transmitter and the receiver, $T$ is the symbol period, $\tau_{p, i}$ is the arrival time of the $i^{\text {th }}$ path relative to the first path, $h_{\mathrm{TR}}(\cdot)$ is the combined transmit-receive filter impulse response, and $h_{p, i}$ is the fading multiplicative factor of the $i^{\text {th }}$ path.

A subtle difference between the channel model investigated in this research and the model in [9] is that the path arrival time $\tau_{p, i}$ is not considered to be only integer multiples of the symbol period $T$. Rather, in this research $\tau_{p, i}$ is uniformly distributed in the range $\left[\bar{\tau}_{p, i}-\frac{T}{2}, \bar{\tau}_{p, i}+\frac{T}{2}\right]$ where $\bar{\tau}_{p, i}$ is an integer multiple of the symbol period. The latter scenario alleviates the restriction of requiring the different paths to arrive at exact integer multiples of the symbol period, which is not realistic especially in 
MIMO systems with $N_{s}$ and $N_{A} \geq 1$. This modification to the channel model used in [9] reflects the asynchronousity among the different interfering signals.

The fading factors $\left\{h_{p, i}\right\}$ are all independent and each has a complex Gaussian distribution, with a mean of zero and a normalized variance of

$$
\sigma_{p, i}^{2}=E\left[\left|h_{p, i}\right|^{2}\right]
$$

which is calculated for each path $i$ according to

$$
\sigma_{p, i}^{2}=\frac{10^{P_{p, i} / 10}}{\sum_{j=1}^{N_{p}} 10^{P_{p, j} / 10}}
$$

where $P_{p, i}$ is the power of the $i^{t h}$ path in $\mathrm{dB}$, as specified by the power delay profile of the channel model used. Finally, the AWGN has a single-sided noise power spectral density of $N_{0}$, so

$$
E\left[w_{n}\right]=0 \quad \text { and } \quad E\left[w_{n-m}^{*} w_{n}\right]=N_{0} \delta_{m}
$$

where $\delta_{m}=\left\{\begin{array}{lll}1 & \text { if } & m=0 \\ 0 & \text { if } & m \neq 0\end{array}\right.$ is the Kronecker delta function.

\subsubsection{MIMO channel}

In a MIMO system, all transmitted signals share the same time and frequency slot, so each transmitter creates multiple access interference (MAI) for all the other transmitters in addition to the (ISI) caused by the transmitter's own traffic. Each link between any given transmit antenna and any given receive antenna suffers from Rayleigh frequency selective fading, where the fading between different transmitters and receivers is uncorrelated.

While the iterative receiver in [9] was intended for the use at the BS side, where the $N_{s}$ transmitters were all assumed to be MUs with equal average received signal 
power, in this research the iterative receiver will be used as an IC on the MU side in the downlink scenario with $N_{s}-1$ interferers and one desired signal. The average received signal power from these interferers differ within themselves as well as from the desired transmitter. To model the difference in average received signal power among the different transmitters, let $s=1$ always be the transmitter of the desired signal, then the signal-to-interference ratio (SIR) parameter $\Delta_{s}$ given by

$$
\Delta_{s}=P_{r, 1}-P_{r, s}, \quad \forall s \in\left\{1,2, \cdots, N_{s}\right\}
$$

is the ratio of the received power $P_{r, 1}$ from the desired transmitter $(s=1)$ to the received power $P_{r, s}$ from transmitter $s$, in $\mathrm{dB}$. Therefore, the interferer power increases with the decrease of $\Delta_{s}$. In the linear domain, the SIR parameter translates to an attenuation factor $\alpha_{s}$ given by

$$
\alpha_{s}=\sqrt{10^{-\frac{\Delta_{s}}{10}}}
$$

which is equal to 1 for $s=1$.

Figure 2.4 shows a block diagram of the MIMO channel with $N_{s}$ transmit antennas and $N_{A}$ receive antennas. For $s \in\left\{1,2, \cdots, N_{s}\right\}$, transmitter $s$ transmits the symbols $\underline{v}^{(s)}=\left[v_{0}^{(s)}, v_{1}^{(s)}, \cdots, v_{N-1}^{(s)}\right]$, along with its cyclic prefix. For $a \in\left\{1,2, \cdots, N_{A}\right\}$, the $n^{\text {th }}$ sample from receiving antenna $a$ at time $n$ is

$$
r_{n}^{(a)}=\sum_{s=1}^{N_{s}} \sum_{l=0}^{L} \alpha_{s} h_{l}^{(s, a)} v_{n-l}^{(s)}+w_{n}^{(a)}
$$

\subsection{Detector}

The function of the detector is to generate an estimate $\underline{z}^{(s)}$ of the transmitted symbol sequence $\underline{v}^{(s)}$ for all $s \in\left\{1,2, \cdots, N_{s}\right\}$. This is done through linear filtering of the signals from each antenna then combining the output of the filters. Because 


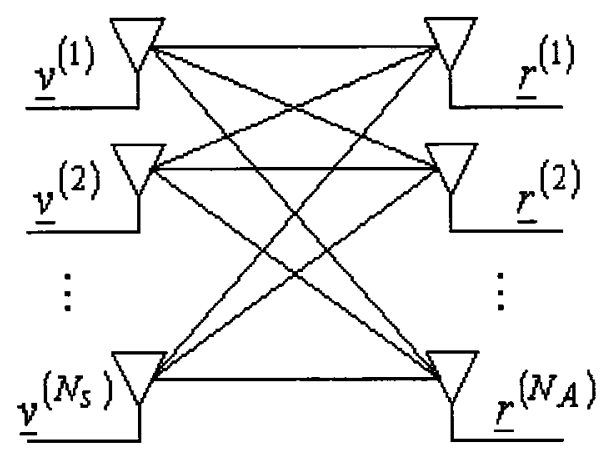

Figure 2.4: Block diagram of a MIMO channel.

of the cyclic prefix, the data symbols $\left\{v_{n}^{(s)}\right\}$ can be assumed to be periodic, i.e. $v_{m}^{(s)}=v_{m+N}^{(s)}$ for $m \in\{-L+1,-L+1, \cdots,-2,-1\}$, and hence linear convolution with the channel impulse response is equivalent to circular convolution. This enables filtering to be done efficiently in the frequency domain.

The block diagram for the detector responsible for detecting traffic from transmitter $s$ is shown in Figure 2.5. After discarding the cyclic prefix, an $N$-point DFT is applied to the $N$ received samples from each antenna. So for antenna $a$, the frequency domain received samples will be given by

$$
R_{k}^{(a)}=\sum_{s=1}^{N_{s}} \alpha_{s} H_{k}^{(s, a)} V_{k}^{(s)}+W_{k}^{(a)}, \quad \forall k \in\{0,1,2, \cdots, N-1\} .
$$

where $H_{k}^{(s, a)}$ is the $k^{t h}$ frequency component of the DFT of the channel impulse response between the $s^{t h}$ transmitter and the $a^{t h}$ receiver antenna, $V_{k}^{(s)}$ is the $k^{t h}$ frequency component of the DFT of the symbols $\left\{v_{n}^{(s)}\right\}$, and $W_{k}^{(a)}$ is the $k^{\text {th }}$ frequency components of the DFT of the noise samples $\left\{w_{n}^{(a)}\right\}$ at receiver antenna $a$.

Frequency domain filtering is done by multiplying each frequency component $R_{k}^{(a)}$ by the filter coefficient $P_{k}^{(s, a)}$. The filtered frequency components are then added, and applied to the IDFT operation. The output of the IDFT operation together with an 


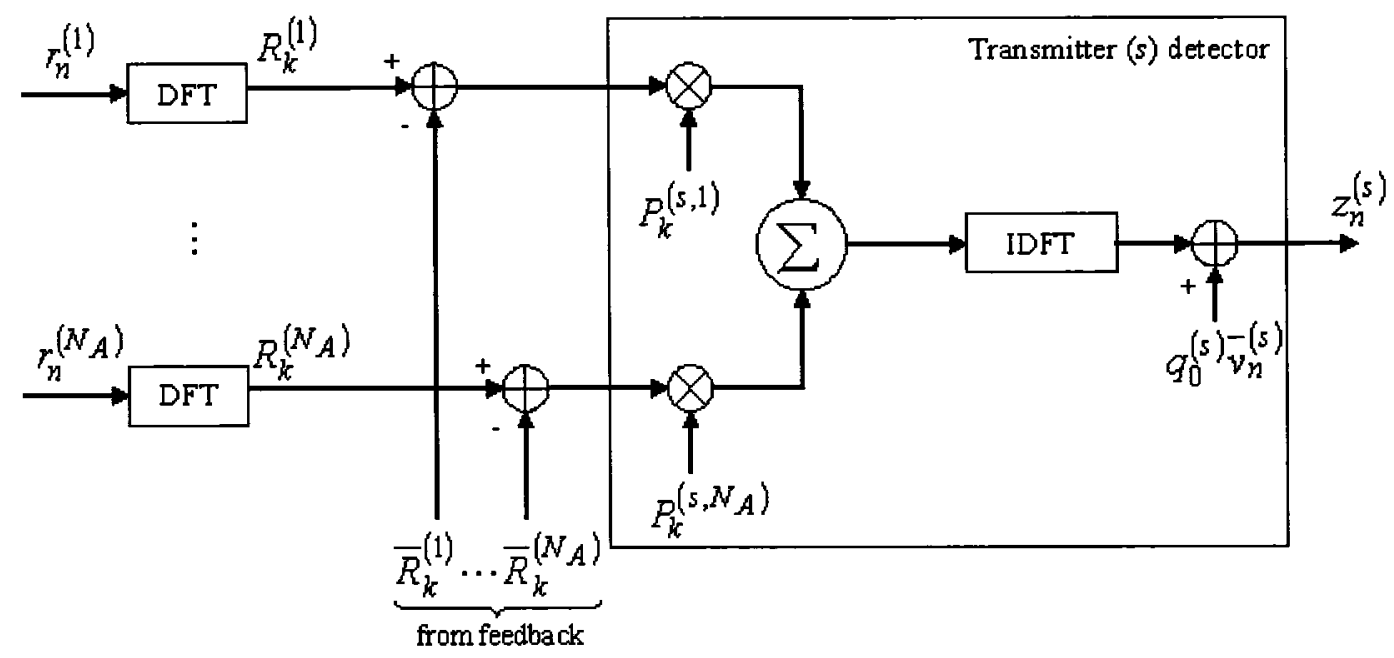

Figure 2.5: Block diagram of the detector.

anti-biasing term form the detector estimate for $v_{n}^{(s)}$, which is given by

$$
z_{n}^{(s)}=\frac{1}{N} \sum_{k=0}^{N-1} \sum_{a=1}^{N_{A}} P_{k}^{(s, a)}\left(R_{k}^{(a)}-\bar{R}_{k}^{(a)}\right) e^{j 2 \pi \frac{k n}{N}}+q_{0}^{(s)} \bar{v}_{n}^{(s)}
$$

where $\vec{R}_{k}^{(a)}$ is the expected value of $R_{k}^{(a)}$, which is obtained from the iterative detection feedback loop discussed in Section 2.5, and

$$
q_{0}^{(s)}=\frac{1}{N} \sum_{k=0}^{N-1} \sum_{a=1}^{N_{A}} P_{k}^{(s, a)} H_{k}^{(s, a)} .
$$

The filter coefficients $\left\{P_{k}^{(s, a)}\right\}$ are determined according to the minimum mean square error (MMSE) criterion, by solving $N$ sets of linear equations, one set for each DFT point. Each set consists of the $N_{A}$ equations given by

$$
\left[\begin{array}{cccc}
\Gamma_{k}^{(1,1)} & \Gamma_{k}^{(1,2)} & \cdots & \Gamma_{k}^{\left(1, N_{A}\right)} \\
\Gamma_{k}^{(2,1)} & \Gamma_{k}^{(2,2)} & \cdots & \Gamma_{k}^{\left(2, N_{A}\right)} \\
\vdots & \vdots & \ddots & \vdots \\
\Gamma_{k}^{\left(N_{A}, 1\right)} & \Gamma_{k}^{\left(N_{A}, 2\right)} & \cdots & \Gamma_{k}^{\left(N_{A}, N_{A}\right)}
\end{array}\right]\left[\begin{array}{c}
P_{k}^{(s, 1)} \\
P_{k}^{(s, 2)} \\
\vdots \\
P_{k}^{\left(s, N_{A}\right)}
\end{array}\right]=\left[\begin{array}{c}
H_{k}^{(s, 1) *} \\
H_{k}^{(s, 2) *} \\
\vdots \\
H_{k}^{\left(s, N_{A}\right) *}
\end{array}\right],
$$


for all $k \in\{0,1, \cdots, N-1\}$, where $*$ denotes complex conjugate, and

$$
\Gamma_{k}^{(b, a)}=\sum_{s=1}^{N_{s}} H_{k}^{(s, a)} H_{k}^{(s, b) *} \bar{\sigma}_{v, s}^{2}
$$

The time averaged variance of all $N$ symbols $\left(\bar{\sigma}_{v, s}^{2}\right)$ is given by

$$
\bar{\sigma}_{v, s}^{2}=\frac{1}{N} \sum_{n=0}^{N-1} \sigma_{v, n, s}^{2}
$$

where

$$
\sigma_{v, n, s}^{2}=E\left[\left|v_{n}^{(s)}-\bar{v}_{n}^{(s)}\right|^{2}\right]=\sum_{d=0}^{M-1}|\operatorname{SM}[d]|^{2} \operatorname{Pr}\left\{d_{n}^{(s)}=d\right\}-\left|\bar{v}_{n}^{(s)}\right|^{2}
$$

is the variance of the $n^{\text {th }}$ transmitted symbol from transmitter $s . \operatorname{Pr}\left\{d_{n}^{(s)}=d\right\}$ is the arbitrary a priori probability distribution of $d_{n}^{(s)}$, which is assumed to be potentially different for all $n$ and $s$. The significance of this assumption will be explained in the iterative detection section. $\bar{v}_{n}^{(s)}$ is the expected value of the $n^{\text {th }}$ symbol from transmitter $s$, which is obtained from the iterative detection feedback loop as well.

Theoretically it is better to use $\sigma_{v, n, s}^{2}$ instead of $\bar{\sigma}_{v, s}^{2}$ in the calculation of the filter coefficients. However, doing so would greatly increase the filtering complexity as the filter coefficients will be different for each symbol $n$. Moreover, in [9] it is shown that this sub optimality is not a great concern. The reader is referred to the previous source for the detailed derivation of the filter coefficients based on the MMSE criterion.

\subsection{Decoder}

A MAP decoder is used at the receiver to decode the convolutionally encoded message words. The MAP decoder calculates the a posteriori probabilities (APPs) of the message symbols, $\operatorname{Pr}\left\{a_{n}^{(s)}=a \mid \underline{z}^{(s)}\right\}$, and eventually uses a decision device to 
make hard decisions on these APPs. To accomplish this a metric calculation unit $(\mathrm{MCU})$ is needed to prepare the branch metrics needed for MAP decoding. Also a de-interleaver is used after the MCU to restore the proper order of bits corresponding to the changes made by the interleaver at the transmitter.

\subsubsection{Metric Calculation Unit (MCU)}

The MAP decoder evaluates the likelihood function $f\left(z_{n} \mid d_{n}^{(s)}=d\right)$ of the detector output, $z_{n}^{(s)}$, conditioned on the transmitted symbols, $d_{n}^{(s)}$, as the metrics. The symbol metric, $\gamma_{n}^{(s)}(d)$, calculated by the MCU for a symbol $d$ can be written as

$$
\gamma_{n}^{(s)}(d)=f\left(z_{n} \mid d_{n}^{(s)}=d\right)
$$

for each $d \in\{0,1, \cdots, M-1\}$ and $n \in\{0,1, \cdots, N-1\}$. Therefore, for each detector output sample, a set of $M$ metrics are calculated by the MCU.

\subsubsection{Metric De-interleaver}

The function of the de-interleaver is to restore the proper order of symbols that was changed by the interleaver at the transmitter side. This also provides the benefit of breaking any error bursts produced by the detector. Since bit interleaving was used at the transmitter, the symbol metrics, $\left\{\underline{\gamma}^{(s)}\right\}$, produced by the MCU have to be first converted into bit metrics, $\left\{\underline{\hat{\gamma}}^{(s)}\right\}$, which will be applied to the bit deinterleaver producing a stream of bit metrics in the proper order, $\left\{\underline{\hat{\chi}}^{(s)}\right\}$, that is converted back again to the symbol metrics, $\left\{\underline{\chi}^{(s)}\right\}$, which are applied to the MAP decoder. This is illustrated in Figure 2.6. 


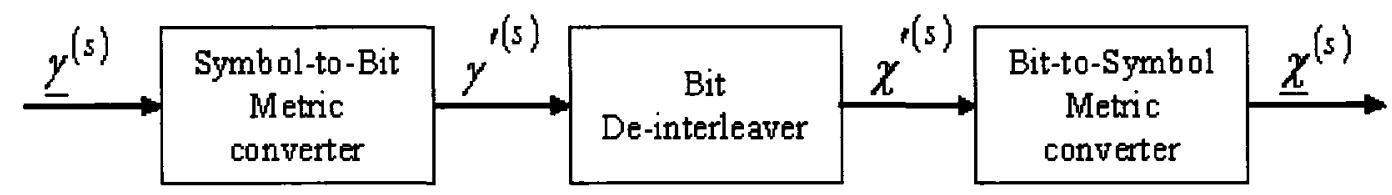

Figure 2.6: Structure of a bit de-interleaver.

\subsubsection{MAP decoder}

In this research a maximum a posteriori probability (MAP) decoder is used to decode the convolutional code used at the transmitter. MAP decoding is based on minimizing the probability of a message symbol error, by calculating the a posteriori probabilities (APPs) of the message symbols and then making hard decisions on these APPs. The APP that message symbol $a_{n}^{(s)}$ is equal to message symbol $a \in$ $\left\{0,1, \cdots, 2^{n_{a}}-1\right\}$ is $\operatorname{Pr}\left\{a_{n}^{(s)}=a \mid \underline{z}^{(s)}\right\}$ which can be calculated efficiently using the BCJR algorithm [22].

After calculating the APP's for $a_{n}^{(s)}$, the most probable transmitted symbol at time $n$ is selected according to $\widehat{a}_{n}^{(s)}=\arg \max _{a} \operatorname{Pr}\left\{a_{n}^{(s)}=a \mid \underline{z}^{(s)}\right\}$, where $\widehat{a}_{n}^{(s)}$ is the decoder estimate of $a_{n}^{(s)}$.

\subsection{Iterative Detection}

The previous sections explained a single iteration of interference cancellation and decoding. Much better results can be achieved if the decoder output is fed back to the detectors where it is used to better cancel the interference in an iterative manner. Figure 2.7 shows a block diagram of the receiver with feedback from the decoder bank. In this section the feedback loop, composed of a feedback interleaver, a soft symbol generator, and an equivalent MIMO channel, is discussed. 


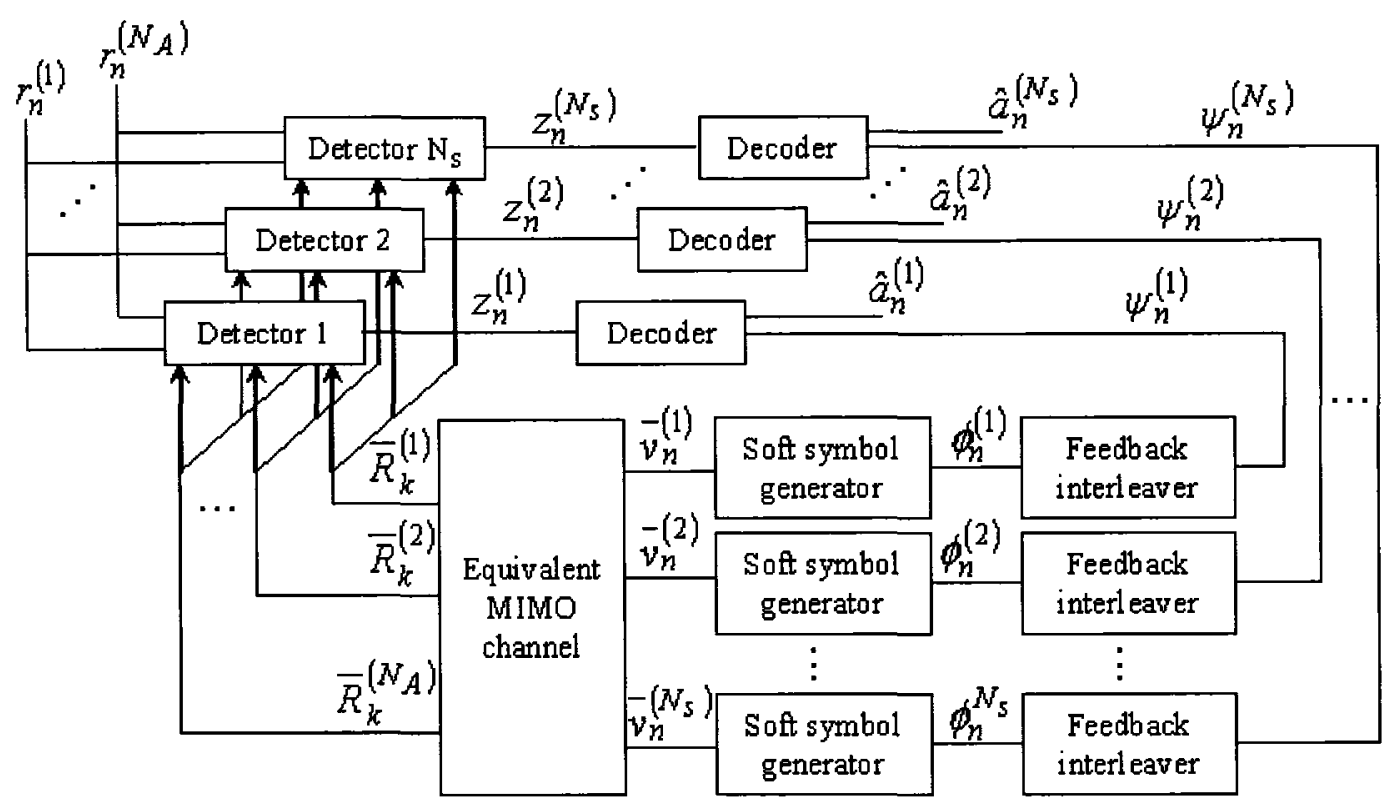

Figure 2.7: Block diagram of the detector with feedback.

\subsubsection{Feedback interleaver}

At the end of each iteration the decoder, in addition to calculating APPs of the message symbols $\left(\operatorname{Pr}\left\{a_{n}^{(s)}=a \mid \underline{z}^{(s)}\right\}\right)$, calculates the APPs of the code symbols $\psi_{n}^{(s)}(c)=\operatorname{Pr}\left\{c_{n}^{(s)}=c \mid \underline{z}^{(s)}\right\}$, for all $c \in\left\{0,1, \cdots, 2^{n_{c}-1}\right\}$ and $n \in\left\{0,1, \cdots, N_{c}-1\right\}$. This output of the MAP decoder is the input to the feedback interleaver which, similar to the de-interleaver, converts the code symbol metrics, $\left\{\underline{\psi}^{(s)}\right\}$, into bit metrics, interleaves the bit metrics using the same interleaver mapping as the corresponding transmitter $s$, and finally converts the interleaved bit metrics into symbol metrics, $\left\{\underline{\phi}^{(s)}\right\}$, which are passed to the soft symbol generator. 


\subsubsection{Soft symbol generator}

The soft symbol generator calculates the average, $\left\{\bar{v}_{n}^{(s)}=E\left[v_{n}^{(s)}\right]\right\}$, of all possible transmitted symbols by transmitter $s$ at time $n$ according to

$$
\bar{v}_{n}^{(s)}=\sum_{d=0}^{M-1} \operatorname{SM}[d] \operatorname{Pr}\left\{d_{n}^{(s)}=d\right\}=\sum_{d=0}^{M-1} \operatorname{SM}[d] \phi_{n}^{(s)}(d)
$$

where SM [.] is the symbol mapping discussed in Section 2.1.3, and $\phi_{n}^{(s)}(d)$ is the a priori probability of symbol $d$ obtained from the MAP decoder after feedback interleaving. The output of the soft symbol generator, $\left\{\underline{\underline{v}}^{(s)}\right\}$, is then passed to the equivalent MIMO channel after applying it to a DFT producing frequency domain soft symbols, $\left\{\bar{V}^{(s)}\right\}$, for transmitter $s$.

\subsubsection{Equivalent MIMO channel}

The equivalent MIMO channel combines the frequency domain soft symbols from all $N_{s}$ soft symbol generators to generate a replica of the interference for each receive antenna. Therefore, the output of the equivalent MIMO channel is the $N_{A}$ feedback signals $\bar{R}_{k}^{(a)}$, for all $a \in\left\{1,2, \cdots, N_{A}\right\}$ and $k \in\{0,1, \cdots, N-1\}$, where $\bar{R}_{k}^{(a)}$ is given by

$$
\bar{R}_{k}^{(a)}=\sum_{s=1}^{N_{s}} H_{k}^{(s, a)} E\left[V_{k}^{(a)}\right]+E\left[W_{k}^{(a)}\right]
$$

and since the AWGN have zero mean $\left(E\left[W_{k}^{(a)}\right]=0\right)$, therefore

$$
\bar{R}_{k}^{(a)}=\sum_{s=1}^{N_{s}} H_{k}^{(s, a)} \bar{V}_{k}^{(s)}
$$

where $H_{k}^{(s, a)}$ is the DFT of the discrete-time channel impulse response between the $s^{t h}$ transmitter and the $a^{t h}$ receiver antenna, which is estimated by the MU receiver. These $N_{A}$ signals are then fed back to each of the $N_{s}$ detectors as illustrated in Figure 2.5 . 


\subsection{Link-level Simulation Results}

This section presents computer simulation packet error rate (PER) performance results for the MIMO detector with the modified channel model used in this research. These results represent the link-level simulation results that will be used later in the system level simulations. The purpose of these link-level results is to show the effectiveness of the MIMO detector in cancelling MAI for different interferer powers (i.e. different values of the $\Delta$ parameter).

The curves show the PER of the messages received by a $\mathrm{MU}$, having $N_{A}$ receive antennas, from the desired transmitter in the presence of $N_{s}-1$ interferers forming a $\left(N_{A}, N_{s}\right)$ MIMO system as mentioned before. PER performance results are shown for this MIMO system with and without using the IC, denoted (w-IC) and (w/o-IC) respectively. Results are also shown for the case with no MAI which corresponds to a $\left(N_{A}, N_{s}=1\right)$ MIMO system and is denoted (w/o-MAI).

All link-level simulations are based on the following link parameters. Because the radix-2 FFT operation requires the number of symbols in a block to be a power of two, we chose a block size of $N=1024$ symbols for all simulations. The convolutional encoder used by all transmitters has rate $1 / 2$, with generator polynomial of $(5,7)_{8}$, and a constraint length of three $\left(L_{c}=3\right)$. Therefore, the message block size is $N_{a}=$ 1022 message symbols and each message symbol consists of one bit $\left(n_{a}=1\right)$. Pseudorandom bit interleaving is used, with an interleaver size of 2048 bits. The results shown were taken after five iterations of the receiver. The total number of message words simulated for every SNR points is 20000. All simulations were done using the QPSK constellation shown in Figure 2.3. The discrete-time baseband ISI channel follows the SUI-5 channel model [23] which has a power delay profile as shown in 


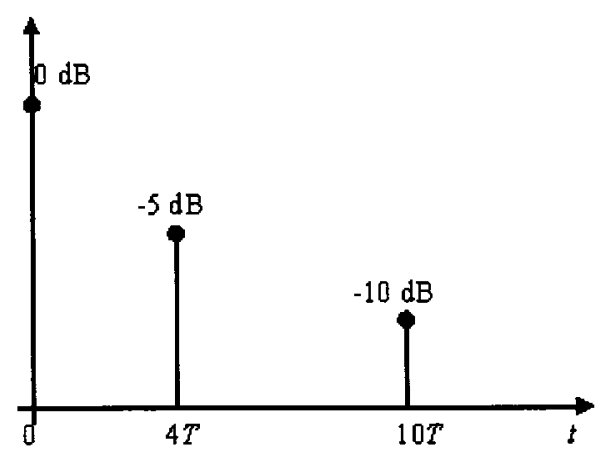

Figure 2.8: Delay spread profile for SUI-5 channel.

Figure 2.8. The SUI-5 channel has three taps with unequal variances (specifically, $0.706,0.223$ and 0.0706 ), and a delay spread covering 11 symbols. The receiver sampling rate is equal to the symbol rate. Finally, simulations were performed for two MIMO systems. The first is $\left(N_{s}=2, N_{A}=1\right)$ for a MU with a single antenna, and a single interferer, where the w/o-MAI system is $\left(N_{s}=1, N_{A}=1\right)$. And the second is $\left(N_{s}=4, N_{A}=2\right)$ for a $\mathrm{MU}$ with two receive antennas, and three interferers, where the w/o-MAI system is $\left(N_{s}=1, N_{A}=2\right)$.

\subsubsection{Simulation Results for $\left(N_{s}=2, N_{A}=1\right)$}

In this case simulations were done for $\Delta_{1}=0 \mathrm{~dB}$ for the desired transmitter, and $\Delta_{2} \in[-20,20] \mathrm{dB}$ for the interferer. For each value of $\Delta_{2}$ a PER table was created for several SNR points for both (w-IC) and (w/o-IC) cases. Recall that larger $\Delta_{2}$ values indicate weaker interferer power, while smaller $\Delta_{2}$ values indicate stronger interferer power. Also, it is good here to emphasize that in this case all the improvement for the (w-IC) system over the (w/o-IC) system is achieved merely through signal processing at the receiver with a single receive antenna.

Figures 2.9 and 2.10 show PER performance results for selected values of $\Delta_{2}$ for 
(w-IC) and (w/o-IC) respectively. The effect of the IC can be seen by the removal of the error floors seen in Figure 2.10 for the (w/o-IC) case. These error floors occur at higher PERs with the increase of the interferer power or equivalently the decrease of $\Delta_{2}$. Moreover, these error floors are better removed for strong interferers than weaker ones as they can be better detected and cancelled. This is illustrated more in Figures 2.11, 2.12 and 2.13 for $\Delta_{2}=0 \mathrm{~dB}$ (interferer with equal power to $\mathrm{MU}$ ), $\Delta_{2}$ $=20 \mathrm{~dB}$ (weak interferer), and $\Delta_{2}=-20 \mathrm{~dB}$ (strong interferer) respectively.

When $\Delta_{2}=0 \mathrm{~dB}$, the interferer has reasonable power and hence the $\mathrm{IC}$ is able to detect and cancel its signal. Note that the IC performance approaches the (w/o-MAI) case with the increase of SNR due to better detection and hence cancellation of the interferer signal while without the IC an error floor is reached at a very high PER close to 1 . For a very weak interferer $\left(\Delta_{2}=20 \mathrm{~dB}\right)$, the IC is unable to detect the interferer and hence (w-IC) and (w/o-IC) have a similar performance. However, the (w-IC) case is expected to approach (w/o-MAI) at higher SNRs, where the interferer can be detected and cancelled, while the (w/o-IC) case will suffer from an error floor which occurs at a lower PER due to the low interferer power. Finally, for a very strong interferer $\left(\Delta_{2}=-20 \mathrm{~dB}\right)$ the IC can very efficiently detect and cancel the interferer signal performing as good as the (w/o-MAI) case while the (w/o-IC) case is totally in error. 


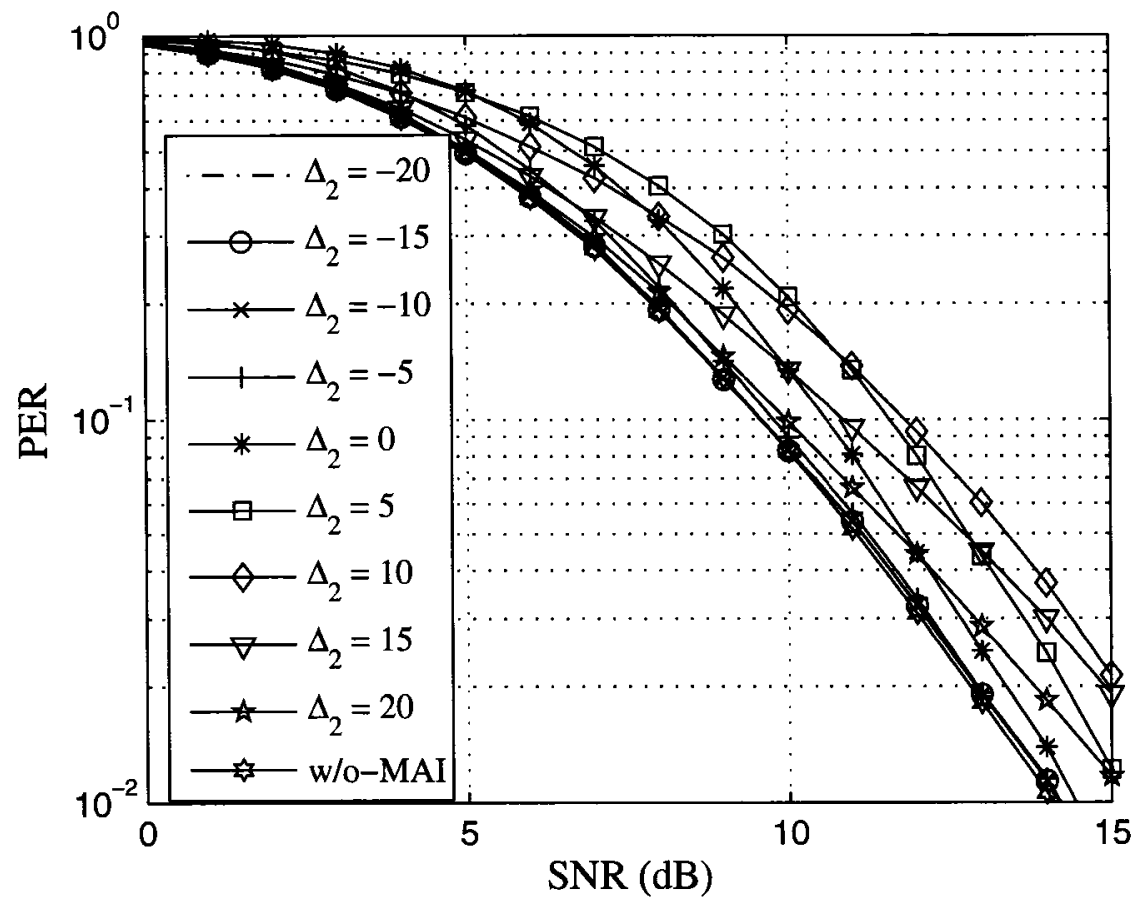

Figure 2.9: MIMO detector (w-IC) PER performance for $\left(N_{s}=2, N_{A}=1\right), \Delta_{1}=0$ $\mathrm{dB}, \Delta_{2} \in[-20,20] \mathrm{dB}$, SUI-5 channel model and QPSK.

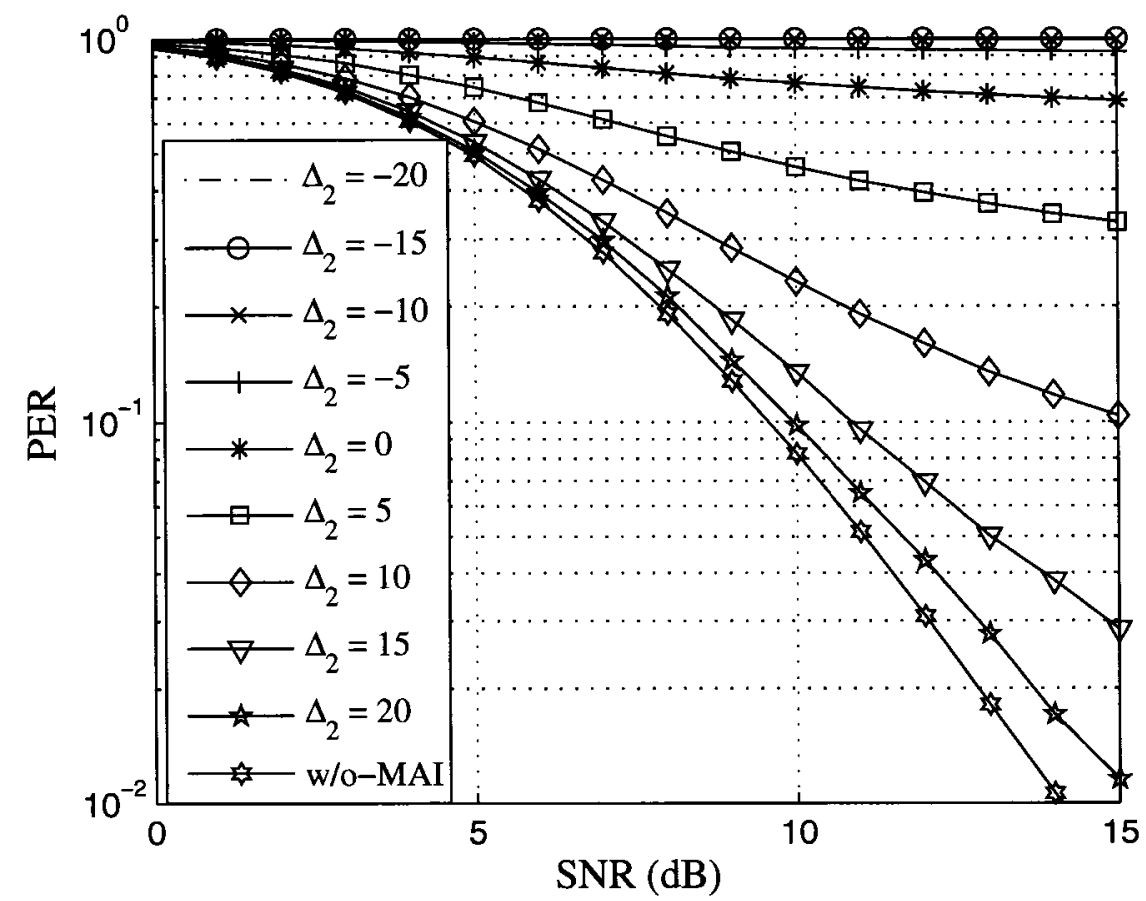

Figure 2.10: MIMO detector (w/o-IC) PER performance for $\left(N_{s}=2, N_{A}=1\right), \Delta_{1}=$ $0 \mathrm{~dB}, \Delta_{2} \in[-20,20] \mathrm{dB}$, SUI-5 channel model and QPSK. 


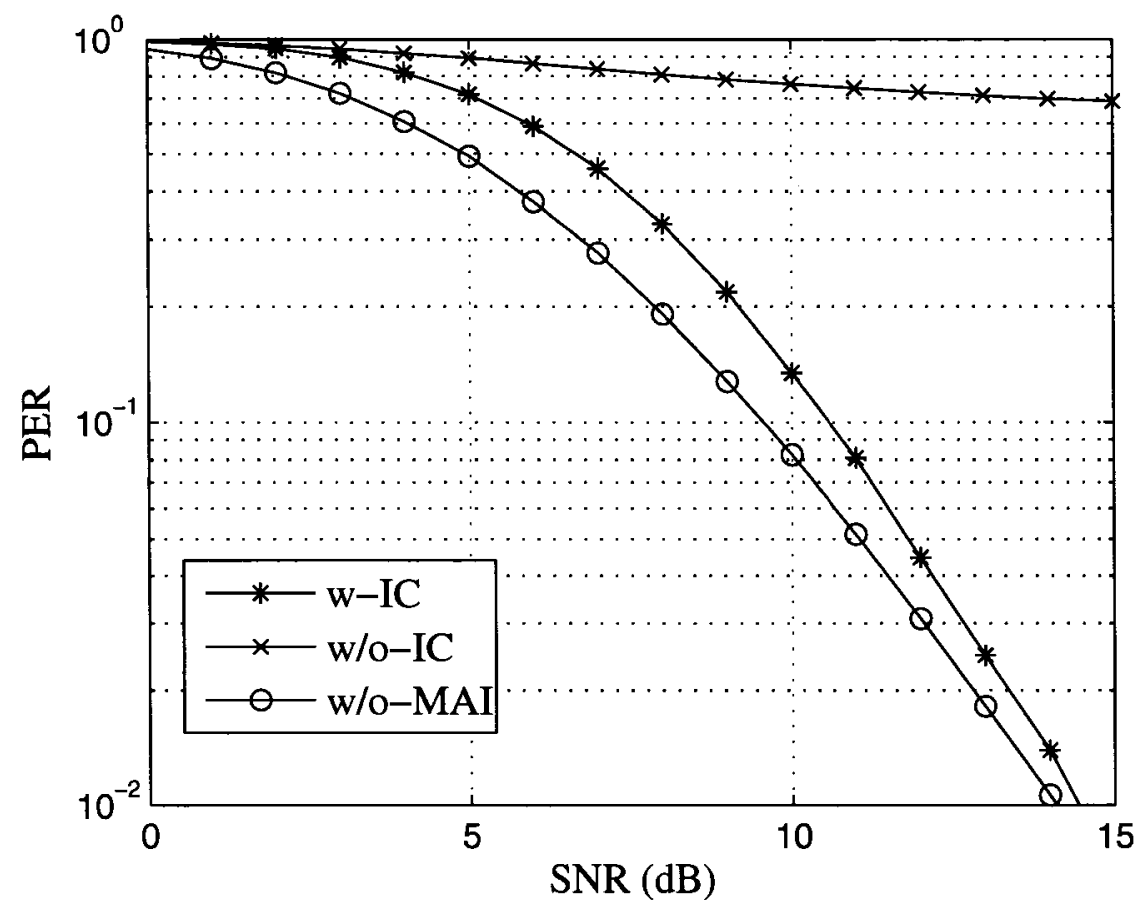

Figure 2.11: MIMO detector (w-IC) PER performance for $\left(N_{s}=2, N_{A}=1\right), \Delta_{1}=0$ $\mathrm{dB}, \Delta_{2}=0 \mathrm{~dB}$, SUI-5 channel model and QPSK.

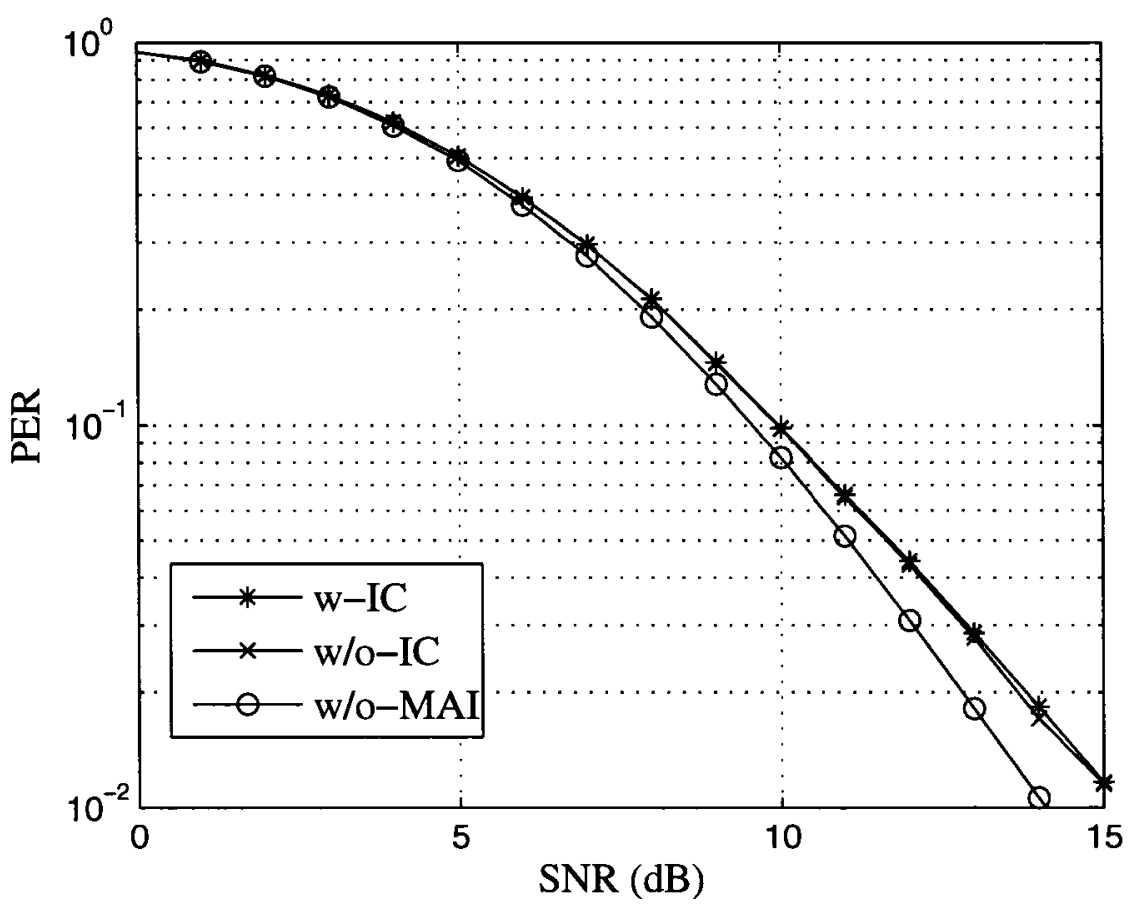

Figure 2.12: MIMO detector (w-IC) PER performance for $\left(N_{s}=2, N_{A}=1\right), \Delta_{1}=0$ $\mathrm{dB}, \Delta_{2}=20 \mathrm{~dB}$, SUI-5 channel model and QPSK. 


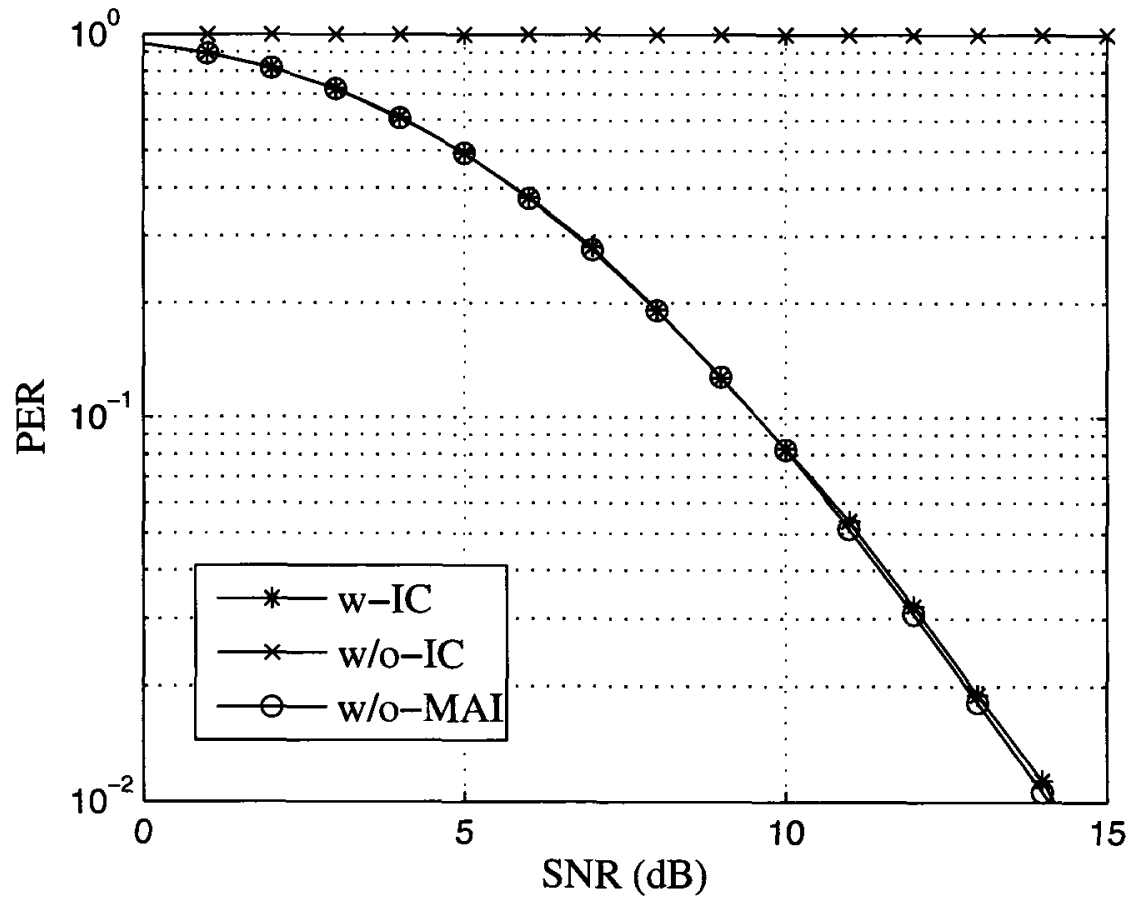

Figure 2.13: MIMO detector (w-IC) PER performance for $\left(N_{s}=2, N_{A}=1\right), \Delta_{1}=0$ $\mathrm{dB}, \Delta_{2}=-20 \mathrm{~dB}$, SUI-5 channel model and QPSK. 


\subsubsection{Simulation Results for $\left(N_{s}=4, N_{A}=2\right)$}

In this case, due to the existence of three interferers, running simulations for all $\left(\Delta_{2}, \Delta_{3}, \Delta_{4}\right)$ combinations with each one of them ranging from $[-20,20] \mathrm{dB}$ would require a prohibitive amount of simulations. For that reason only a few combinations of $\left(\Delta_{2}, \Delta_{3}, \Delta_{4}\right)$, where $\Delta_{1}$ is always equal to zero, were selected and shown here to illustrate the MIMO detector performance for this case. Due to the difficulty of producing link-level tables for this case, no system-level simulations were done for the $\left(N_{s}=4, N_{A}=2\right)$ case. However, a performance bound for this case, PIC3, where three major interferers are perfectly cancelled is presented to give an idea of the improvement range that could be realized if two receive antennas were available at the MU.

Figures 2.14 to 2.20 show the PER performance of the selected $\left(\Delta_{1}, \Delta_{2}, \Delta_{3}, \Delta_{4}\right)$ combinations. Clear advantages can be seen for the IC in improving the performance in most cases as shown in the figures. Again, the performance of the (w-IC) system approaches the no interference (w/o-MAI) case as the interferers signal power increase, while the (w/o-IC) system performs poorly. This is illustrated through the first three $\left(\Delta_{2}, \Delta_{3}, \Delta_{4}\right)$ combinations.

The first combination is for the case when all interferers have equal power to the MU, where the IC performance is good and improves with the increase of SNR while the (w/o-IC) case error floors at a very high PER. The second combination is for the case of three strong interferers, where the IC performance approaches the (w/o-MAI) case while the performance without the IC is even worse than the first case. And finally the third combination corresponds to the case of three weak interferers where the IC is less able to detect and cancel the interfering signals and hence deviates away from the (w/o-MAI) case while the error floor of the (w/o-IC) case is now at a lower 
PER due to less interference. However, the IC removes the error floor at higher SNR while that is not the case for (w/o-IC).

Figures 2.17 to 2.20 represent combinations between these extreme cases, where not all interferers have the same strength. For example in Figure 2.17 all interferers are weaker than the MU but with different levels while the opposite is shown in Figure 2.18, where all interferers are stronger than the MU but also with different levels. Comparing these two figures we see that the IC favours the second case more where the interference is almost perfectly removed. Figures 2.19 and 2.20 show a mixture of strong and weak interferers, where in the first figure there are two weak and one strong interferer, while in the second there are two strong and one weak interferer. Again we see a better performance for the (w-IC) system in the second case, establishing the fact that the IC benefits from strong interference that can be efficiently detected and cancelled. 


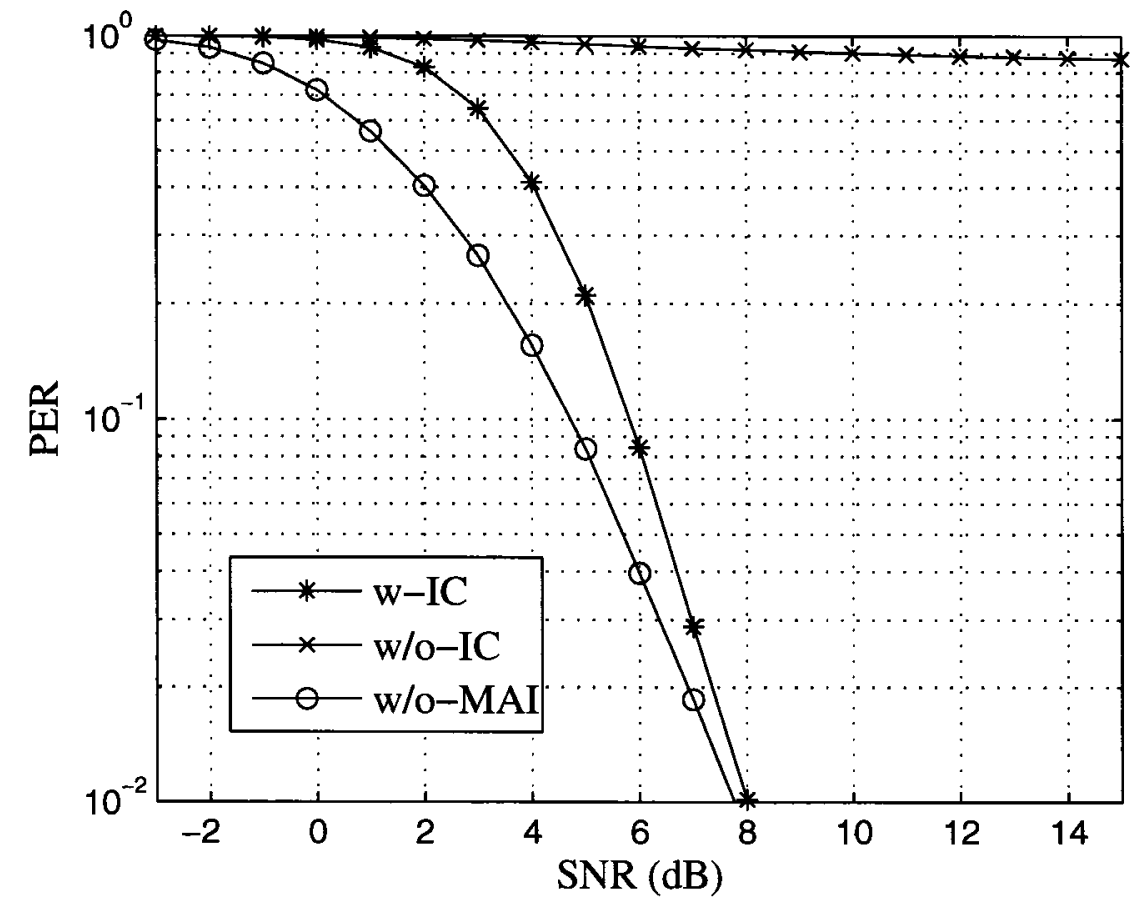

Figure 2.14: MIMO detector PER performance for $\left(N_{s}=4, N_{A}=2\right), \Delta_{1}=0 \mathrm{~dB}, \Delta_{2}$ $=0 \mathrm{~dB}, \Delta_{3}=0 \mathrm{~dB}, \Delta_{4}=0 \mathrm{~dB}$, SUI-5 channel model, and QPSK.

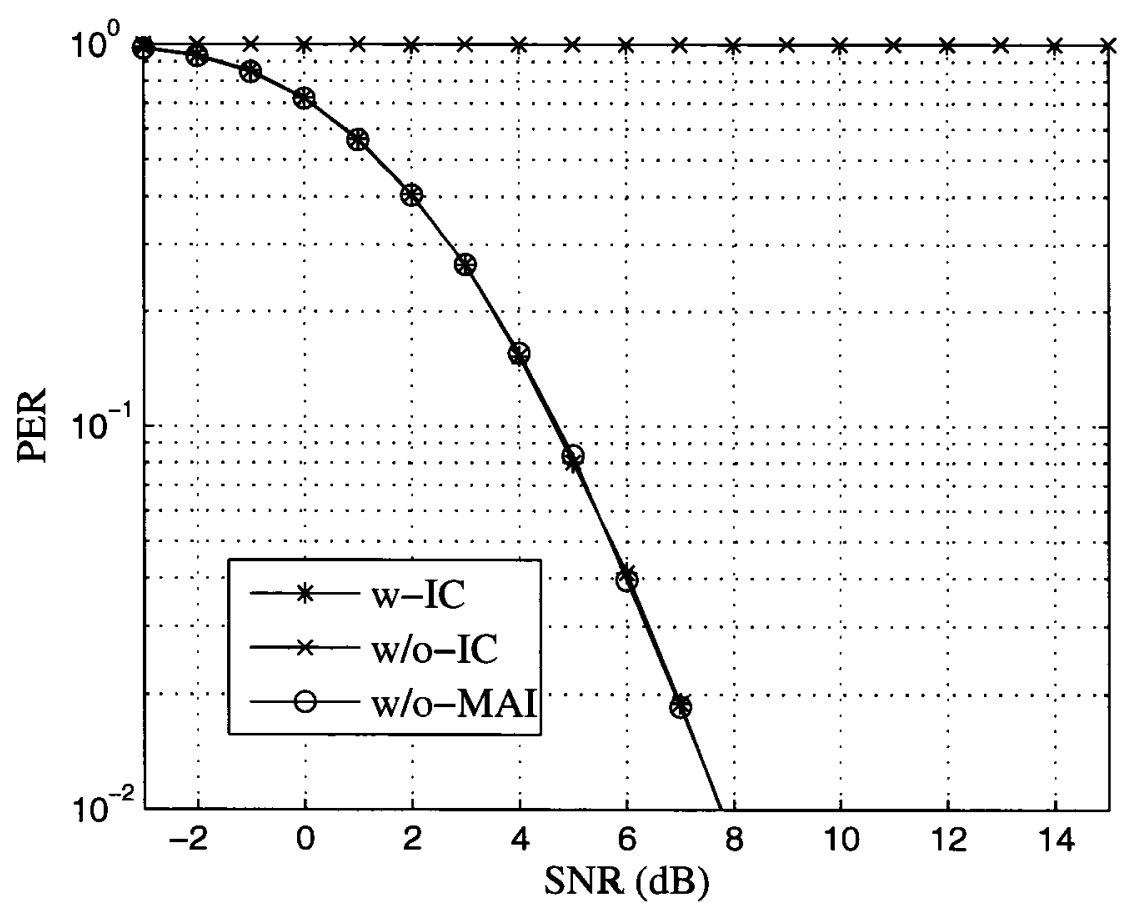

Figure 2.15: MIMO detector PER performance for $\left(N_{s}=4, N_{A}=2\right), \Delta_{1}=0 \mathrm{~dB}, \Delta_{2}$ $=-10 \mathrm{~dB}, \Delta_{3}=-10 \mathrm{~dB}, \Delta_{4}=-10 \mathrm{~dB}$, SUI-5 channel model, and QPSK. 


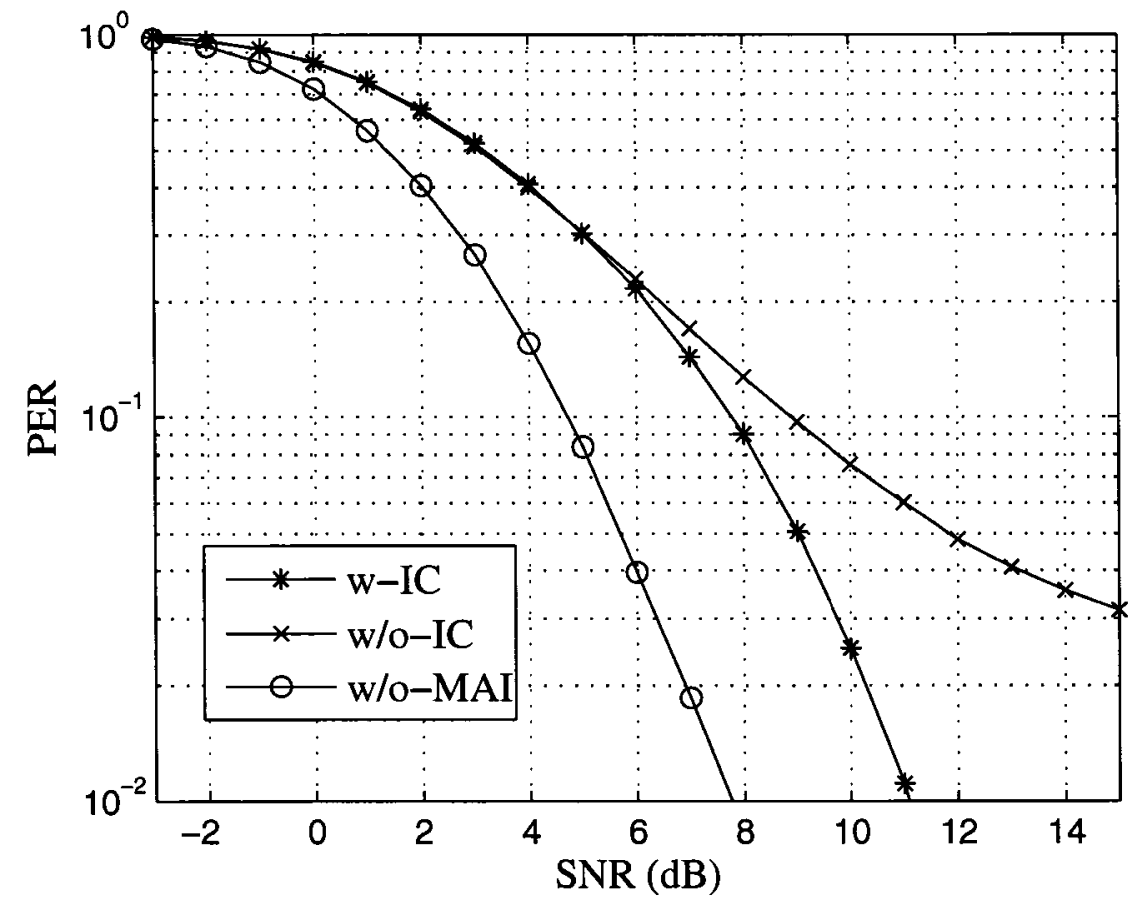

Figure 2.16: MIMO detector PER performance for $\left(N_{s}=4, N_{A}=2\right), \Delta_{1}=0 \mathrm{~dB}, \Delta_{2}$ $=10 \mathrm{~dB}, \Delta_{3}=10 \mathrm{~dB}, \Delta_{4}=10 \mathrm{~dB}$, SUI-5 channel model, and QPSK.

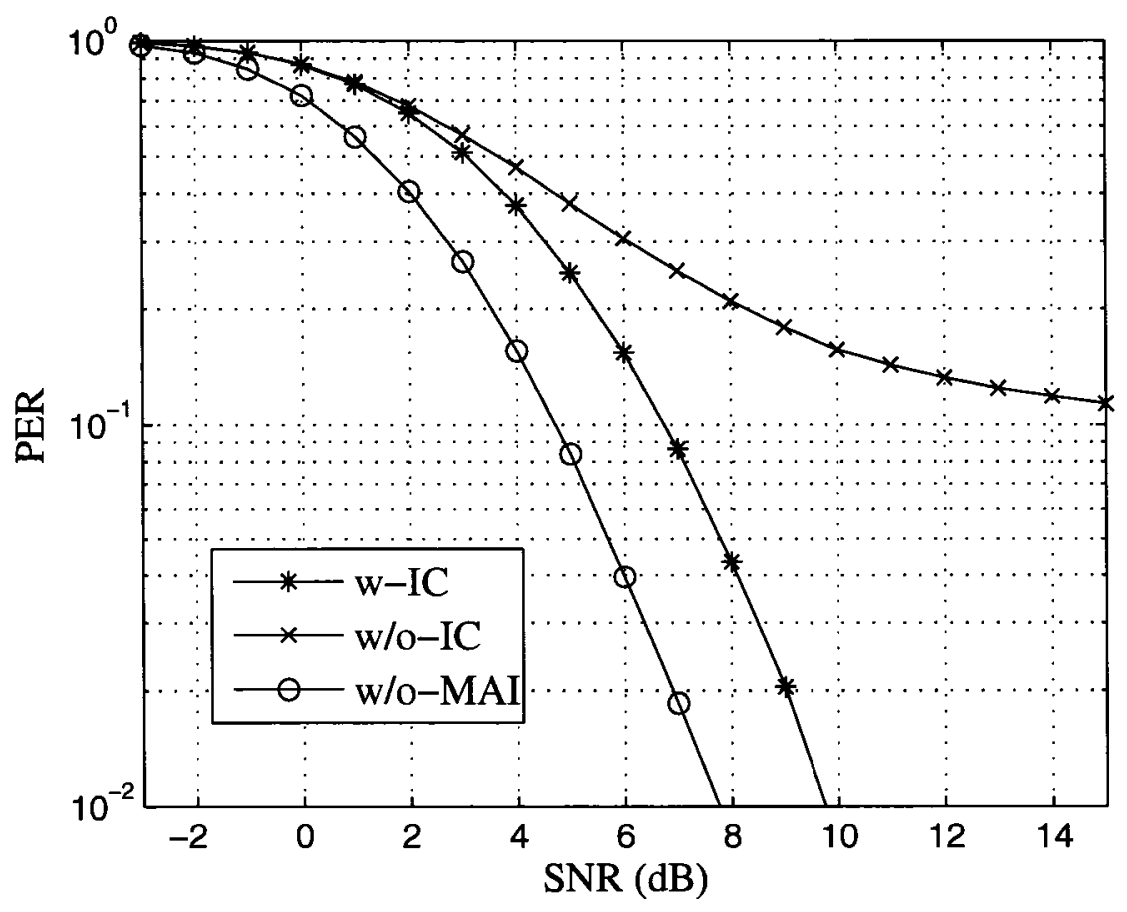

Figure 2.17: MIMO detector PER performance for $\left(N_{s}=4, N_{A}=2\right), \Delta_{1}=0 \mathrm{~dB}, \Delta_{2}$ $=5 \mathrm{~dB}, \Delta_{3}=10 \mathrm{~dB}, \Delta_{4}=15 \mathrm{~dB}$, SUI-5 channel model, and QPSK. 


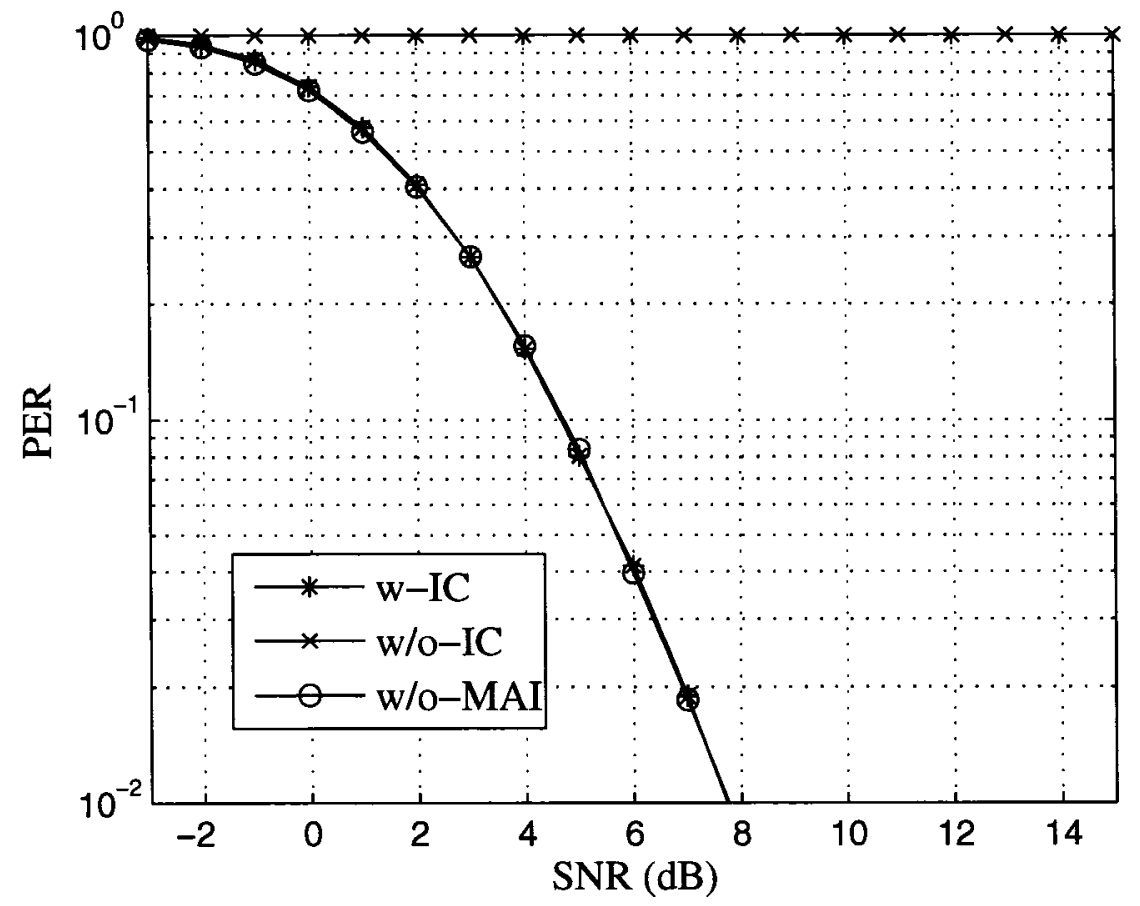

Figure 2.18: MIMO detector PER performance for $\left(N_{s}=4, N_{A}=2\right), \Delta_{1}=0 \mathrm{~dB}, \Delta_{2}$ $=-5 \mathrm{~dB}, \Delta_{3}=-10 \mathrm{~dB}, \Delta_{4}=-15 \mathrm{~dB}$, SUI-5 channel model, and QPSK.

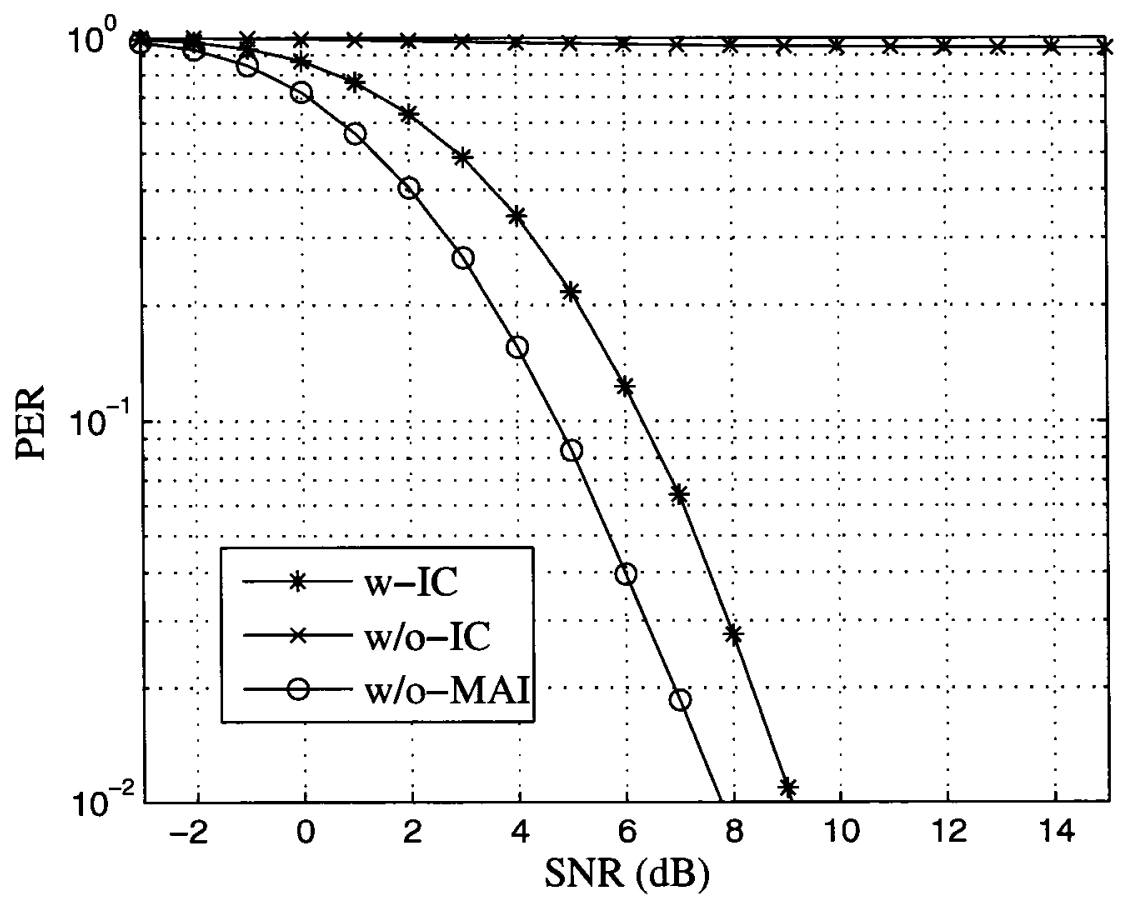

Figure 2.19: MIMO detector PER performance for $\left(N_{s}=4, N_{A}=2\right), \Delta_{1}=0 \mathrm{~dB}, \Delta_{2}$ $=-5 \mathrm{~dB}, \Delta_{3}=5 \mathrm{~dB}, \Delta_{4}=10 \mathrm{~dB}$, SUI-5 channel model, and QPSK. 


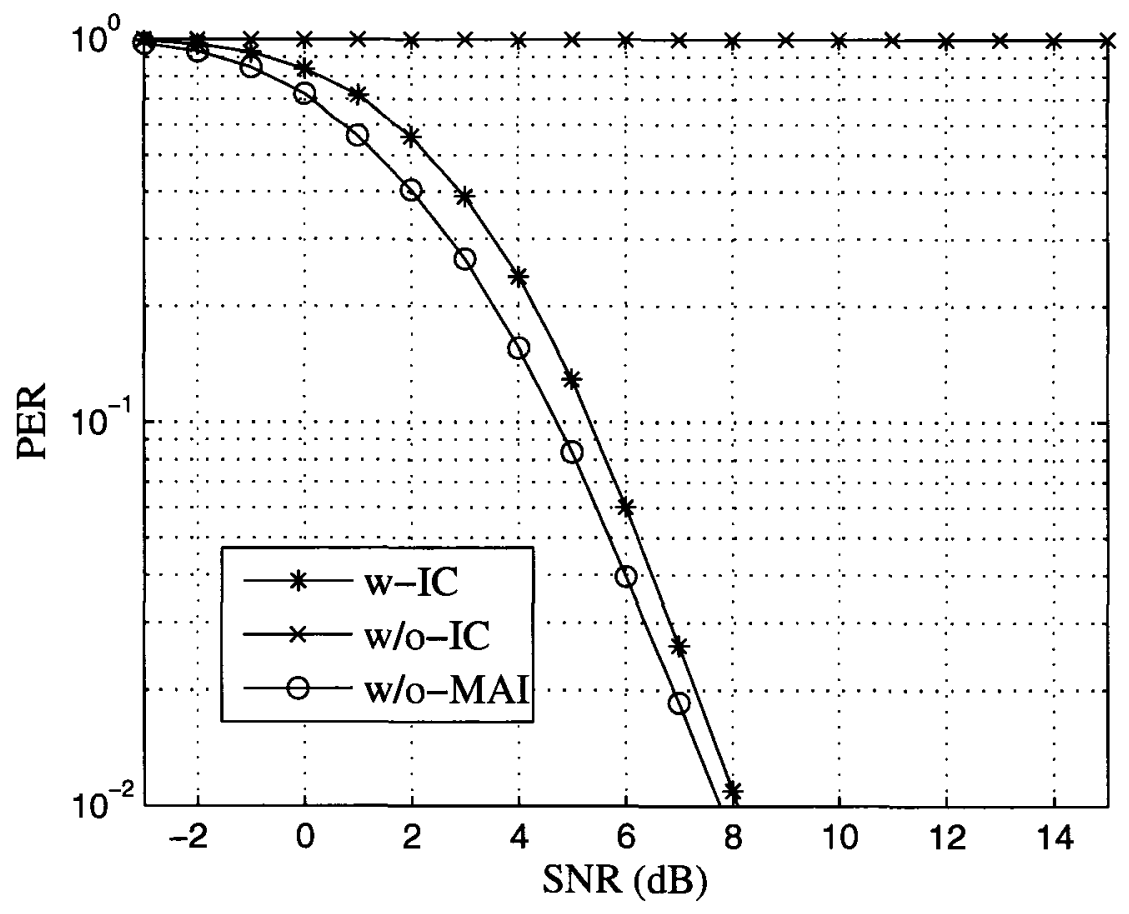

Figure 2.20: MIMO detector PER performance for $\left(N_{s}=4, N_{A}=2\right), \Delta_{1}=0 \mathrm{~dB}, \Delta_{2}$ $=5 \mathrm{~dB}, \Delta_{3}=-5 \mathrm{~dB}, \Delta_{4}=-10 \mathrm{~dB}$, SUI-5 channel model, and QPSK. 


\section{Chapter 3}

\section{Simulation Model}

This chapter outlines the system model used in this research. First, aspects concerning the wireless channel propagation model, calculation of the signal-tointerference-and-noise ratio (SINR), and the antenna patterns used, are discussed. After that the cellular layout used in this research as well as the relaying protocols employed in multihop networks are described. Finally the environment parameters and assumptions used in the computer simulations as well as the simulation algorithm are presented.

\subsection{The Wireless Channel Propagation Model}

The wireless channel is the physical transmission medium over which the information is transmitted. It is characterized by several physical limitations that distort, attenuate, and add noise to the transmitted signal. These physical limitations are reflected in the propagation model used which is usually derived using a combination of analytical and empirical methods.

Propagation models can be classified into large-scale and small-scale models. Large-scale models predict the mean signal strength for an arbitrary transmitterreceiver (T-R) separation distance. Small-scale models on the other hand characterize the rapid fluctuations of the received signal strength over very short travel distances 
or short time durations $[2,24]$.

\subsubsection{Large-Scale Propagation Model}

According to both theoretical and measurement-based propagation models the average received signal power decreases logarithmically with distance. The average large-scale path loss for an arbitrary T-R separation is expressed as a function of distance using a path loss exponent as follows

$$
\bar{L}_{P}(d)=\bar{L}_{P}\left(d_{0}\right)+10 \rho \log \left(\frac{d}{d_{0}}\right)
$$

where $\rho$ is the path loss exponent that indicates the rate at which the path loss increases with distance, $d_{0}$ is the close-in reference distance which is determined from measurements close to the transmitter, and $d$ is the T-R separation. The bars in the equation indicates the ensemble average of all possible path loss values for a given value of $d$. The path loss at the close-in reference distance $\bar{L}_{P}\left(d_{0}\right)$ is calculated according to the free space path loss formula given by

$$
\bar{L}_{P}\left(d_{0}\right)=-10 \log \left[\frac{\lambda^{2}}{(4 \pi)^{2} d_{0}^{2}}\right]
$$

where $\lambda$ is the wavelength of the carrier.

The measured path loss at two different locations having the same T-R separation may be vastly different from the average value predicted by (3.1). In particular, measurements have shown that at any value of $d$, the path loss $L_{P}(d)$ at a particular location is random and distributed log normally (normal in dB) about the mean distance-dependent value calculated from (3.1). That is,

$$
L_{P}(d)=\bar{L}_{P}(d)+L_{S}=\bar{L}_{P}\left(d_{0}\right)+10 \rho \log \left(\frac{d}{d_{0}}\right)+L_{S}
$$


where $L_{S}$ is a zero-mean Gaussian distributed random variable (in $\mathrm{dB}$ ) with standard deviation $\sigma_{S}$ (also in $\mathrm{dB}$ ). This log-normal random variable represents what is known as log-normal shadowing, which occurs over a large number of measurement locations which have the same T-R separation but have different levels of clutter on their propagation paths.

\subsubsection{Small-Scale Propagation Model}

Small-scale fading reflects the rapid fluctuations of the amplitude, phases, or multipath delays of a radio signal over a short period of time or travel distance. This fluctuation occurs due to the vectorial addition at the receiver antenna of multipath components of the signal arriving from different directions and with different propagation delays. This fluctuation occurs even if the receiver is stationary due to the movement of surrounding objects in the radio channel.

Fading is characterized by two important parameters, which are the coherence bandwidth, $B_{c}$, and the coherence time, $T_{c}$. $B_{c}$ is the range of frequencies over which all spectral components have approximately equal gain and linear phase (i.e., flat), while $T_{c}$ is the time duration over which the channel impulse response is essentially invariant. Fading is said to be flat if the signal bandwidth is less than the coherence bandwidth, otherwise it is said to be frequency selective. Similarly, fading is considered to be slow if the coherence time is greater than the symbol period and it is considered to be fast otherwise.

In mobile radio channels, the Rayleigh distribution is commonly used to describe the statistical time-varying nature of the received envelope of a flat fading signal, or the envelope of an individual multipath component. When there is a dominant stationary (non-fading) signal component, such as a line-of-sight propagation path, 
the fading distribution is Ricean. As the dominant signal becomes weaker, the Ricean distribution degenerates to a Rayleigh distribution [11].

\subsection{SINR Calculation}

To determine the performance of a randomly located MU from the link-level simulation results presented in Section 2.6, the signal-to-interference-and-noise-ratio (SINR) needs to be calculated. The SINR for a $\mathrm{MU}$ is the ratio between the received signal power $\left(P_{r}\right)$ and the summation of both the total interference power $\left(P_{I}\right)$ and the noise power $\left(P_{N}\right)$. The MU received signal power in the downlink direction is calculated from the link budget equation given by [2]

$$
P_{r}=P_{t}+G_{t}(\theta)+G_{r}-\bar{L}_{P}(d)-L_{S}
$$

where

$$
\begin{aligned}
& P_{r}=\text { received signal power at the MU receiver } \\
& P_{t}=\text { transmitted signal power } \\
& G_{t}(\theta)=\text { transmitting antenna gain, for transmit angle } \theta \\
& G_{r}=\text { receiving antenna gain } \\
& \bar{L}_{P}=\text { path loss as given by }(3.1) \\
& L_{S}=\text { log normal shadowing in } \mathrm{dB}
\end{aligned}
$$

The received power from the desired transmitter constitutes the desired signal power $\left(P_{S}\right)$, while the received power from interfering units is added to the total interference power $\left(P_{I}\right)$. The link budget equation takes into account the effects of antenna gains, distance dependent path loss, and shadowing. The effect of fading can be included in the link budget as well or may equivalently be averaged out in the 
link-level simulations which is the approach adopted in this research. The antenna patterns used in this research is investigated in the next section.

Having defined how $P_{S}$ and $P_{I}$ are calculated the SINR can be calculated according to

$$
\mathrm{SINR}=\frac{P_{S}}{P_{I}+P_{N}}
$$

where

$$
\begin{aligned}
& P_{S}=\text { MU received desired signal power } \\
& P_{I}=\text { total interference power from all other users } \\
& P_{N}=\text { total channel noise power }
\end{aligned}
$$

The total channel noise power is comprised of both thermal noise, $N_{T}$, and the noise figure, $N_{F}$, as given by (3.6). The noise figure relates the signal-to-noise-ratio (SNR) at the input of the receiver to the SNR at its output thus measuring the SNR degradation caused by the receiver [25], and

$$
P_{N}=N_{T}+N_{F}
$$

The thermal noise in $\mathrm{dBm}$ is given by

$$
N_{T}=10 \log \left(\frac{K_{b} \times T_{K} \times W}{1 \times 10^{-3}}\right)
$$

where $K_{b}=1.38 \times 10^{-23}$ Joules/Kelvin is Boltzmann's constant, $T_{K}$ is the system temperature in Kelvin, and $W$ is the transmission bandwidth in $\mathrm{Hz}$. The noise figure is taken to be $N_{F}=5 \mathrm{~dB}$ in this work.

\subsection{Antenna Pattern}

The antenna pattern describes the transmitter antenna gain, $G_{t}(\theta)$, as seen by a receiver in a certain direction. In this research an omnidirectional antenna pattern is 


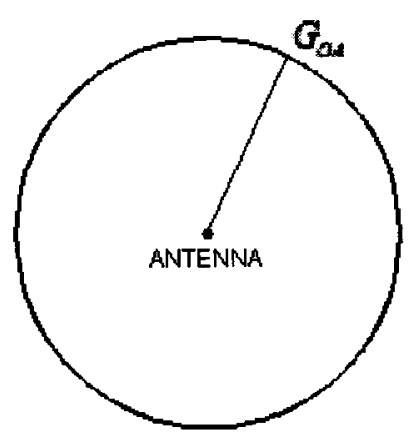

(a)

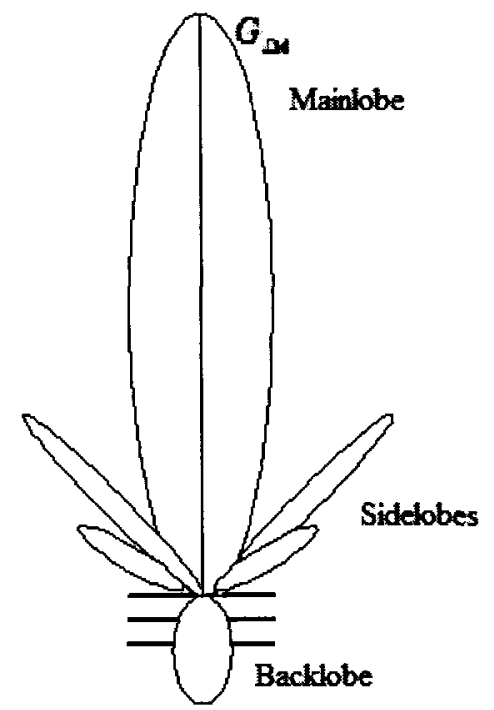

(b)

Figure 3.1: (a) Omnidirectional antenna pattern, (b) Directional antenna pattern.

used for single sector cells while a directional antenna pattern is used for three-sector cells. Omnidirectional antennas have equal antenna gain $\left(G_{\mathrm{OA}}\right)$ in all directions while directional antennas focus the transmitted power in a certain direction (mainlobe) which will have the biggest gain $\left(G_{\mathrm{DA}}\right)$ as shown in Figure 3.1. For a fair comparison between single and three-sectored cells we require that on the average the BS transmit antenna gain experienced by a MU be the same for both cases. Subsection 3.3.1 describes the azimuth antenna pattern of the directional antenna while Subsection 3.3.2 describes how $G_{\mathrm{OA}}$ is derived from a certain $G_{\mathrm{DA}}$ value so that on the average the BS transmit antenna gain as seen by a MU is the same. 


\subsubsection{Directional Antenna Pattern}

The BS antenna pattern for each base station sector (BSS) on the forward link in a sectored cell in this research is given by

$$
G_{t}(\theta)=G_{\mathrm{DA}}-\min \left[12\left(\frac{\theta}{\theta_{3 \mathrm{~dB}}}\right)^{2}, A_{\max }\right]
$$

where

$$
\begin{aligned}
G_{\mathrm{DA}} & =\text { mainlobe antenna gain in } \mathrm{dB} \\
\theta & =\text { horizontal transmit angle at } \mathrm{BS},-180^{\circ} \leq \theta \leq 180^{\circ} \\
\theta_{3 \mathrm{~dB}} & =3 \mathrm{~dB} \text { beamwidth } \\
A_{\max } & =\text { maximum attenuation in } \mathrm{dB}
\end{aligned}
$$

The second term in (3.8) represents the antenna gain attenuation experienced by a user away from the boresight of the antenna by a horizontal transmit angle $\theta$. Figure 3.2 shows a plot of the antenna patten for $G_{\mathrm{DA}}=14 \mathrm{~dB}, \theta_{3 \mathrm{~dB}}=70^{\circ}$, and $A_{\max }=20 \mathrm{~dB}$.

\subsubsection{Omnidirectional Gain Calculation}

In this section the omnidirectional antenna gain $\left(G_{\mathrm{OA}}\right)$ of the $\mathrm{BS}$ in single sector cells which is equal in all directions is derived given a certain directional antenna boresight antenna gain $\left(G_{\mathrm{DA}}\right)$ value. This is done so that on average a mobile unit in a sectored cell gets the same BS transmit antenna gain as another mobile unit in a single sector cell.

Considering a single BSS of a three-sector cell, mobile units will have BS transmit angles $(\theta)$ in the range $\left[-60^{\circ}, 60^{\circ}\right]$. Therefore, they will have a certain range of BS transmit antenna gains $\left(G_{t}\right)$ found by evaluating (3.8) for the previously mentioned 


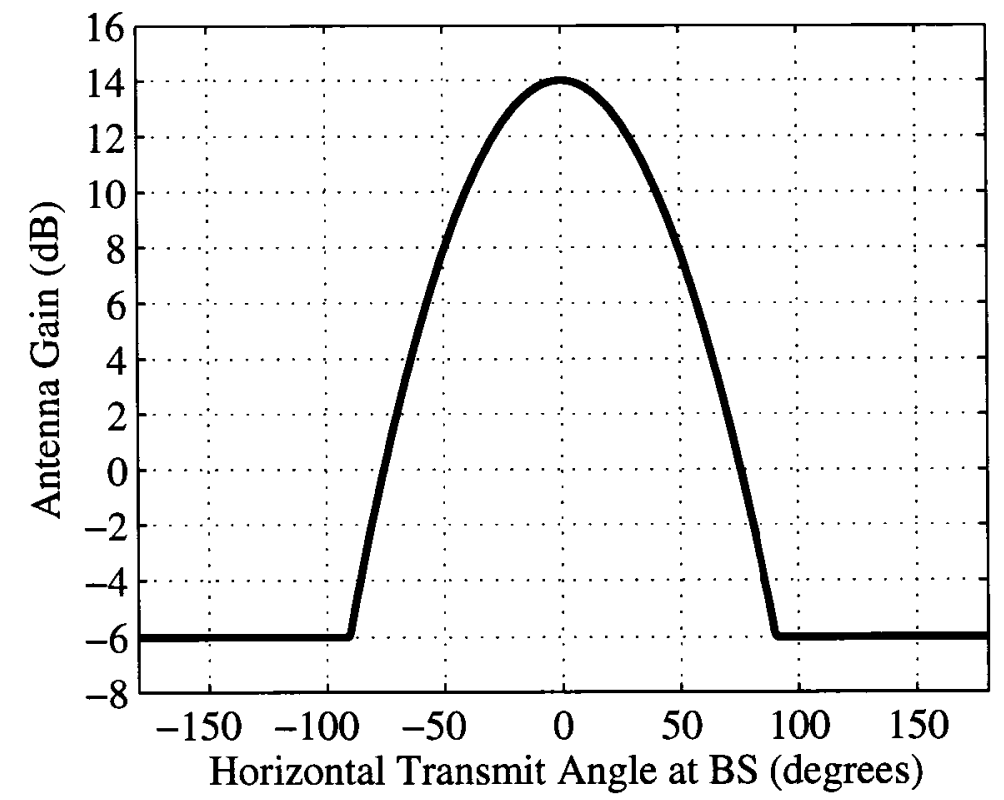

Figure 3.2: BS antenna pattern for each sector, for $G_{\mathrm{DA}}=14 \mathrm{~dB}, \theta_{3 \mathrm{~dB}}=70^{\circ}$, and $A_{\max }=20 \mathrm{~dB}$.

range of $\theta$. In this range the $12\left(\frac{\theta}{\theta_{3 \mathrm{~dB}}}\right)^{2}$ term is always less than $A_{\max }$ as can be seen from Figure 3.2. To calculate $G_{\mathrm{OA}}$ the following steps have to be taken:

1. Find the linear domain equivalent of the directional antenna pattern as

$$
\tilde{G}_{t}(\theta)=10^{G_{t}(\theta) / 10}=10^{\left[G_{\mathrm{DA}}-12\left(\theta / \theta_{3 \mathrm{~dB}}\right)^{2}\right] / 10}
$$


2. Find the average value of $\tilde{G}_{t}(\theta)$ as

$$
\begin{aligned}
\tilde{G}_{\mathrm{OA}} & =\mathrm{E}\left[\tilde{G}_{t}(\theta)\right]=\frac{1}{120} \int_{-60}^{60} \tilde{G}_{t}(\theta) d \theta \\
& =\frac{2}{120} \int_{0}^{60} 10^{G_{\mathrm{DA}} / 10} 10^{-1.2\left(\theta / \theta_{3 \mathrm{~dB}}\right)^{2}} d \theta \\
& =\frac{1}{60} 10^{G_{\mathrm{DA}} / 10} \int_{0}^{60} \exp \left\{-1.2\left(\theta / \theta_{3 \mathrm{~dB}}\right)^{2} \ln 10\right\} d \theta, \text { let } u=\sqrt{1.2 \ln 10}\left(\theta / \theta_{3 \mathrm{~dB}}\right) \\
& =\frac{1}{60} 10^{G_{\mathrm{DA}} / 10} \frac{\sqrt{\pi}}{2} \frac{2}{\sqrt{\pi}} \int_{0}^{\sqrt{1.2 \ln 10} \frac{60}{\theta_{3 \mathrm{~dB}}}} e^{-u^{2}} \frac{\theta_{3 \mathrm{~dB}}}{\sqrt{1.2 \ln 10}} d u \\
& =\frac{1}{60} 10^{G_{\mathrm{DA}} / 10} \frac{\sqrt{\pi}}{2} \frac{\theta_{3 \mathrm{~dB}}}{\sqrt{1.2 \ln 10}} \operatorname{erf}\left(\sqrt{1.2 \ln 10} \frac{60}{\theta_{3 \mathrm{~dB}}}\right)
\end{aligned}
$$

where $\operatorname{erf}(\cdot)$ is the error function.

3. Convert $\tilde{G}_{\mathrm{OA}}$ to $\mathrm{dB}$ as

$$
G_{\mathrm{OA}}=10 \log _{10} \tilde{G}_{\mathrm{OA}}=G_{\mathrm{DA}}+10 \log _{10}\left[\sqrt{\frac{\pi}{1.2 \ln 10}} \frac{\theta_{3 \mathrm{~dB}}}{120} \operatorname{erf}\left(\sqrt{1.2 \ln 10} \frac{60}{\theta_{3 \mathrm{~dB}}}\right)\right]
$$

For a $3 \mathrm{~dB}$ beamwidth of $\theta_{3 \mathrm{~dB}}=70^{\circ}$, this becomes $G_{\mathrm{OA}}=G_{\mathrm{DA}}-2.257$ in $\mathrm{dB}$.

\subsection{Cellular Layouts}

The research in this thesis is based on a hexagonal cellular layout containing seven interfering cells $\left(N_{\mathrm{CELL}}=7\right)$ which may be single $\left(N_{\mathrm{SEC}}=1\right)$ or three $\left(N_{\mathrm{SEC}}=3\right)$ sectored and considering cluster sizes $\left(C_{\mathrm{S}}\right)$ of 1 and 4 as shown in Figure 3.3 for $C_{\mathrm{S}}=1$, and Figure 3.4 for $C_{\mathrm{S}}=4$. We emphasize that for $N_{\mathrm{SEC}}=3$ all three BSSs are using the same channel or frequency band contrary to traditional sectoring where the different BSSs in one cell use different channels. The central BSS in cell \#1 is always the serving BSS while other BSSs act as interferers. So a total of $21 \mathrm{BSSs}$ are modelled for $N_{\mathrm{SEC}}=3$ and $7 \mathrm{BSSs}$ for $N_{\mathrm{SEC}}=1$. For $C_{\mathrm{S}}=4$, the cell sizes 


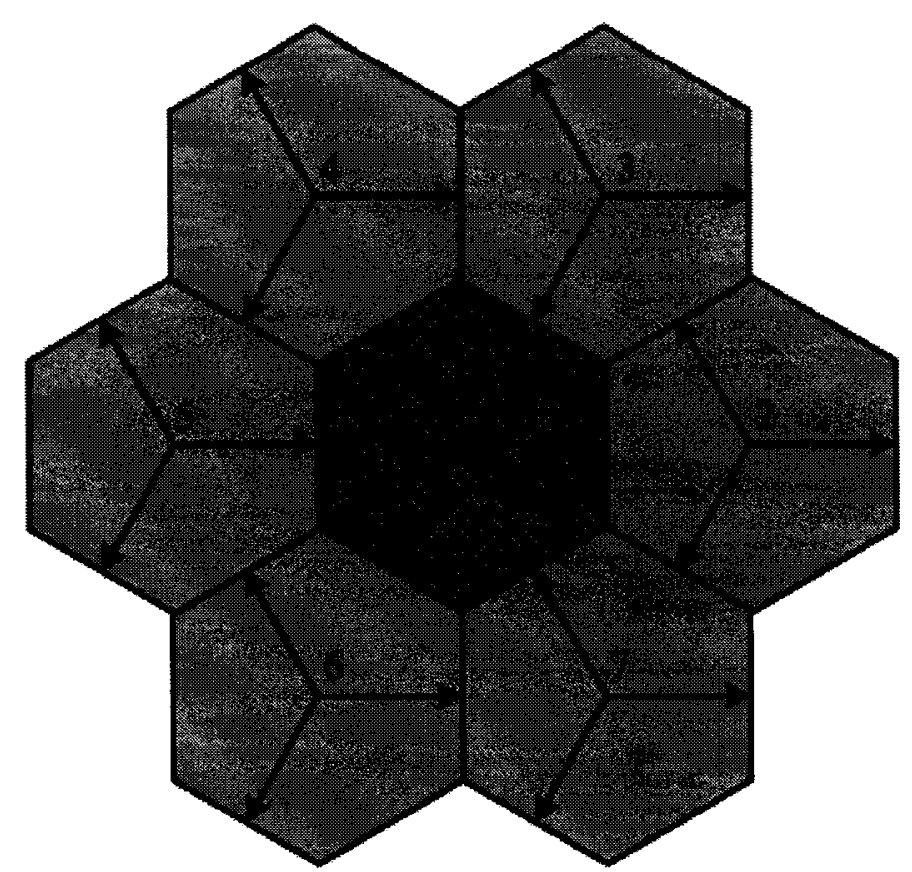

Figure 3.3: Hex cell layout, $C_{\mathrm{S}}=1$, centre cell is in hashed dark grey and interfering cells are in light grey.

remains the same and hence the cells are put further apart from each other. This decreases the CCI from adjacent cells, however CCI from adjacent BSSs in the same cell remain the same. The cell radius is measured as half the distance between the centres of any two adjacent cells.

\subsection{Relaying Protocols}

In this section a hybrid time and frequency domain multiple access (TDMA and FDMA) system is used to describe the relaying protocols, outlining the sources of interference in both the first and second hop. Two different scenarios are considered. In the first scenario, the RSs use different channels than the BSSs. This scenario is denoted as orthogonal relay channel (ORC). In the second scenario, the RSs and 


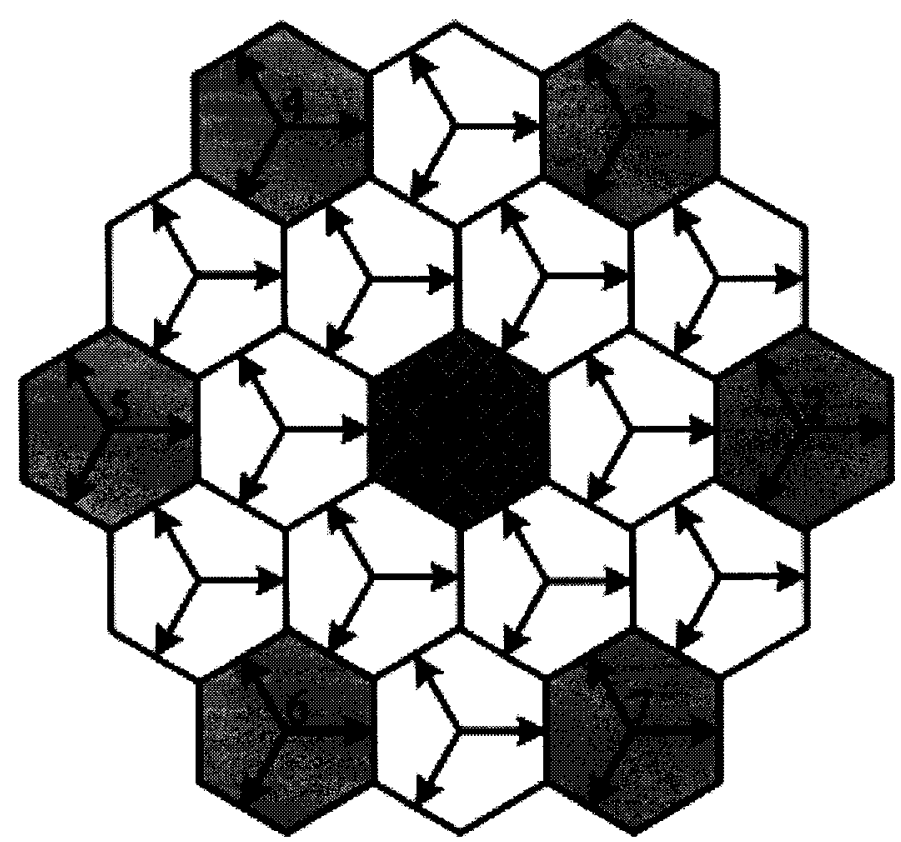

Figure 3.4: Hex cell layout, $C_{\mathrm{S}}=4$, centre cell is in hashed dark grey and interfering cells are in light grey.

BSSs simultaneously use the same channel, denoted as non-orthogonal relay channel (nORC). Thus the ORC case requires an additional channel for relaying, whereas the nORC case does not. A fully loaded system where the BSSs always have traffic to transmit for every time slot is considered. However, for the ORC case the relay channel is used only by as many relays as there are MUs in need of relaying. Although the relaying protocol is the same, the number and sources of interference differ from the ORC case to the $\mathrm{nORC}$ case. After describing each case, the method used to calculate the probability of error as well as the system throughput calculation for both cases is shown. 
Table 3.1: Relaying protocol description, for relaying on ORCs.

\begin{tabular}{|c|c|c|c|}
\hline Hop \# & $\begin{array}{c}\text { Desired } \\
\text { signal }\end{array}$ & Channel & $\begin{array}{c}\text { Sources of } \\
\text { interference }\end{array}$ \\
\hline First & $\mathrm{BSS}_{k} \rightarrow \mathrm{MU}_{k, i}$ & primary & $\left(N_{\mathrm{BSS}}-1\right) \mathrm{BSSs}$ \\
\hline Second & $\mathrm{RS}_{k, i} \rightarrow \mathrm{MU}_{k, i}$ & relay & $\left(0 \rightarrow\left(N_{\mathrm{BSS}}-1\right)\right) \mathrm{RSs}$ \\
\hline
\end{tabular}

\subsubsection{Relaying using ORCs}

Figure 3.5 shows an illustration of the relaying protocol when relays use ORCs for the relaying traffic. In a given time slot, $\mathrm{T}_{n}, \mathrm{BSS}_{k}$ transmits to mobile unit $\mathrm{MU}_{k, i}$, on the primary channel. At the same time all available RSs in $\mathrm{BSS}_{k}$ are also receiving the transmission. This constitutes the first hop transmission where $\mathrm{MU}_{k, i}$ will be receiving interference from all surrounding BSSs that share the same primary channel. In the next time slot, $\mathrm{T}_{n+1}$, while $\mathrm{BSS}_{k}$ is transmitting to another mobile unit $\mathrm{MU}_{k, j}$ on the primary channel, if $\mathrm{MU}_{k, i}$ requests relaying then the $\mathrm{RS}$ with best signal power to $\mathrm{MU}_{k, i}$, which will be denoted $\mathrm{RS}_{k, i}$, retransmits the data to $\mathrm{MU}_{k, i}$ on the relay channel. Therefore, on the second hop, $\mathrm{MU}_{k, i}$ will encounter no interference from any BSS. However, it may receive interference from as many other RSs as the number of MUs in the adjacent BSSs that have requested relaying. So $\mathrm{MU}_{k, i}$ may receive interference from $\mathrm{RS}_{l, h}$ on the second hop such that $k$ and $l$ are not equal. This illustration is summarized in Table 3.1 where $N_{\mathrm{BSS}}$ is the total number of interfering BSSs given by

$$
N_{\mathrm{BSS}}=N_{\mathrm{CELL}} \times N_{\mathrm{SEC}}
$$




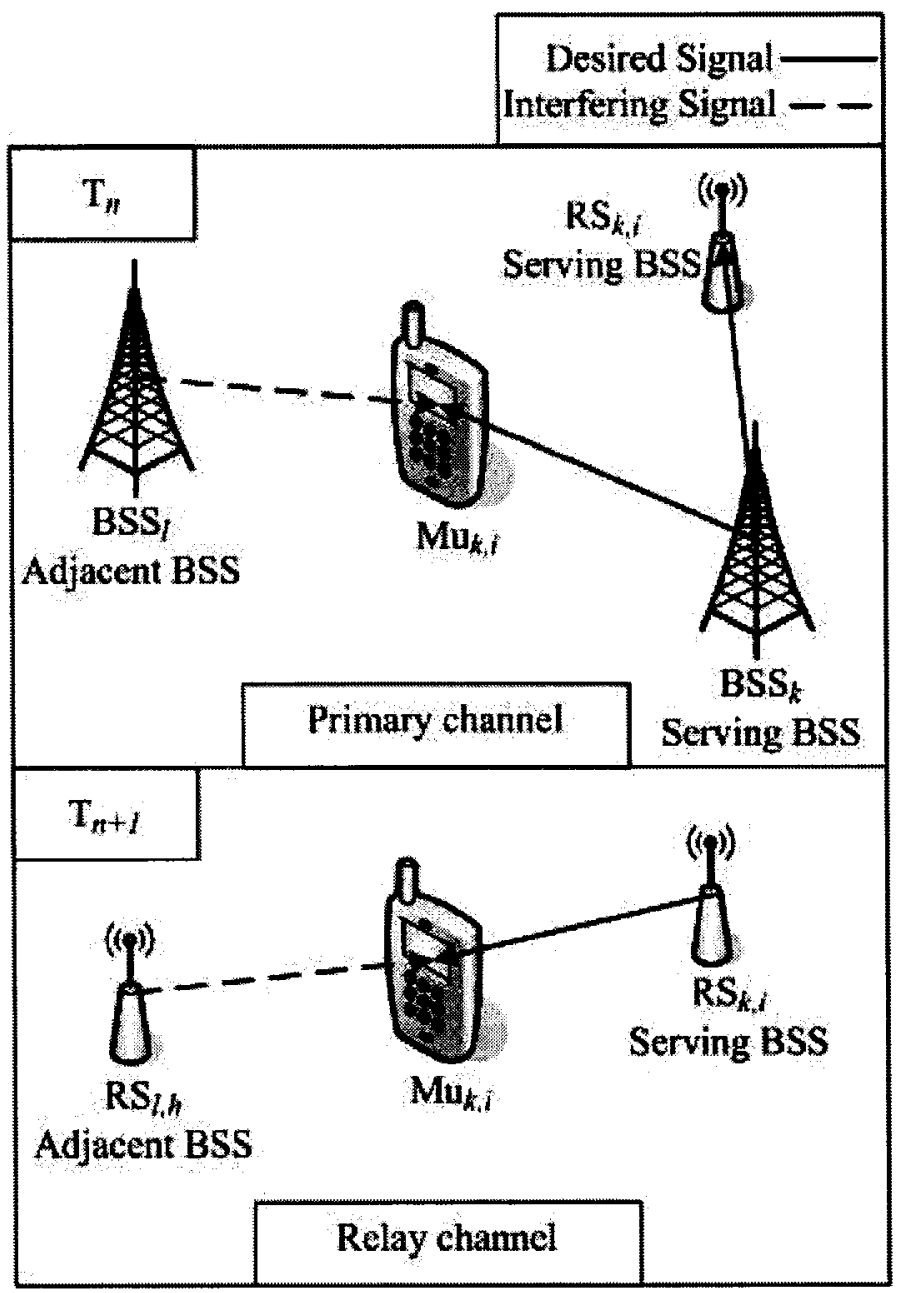

Figure 3.5: Relaying protocol for relaying on orthogonal channels (ORCs).

\subsubsection{Relaying using nORCs}

Figure 3.6 shows an illustration of the relaying protocol when RSs use the same primary channel as BSSs. On the first hop, in addition to interference from adjacent BSSs, a MU may receive interference from any RS that is retransmitting to any other MU that was initially served in the previous time slot, $\mathrm{T}_{n-1}$. These interfering RSs may be in any $\mathrm{BSS}$, including its own $\mathrm{BSS}, \mathrm{BSS}_{k}$. On the second hop, while $\mathrm{RS}_{k, i}$ is transmitting to $\mathrm{MU}_{k, i}$, in addition to the possible interference from any number of 
Table 3.2: Relaying protocol description, for relaying on nORCs.

\begin{tabular}{|c|c|c|c|}
\hline Hop \# & $\begin{array}{c}\text { Desired } \\
\text { signal }\end{array}$ & Channel & $\begin{array}{c}\text { Sources of } \\
\text { interference }\end{array}$ \\
\hline First & $\mathrm{BSS}_{k} \rightarrow \mathrm{MU}_{k, i}$ & primary & $\begin{array}{c}\left(N_{\mathrm{BSS}}-1\right) \mathrm{BSSs}+ \\
\left(0 \rightarrow\left(N_{\mathrm{BSS}}\right)\right) \mathrm{RSs}\end{array}$ \\
\hline Second & $\mathrm{RS}_{k, i} \rightarrow \mathrm{MU}_{k, i}$ & primary & $\begin{array}{c}\left(0 \rightarrow\left(N_{\mathrm{BSS}}-1\right)\right) \mathrm{RSs}+ \\
\left(N_{\mathrm{BSS}}\right) \mathrm{BSSs}\end{array}$ \\
\hline
\end{tabular}

RSs from adjacent sectors there will be interference from all BSSs, including $\mathrm{BSS}_{k}$. This is summarized in Table 3.2 .

\subsubsection{Probability of error calculation}

In this subsection, the method used to calculate the average probability of packet error $\left(\overline{\mathrm{P}}_{e}\right)$ for a $\mathrm{MU}$ is presented. For two-hop systems $\overline{\mathrm{P}}_{e}$ is given by

$$
\overline{\mathrm{P}}_{e}=\overline{\mathrm{P}}_{e, 1} \times \overline{\mathrm{P}}_{e, 2}
$$

where $\overline{\mathrm{P}}_{e, 1}$ and $\overline{\mathrm{P}}_{e, 2}$ are the average packet error rates on the first and second hops respectively. Next, the calculation of $\overline{\mathrm{P}}_{e, 1}$ and $\overline{\mathrm{P}}_{e, 2}$ for both relaying protocols is shown.

\section{Relaying using ORCs}

As shown in Table (3.1) for relaying on ORCs, on the first-hop the BSS-MU transmission experiences interference from a fixed number of neighbour BSSs equal to $N_{\mathrm{BSS}}-1 \mathrm{BSSs}$ while no interference is received from RSs. Therefore, for each MU $\mathrm{P}_{e, 1}$ is equal to the link-level PER for a certain $\operatorname{SNR}(\gamma)$ and $\operatorname{SIR}(\Delta)$ pair. To evaluate $\mathrm{P}_{e, 1}(\gamma, \Delta)$, and based upon the assumption that the different interference 


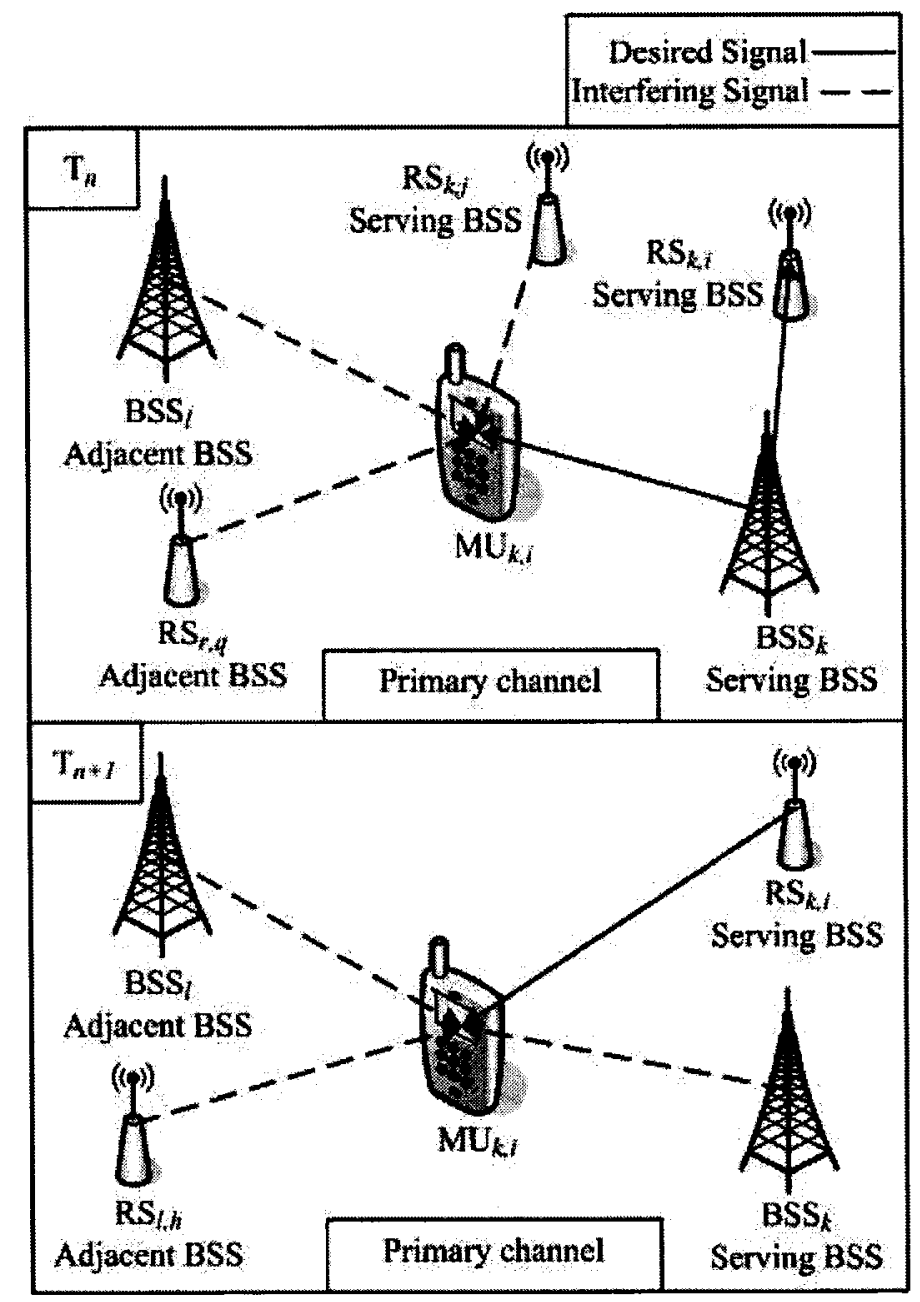

Figure 3.6: Relaying protocol for relaying on non-orthogonal channels (nORCs).

sources are independent from each other, the total interference, $P_{I}$, experienced by the BSS-MU transmission is split in linear domain according to

$$
P_{I}=P_{P}+P_{O}
$$

where $P_{P}$ is the power of the primary interferer and $P_{O}$ is the power of all other interferers. Therefore, in the linear domain $\gamma$ can be calculated according to

$$
\gamma=\frac{P_{S}}{P_{O}+P_{N}}
$$


while $\Delta$ is calculated according to

$$
\Delta=\frac{P_{S}}{P_{P}}
$$

where $P_{O}$ is treated as AWGN and $P_{S}$ and $P_{N}$ are, as defined in (3.5), the signal and noise power respectively. For perfect cancellation of the primary interferer (PIC1), the calculated value of $\gamma$ is used to get the performance from the (w/o-MAI) link-level PER tables. For PIC3, $P_{P}$ in (3.14) is the total power of the three major interferers. $P_{O}$ and $\gamma$ are consequently updated and used to get the PER performance from the w/o-MAI link-level tables. The average probability of error $\overline{\mathrm{P}}_{e, 1}=E\left[\mathrm{P}_{e, 1}(\gamma, \Delta)\right]$ is obtained by averaging over many MUs.

On the second hop, the RS-MU transmission experiences interference from zero to as many as $N_{\mathrm{BSS}}-1$ RSs. Therefore, the number of interfering RSs is random and depends upon the average probability of relaying, which is equivalent to the average probability that the first-hop transmission fails $\left(\overline{\mathrm{P}}_{e, 1}\right)$. Therefore, the instantaneous probability of packet error on the second hop for each $\mathrm{MU}, \mathrm{P}_{e, 2}$, is given by

$$
\mathrm{P}_{e, 2}=\sum_{i=0}^{N_{\mathrm{BSS}}-1}\left(\begin{array}{c}
N_{\mathrm{BSS}}-1 \\
i
\end{array}\right) \operatorname{PER}_{i \mathrm{RSs}}^{(2)}(\gamma, \Delta)\left(1-\overline{\mathrm{P}}_{e, 1}\right)^{\left(N_{\mathrm{BSS}}-1-i\right)} \overline{\mathrm{P}}_{e, 1}^{(i)}
$$

where $\operatorname{PER}_{i \mathrm{RSs}}^{(2)}(\gamma, \Delta)$ is the PER when $i$ RSs are interfering with the RS-MU transmission. These $i$ RSs are used to calculate $P_{P}, P_{O}$, and $P_{I}$ while the signal from the serving RS determines the value of $P_{S}$ where the superscript (2) indicates that the desired signal is from a RS on the second hop. These values are then used to calculate $\gamma$ and $\Delta$ that are consequently used to get the performance results of the different schemes (w/o-IC, w-IC, PIC1, and PIC3) from the appropriate link-level tables. The combinatorial term $\left(\begin{array}{c}N_{\mathrm{BSS}}-1 \\ i\end{array}\right)$ represents the number of ways to choose $i$ RSs from $N_{\mathrm{BSS}}-1 \mathrm{RSs}$, and the $\left(1-\overline{\mathrm{P}}_{e, 1}\right)^{\left(N_{\mathrm{BSS}}-1-i\right)}$ term indicates the success of the first-hop 
transmission for $N_{\mathrm{BSS}}-1-i$ MUs while the term $\overline{\mathrm{P}}_{e, \mathbf{1}}^{(i)}$ indicates first-hop transmission failure for $i$ MUs, and that is why $i$ RSs retransmit and cause interference to the MU in the central BSS.

The average probability of error $\overline{\mathrm{P}}_{e, 2}=\mathrm{E}\left[\mathrm{P}_{e, 2}\right]$ is obtained by averaging over many MUs, giving

$$
\overline{\mathrm{P}}_{e, 2}=\sum_{i=0}^{N_{\mathrm{BSS}}-1}\left(\begin{array}{c}
N_{\mathrm{BSS}}-1 \\
i
\end{array}\right) \overline{\mathrm{PER}}_{i \mathrm{RSs}}^{(2)}\left(1-\overline{\mathrm{P}}_{e, 1}\right)^{\left(N_{\mathrm{BSS}}-1-i\right)} \overline{\mathrm{P}}_{e, 1}^{(i)}
$$

where $\overline{\operatorname{PER}}_{i \mathrm{RSs}}^{(2)}=\mathrm{E}\left[\operatorname{PER}_{i \mathrm{RSs}}^{(2)}(\gamma, \Delta)\right]$ is obtained by averaging over many MUs, and hence $\overline{\mathrm{P}}_{e, 2}$ can be calculated by calculating $\overline{\operatorname{PER}}_{i \mathrm{RSs}}^{(2)}$ for all $i \in\left\{0,1, \cdots, N_{\mathrm{BSS}}-1\right\}$, then applying Equation (3.18). When calculating $\overline{\mathrm{PER}}_{i \mathrm{RSs}}^{(2)}$ through averaging over many MUs, it is important to note that a random combinations of $i$ distinct RSs is chosen for every MU. Finally $\overline{\mathrm{P}}_{e}$ is calculated by substituting $\overline{\mathrm{P}}_{e, 1}$ and $\overline{\mathrm{P}}_{e, 2}$ in (3.13).

\section{Relaying using nORCs}

When relaying is done using nORCs, on the first-hop the BSS-MU transmission may experience interference from RSs in addition to the fixed interference from $N_{\mathrm{BSS}}-$ 1 neighbour BSSs. Therefore, the average packet error rate on the first-hop, $\overline{\mathrm{P}}_{e, 1}$, will be given by

$$
\overline{\mathrm{P}}_{e, 1}=\sum_{i=0}^{N_{\mathrm{BSS}}}\left(\begin{array}{c}
N_{\mathrm{BSS}} \\
i
\end{array}\right) \overline{\mathrm{PER}}_{i \mathrm{RSs}}^{(1)}\left(1-\overline{\mathrm{P}}_{e, 1}\right)^{\left(N_{\mathrm{BSS}}-i\right)} \overline{\mathrm{P}}_{e, 1}^{(i)}
$$

where $\overline{\mathrm{PER}}_{i \mathrm{RSs}}^{(1)}$ is similar to $\overline{\mathrm{PER}}_{i \mathrm{RSs}}^{(2)}$, however the superscript (1) indicates that the desired signal is from a BSS on the first-hop. Also it is important to note that for nORC $\overline{\mathrm{PER}}_{i \mathrm{RSs}}^{(1)}$ does not mean that only $i$ RSs constitute the set of interferers, but it means that $i$ RSs in addition to a fixed number, $N_{\mathrm{BSS}}-1$, of neighbour BSSs form 
that set.

On the second-hop, $\overline{\mathrm{P}}_{e, 2}$ is calculated in the same way as for ORC. Again, it is good to emphasize that the term $\overline{\mathrm{PER}}_{i \mathrm{RSs}}^{(2)}$ is not based upon an interferer set of $i$ RSs

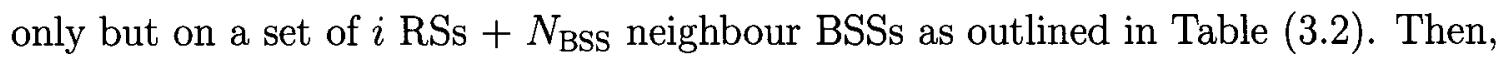
$\overline{\mathrm{P}}_{e}$ is calculated by substituting $\overline{\mathrm{P}}_{e, 1}$ and $\overline{\mathrm{P}}_{e, 2}$ in (3.13).

\subsubsection{System Throughput and Outage Calculation}

In this section, we present the expressions used to calculate the average system throughput $\left(\bar{\eta}_{\mathrm{SYS}}\right)$, the cumulative distribution function (CDF) of the throughput $\left(\eta_{\mathrm{CDF}}\right)$, and the outage probability $\left(\mathrm{P}_{\mathrm{OUT}}\right)$.

First, the MU throughput, $\eta_{\mathrm{MU}}$, measured in bits per channel use (bpchu) is defined as the number of correctly received bits by the MU over a single channel use, and is given by

$$
\eta_{\mathrm{MU}}=\left\{\begin{array}{ccc}
\frac{\varsigma_{\mathrm{MU}}}{2}\left(1-\mathrm{P}_{e}\right) & \text { for } & \mathrm{ORC} \\
\varsigma_{\mathrm{MU}}\left(1-\mathrm{P}_{e}\right) & \text { for } & \mathrm{nORC}
\end{array}\right.
$$

where $\eta_{\mathrm{MU}}$ for ORC is multiplied by a factor of $1 / 2$ due to the use of an orthogonal channel for the relaying traffic, $\mathrm{P}_{e}$ is the $\mathrm{MU}$ probability of packet error over the two hops, and $\varsigma_{\mathrm{MU}}$ is the MU nominal throughput in bpchu which depends upon the constellation size $(M)$ and the code rate $\left(n_{c} / n_{a}\right)$ as follows

$$
\varsigma_{\mathrm{MU}}=\log _{2}(M) \times \frac{n_{c}}{n_{a}}
$$

Next we define the cell throughput, $\eta_{\mathrm{CELL}}$, to be the number of correctly received bits by all MUs in a cell for a single channel use. This throughput definition will be different from $\eta_{\mathrm{MU}}$ for sectored cells, where all sectors use the same band (frequency channel). That is

$$
\eta_{\mathrm{CELL}}=N_{\mathrm{SEC}} \times \eta_{\mathrm{MU}}
$$


The system throughput, $\eta_{\mathrm{SYS}}$, is a scaled version of $\eta_{\text {CELL }}$ which accounts for the cluster size $\left(C_{\mathrm{S}}\right)$ value as follows

$$
\eta_{\mathrm{SYS}}=\frac{1}{C_{\mathrm{S}}} \times \eta_{\mathrm{CELL}}=\frac{N_{\mathrm{SEC}}}{C_{\mathrm{S}}} \times \eta_{\mathrm{MU}}
$$

and hence this can be re-written as

$$
\eta_{\mathrm{SYS}}=\left\{\begin{array}{ccc}
\frac{\mathrm{SSYS}}{2}\left(1-\mathrm{P}_{e}\right) & \text { for } & \text { ORC } \\
\text { ऽSYS }\left(1-\mathrm{P}_{e}\right) & \text { for } & \mathrm{nORC}
\end{array}\right.
$$

where $\varsigma_{S Y S}$ is the system nominal throughput in bits and is given by

$$
\varsigma_{\mathrm{SYS}}=\frac{N_{\mathrm{SEC}} \varsigma_{\mathrm{MU}}}{C_{\mathrm{S}}}=\frac{N_{\mathrm{SEC}} \times \log _{2}(M) \times n_{c}}{C_{\mathrm{S}} \times n_{a}} .
$$

The average system throughput $\bar{\eta}_{\mathrm{SYS}}=E\left[\eta_{\mathrm{SYS}}\right]$ is obtained by averaging over many MUs, or equivalently by using $\overline{\mathrm{P}}_{e}$ instead of $\mathrm{P}_{e}$ in (3.24). The cumulative distribution function (CDF) of the system throughput is given by $\operatorname{Pr}\left(\eta_{\mathrm{SYS}} \leq \eta\right)$ which is the probability that the system throughput takes on a value less than or equal to $\eta$. Finally the system outage probability, $\mathrm{P}_{\mathrm{OUT}}$, is evaluated from the CDF according to

$$
\mathrm{P}_{\mathrm{OUT}}=\operatorname{Pr}\left(\eta_{\mathrm{SYS}} \leq \eta_{\mathrm{OUT}}\right)
$$

where $\eta_{\text {OUT }}$ is the outage throughput which is an arbitrarily chosen value for $\eta_{\text {SYS }}$ below which the system is considered in outage.

\subsection{Simulation Algorithm and Parameters}

Computer simulations were used to evaluate the $\overline{\mathrm{PER}}_{i \mathrm{RSs}}^{(1)}$ and $\overline{\mathrm{PER}}_{i \mathrm{RSs}}^{(2)}$ terms, which are necessary to calculate $\bar{\eta}_{\mathrm{SYS}}$, the throughput CDF, and the outage probability. In this section first the environment parameters and assumptions used in the simulations are presented, followed by an outline of the algorithm used to run the simulations. 


\subsubsection{Environment Parameters and Assumptions}

Below is a list of parameters used in the simulation algorithms. These parameters were inspired by, but not limited to, suggested parameters of the WINNER [26] project for urban macro cellular applications outlined in the public documents [27] and $[28]$.

- Downlink scenario

- Pathloss propagation exponent: $\rho=3.5$

- Log normal shadowing with a standard deviation of $\sigma_{S}=8 \mathrm{~dB}$.

- Frequency selective fading given by the SUI-5 channel model as illustrated in Section 2.6.

- Slow fading, due to the envisioned high data rate coverage. Fast fading typically only occurs for very low data rate applications [2].

- Doppler effects are ignored, since the effects of the Doppler spread is expected to be negligible for slow fading channels [2].

- Carrier frequency: $f=3.95 \mathrm{GHz}$.

- Close-in reference distance: $d_{0}=1$ meter.

- Mean path-loss model by evaluating (3.1): $\bar{L}_{P}(d)=44.373+35 \log (d)$

- Transmission bandwidth: $W=5 \mathrm{MHz}$.

- System temperature: $T_{K}=300^{\circ}$ Kelvin. 
- Thermal noise power by evaluating (3.6): $N_{T}=-101.8403 \mathrm{dBm}$.

- Mobile unit receiver noise figure: $N_{F}=5 \mathrm{~dB}$.

- Antenna Gains:

- Directional BSS mainlobe antenna gain for three-sectored cells $G_{\mathrm{DA}_{\mathrm{BSS}}}=$ $14 \mathrm{~dB}$.

* Maximum attenuation: $A_{\max }=20 \mathrm{~dB}$

$* 3 \mathrm{~dB}$ beamwidth: $\theta_{3 \mathrm{~dB}}=70^{\circ}$

-- Equivalent omnidirectional BSS antenna gain for single-sectored cells calculated from Section 3.3.2 $G_{\mathrm{OA}_{\mathrm{BSS}}}=11.743 \mathrm{~dB}$.

- Omnidirectional RS antenna gain $G_{\mathrm{OA}_{\mathrm{RS}}}=9 \mathrm{~dB}$.

- Transmit powers:

- BSS transmit power: $P_{t_{\mathrm{BSS}}}=46 \mathrm{dBm}$.

- RS transmit power: $P_{t_{\mathrm{RS}}}=37 \mathrm{dBm}$.

- Number of simulation runs: $1,000,000$ MUs placed randomly in the central BSS.

- Cell radius: $\mathrm{R}=100,200, \cdots, 1000$ meters.

- Number of available relays per cell: $N_{\mathrm{R}}=3,6$.

- Simulation area: first tier of co-channel interfering hexagonal cells, 1-cell and 4-cell clusters as shown in Section 3.4. According to variations of $C_{\mathrm{S}}, N_{\mathrm{SEC}}$, $N_{\mathrm{R}}$, and $N_{\mathrm{CELL}}$, the following layouts can be defined 
Table 3.3: Cellular Layouts.

\begin{tabular}{|c|c|c|c|c|c|}
\hline Cellular layout description & $C_{\mathrm{S}}$ & $N_{\text {CELL }}$ & $N_{\text {SEC }}$ & $N_{\text {BSS }}$ & $N_{\mathrm{R}}$ \\
\hline Single 1-sector cell & - & 1 & 1 & 1 & 3 \\
\hline Single 1-sector cell & - & 1 & 1 & 1 & 6 \\
\hline Single 3-sectored cell & - & 1 & 3 & 3 & 3 \\
\hline Single 3-sectored cell & - & 1 & 3 & 3 & 6 \\
\hline Multiple 1-sector cells & 1 & 7 & 1 & 7 & 3 \\
\hline Multiple 1-sector cells & 1 & 7 & 1 & 7 & 6 \\
\hline Multiple 3-sectored cells & 1 & 7 & 3 & 21 & 3 \\
\hline Multiple 3-sectored cells & 1 & 7 & 3 & 21 & 6 \\
\hline Multiple 1-sector cells & 4 & 7 & 1 & 7 & 3 \\
\hline Multiple 1-sector cells & 4 & 7 & 1 & 7 & 6 \\
\hline Multiple 3-sectored cells & 4 & 7 & 3 & 21 & 3 \\
\hline Multiple 3-sectored cells & 4 & 7 & 3 & 21 & 6 \\
\hline
\end{tabular}


- Further assumptions:

- The BSS-RS link is assumed to be reliable enough so that it can perfectly receive the BSS transmission on the first hop. This assumption is in accordance with the relaying deployment concepts of the WINNER project [29].

- For each run relays are randomly placed in the cell with the same number of relays per sector. So if $N_{\mathrm{R}}=6$ and $N_{\mathrm{SEC}}=3$ there will be two randomly placed relays in each BSS. The random placement of relays in the BSS ensure that the results are not dependent upon a specific location rather they are averaged across all possible locations.

\subsubsection{Simulation Algorithm}

Below is an outline of the simulation algorithm used to evaluate the $\overline{\mathrm{PER}}_{i \mathrm{RSs}}^{(1)}$ and $\overline{\mathrm{PER}}_{i \mathrm{RSs}}^{(2)}$ terms according to the previously mentioned parameters and assumptions. For each simulation run:

1. Place a MU randomly in the central BSS (or equivalently central cell for $N_{\mathrm{SEC}}$ $=1)$

2. Calculate the $\mathrm{MU}$ received power, $P_{r}$, from all BSSs using the link budget formula in (3.4).

3. Place $N_{\mathrm{R}} / N_{\mathrm{SEC}} \mathrm{RSs}$ in the central BSS and choose the RS with strongest signal power to the MU to be the serving RS in the second-hop.

4. Place a single RS randomly located in all other interfering BSSs.

5. Calculate the MU received power from all RSs. 
6. Choose $i$ distinct RSs randomly from all interfering RSs to form the set of $i$ interfering RSs for this run.

7. In the first-hop The desired signal power, $P_{S}$, is equivalent to the received power, $P_{r}$, from the central BSS, while on the second-hop $P_{S}$ is equivalent to $P_{r}$ from the chosen RS in the central BSS.

8. Generate the list of interferers (refer to Tables 3.1, and 3.2) according to the value of $i$, first or second-hop transmission, and whether ORCs or nORCs is used.

9. Sort interferers descendingly according to $P_{r}$ then determine $P_{P}$, and $P_{O}$ for the different schemes (w/o-IC, w-IC, PIC1, PIC3).

10. Calculate $\gamma$ and $\Delta$ for the different schemes as discussed in Section (3.5.3).

11. For each scheme use the $(\gamma, \Delta)$ pair to fetch the PER for that MU from the appropriate the link-level table.

After evaluating the $\overline{\mathrm{PER}}_{i \mathrm{RSs}}^{(1)}$ and $\overline{\mathrm{PER}}_{i \mathrm{RSs}}^{(2)}$ terms, they are used in the calculation of the average probability of packet error, $\overline{\mathrm{P}}_{e}$, which is then used to calculate the average system throughput $\left(\bar{\eta}_{\mathrm{SYS}}\right)$.

To evaluate the system throughput CDF, which is also used to determine the system outage probability, the $\overline{\mathrm{PER}}_{i \mathrm{RSs}}^{(1)}$ terms were used to evaluate the average probability of packet error on the first-hop, $\overline{\mathrm{P}}_{e, 1}$, which is equivalent to the probability of relaying. According to this probability of relaying, the number of interfering RSs, if any, were determined for each MU. For example, on the second-hop a uniformly distributed random variable, $\chi \sim \mathrm{U}(0,1)$, is used for each BSS other than the central 
BSS. If $\chi \leq \overline{\mathrm{P}}_{e, 1}$ then this BSS will have an interfering RS. After deciding upon the total number of interfering RSs for this specific MU, the instantaneous system throughput can be calculated which is used in the calculation of the CDF and the probability of outage. 


\section{Chapter 4}

\section{Simulation Results}

This chapter presents the performance evaluation results by means of computer simulation for the investigated cellular layouts with and without using the IC. First, the results for single-hop networks are shown in Section 4.1. Then the results for two-hop networks are discussed in Section 4.2. Single and multiple-cell scenarios are discussed for both kinds of networks.

The single-cell scenario corresponds to a situation where the number of subscribers in a certain geographic area is limited, like a small town or village hence limiting the need for reusing frequencies by installing more cell sites. The multiplecells scenario on the other hand is more likely to be found in cities and metropolitan areas where more cell sites are used to accommodate the increase in the number of subscribers through reusing frequencies.

In this chapter the performance of each setup is investigated in a number of ways. First we look at the average system throughput $\left(\bar{\eta}_{\mathrm{SYS}}\right)$ for different cell sizes. Also each setup is evaluated in terms of the system outage probability $\left(\mathrm{P}_{\mathrm{OUT}}\right)$, where the outage throughput, $\eta_{\mathrm{OUT}}$, is chosen to be 0.12 bpchu. This value was chosen arbitrarily due to availability of the results at this threshold for all schemes. Finally for the single-hop section we also showed the throughput CDF at a cell size $(R)$ of 500 meters. 


\subsection{Single Hop}

In this section we discuss the results for single-hop networks. The objective of this section is to show the different benefits that the IC can provide in such networks. We Use the single BSS per cell without interference cancellation, $N_{\mathrm{SEC}}=1(\mathrm{w} / \mathrm{o}-\mathrm{IC})$, as a reference scheme. This reference scheme is compared to $N_{\mathrm{SEC}}=3$ (w/o-IC) and (w-IC) to investigate the effect of sectoring, using three co-channel BSSs per cell with directional antennas, on the system performance without and with the IC respectively.

For the multiple-cell scenario, in addition to looking at the effect of sectoring, we also look at the effectiveness of using the IC to combat CCI from other cells. This is done by first comparing the reference scheme, $N_{\mathrm{SEC}}=1$ (w/o-IC), in both single and multiple cell scenarios to show the effect of the added CCI from adjacent cells, then comparing that to $N_{\mathrm{SEC}}=1$ (w-IC) in the multiple-cell scenario to show the role of the IC in mitigating the CCI effect. The performance parameters used in this section are the average system throughput in bpchu for different cell sizes (different values of $R$ ), the CDF of the throughput at $R=500$ meters, and the system outage probability for different cell sizes evaluated for $\eta_{\mathrm{OUT}}=0.12 \mathrm{bpchu}$.

\subsubsection{Single-Cell Scenario}

The average system throughput, as a function of the cell radius, is shown in Figure 4.1. For the unsectorized case, $N_{\mathrm{SEC}}=1,(\mathrm{w}-\mathrm{IC})$ and $(\mathrm{w} / \mathrm{o}-\mathrm{IC})$ are identical since there is no interference to cancel. However, for $N_{\mathrm{SEC}}=3$ and (w/o-IC), a lot more CCI is introduced into the system resulting in a 50\% drop in average system throughput for the (w/o-IC) system. Although sectoring nominally increases the throughput 
because three users can be served simultaneously, because the same channel is used in all sectors the co-channel interference is very severe, which may lead to a drop in the actual performance. If the IC is used, however, the CCI can be reduced very effectively, leading to an average system throughput that is at least double that of the unsectorized case.

The CDF of the system throughput is shown in Figure 4.2. Without the use of the IC, it can be seen that for more than $50 \%$ of the time the system throughput in the sectorized case $\left(N_{\mathrm{SEC}}=3\right)$ is less than half the system nominal throughput of the unsectorized case (which is $1 \mathrm{bpchu}$ ). On the othr hand, by employing the IC, the sectorized case is able to deliver throughput greater than the unsectorized nominal throughput about $95 \%$ of the time, and double the nominal throughput $85 \%$ of the time.

The impact of sectoring on the outage probability is evident in Figure 4.3. Without the IC, the outage probability increases significantly in the sectorized case. For example, at $R=500$ meters the outage probability is $40 \%$, compared to $1.4 \%$ for the unsectorized case. However, by using the IC, the impact of sectorizing on the outage probability is very minor, increasing only to $1.8 \%$ at $R=500$ meters. 


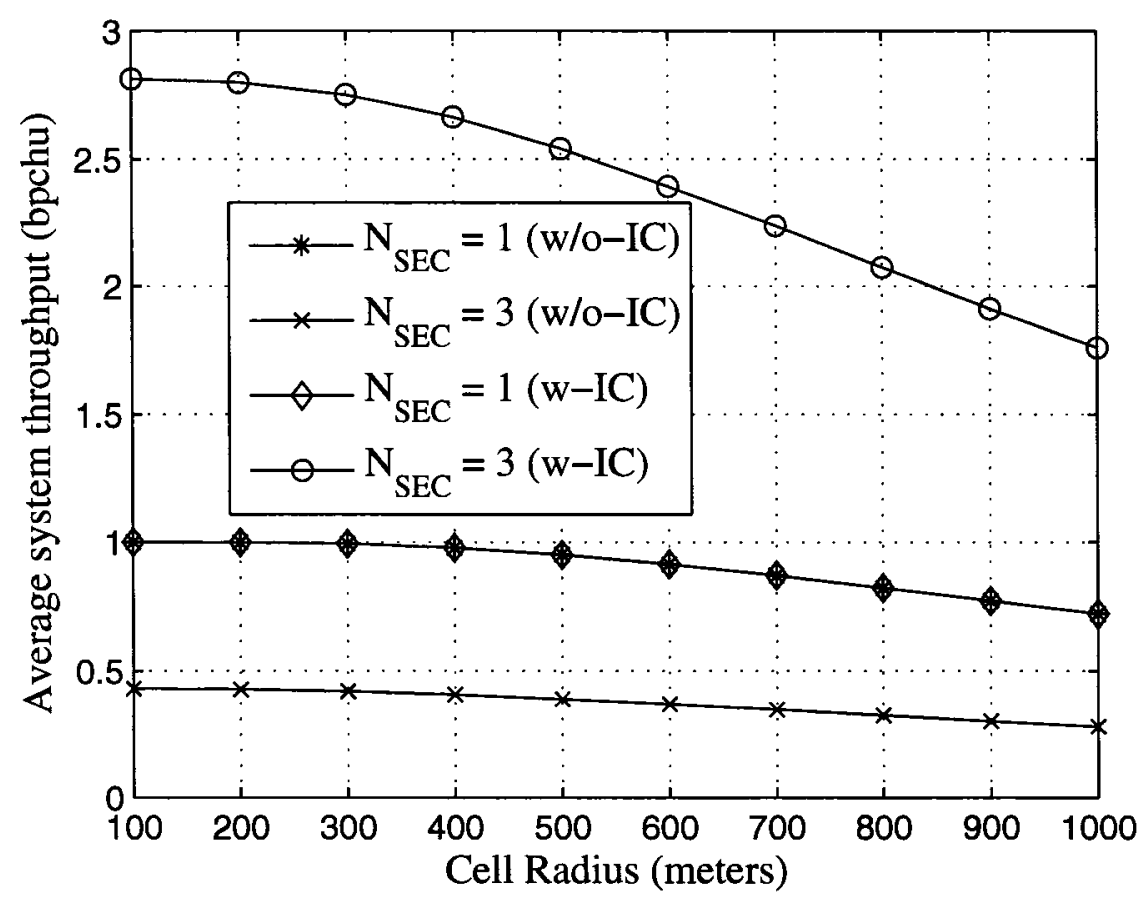

Figure 4.1: The average system throughput in (bpchu) for single-hop networks and $N_{\mathrm{CELL}}=1$.

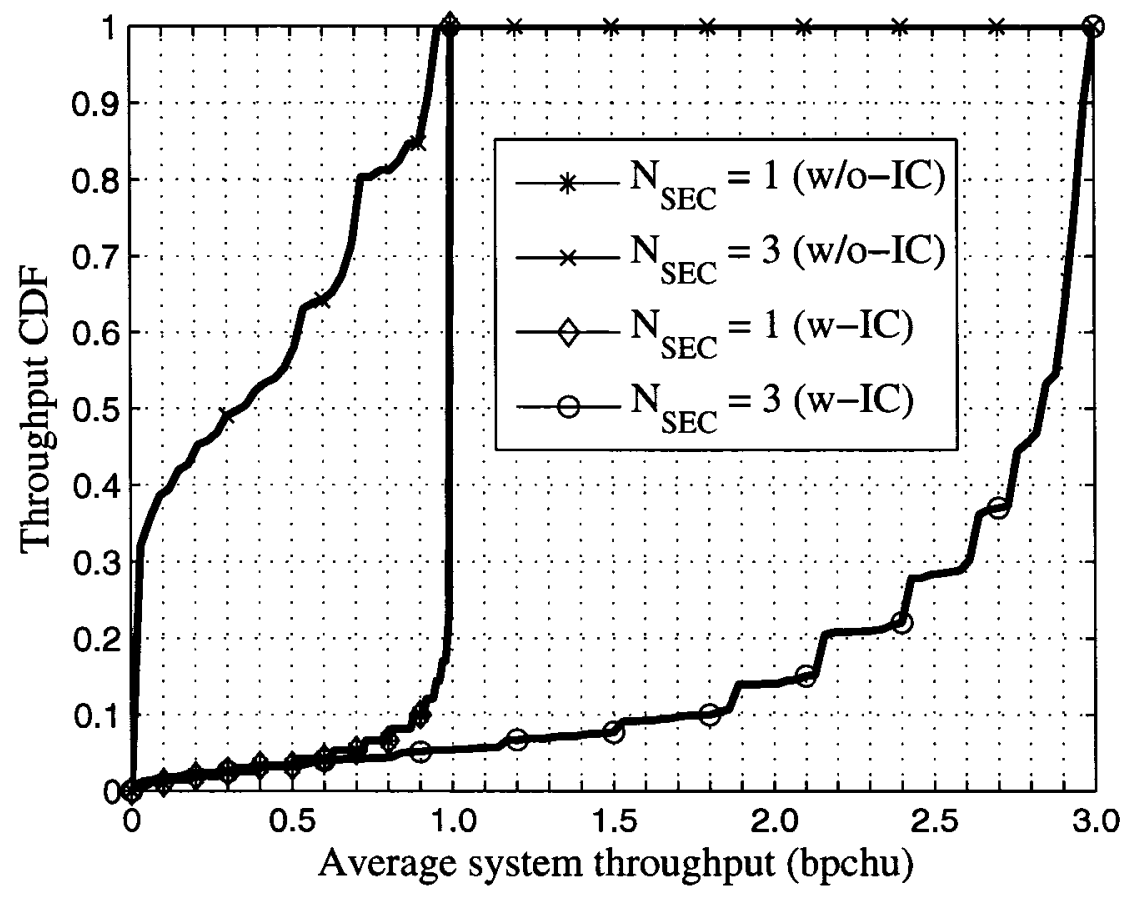

Figure 4.2: The throughput CDF at $R=500$ meters for single-hop networks with $N_{\mathrm{CELL}}=1$. 


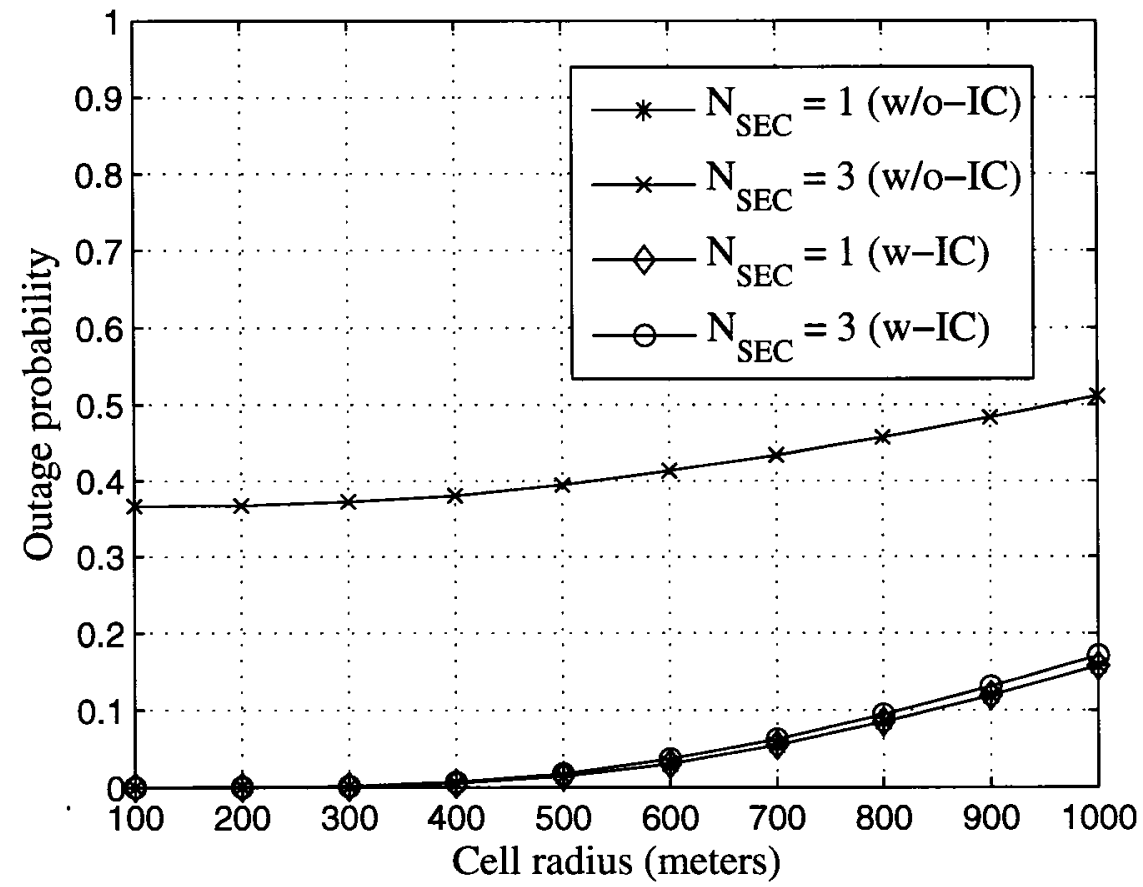

Figure 4.3: The outage probability for single-hop networks with $N_{\mathrm{CELL}}=1$. 


\subsubsection{Multiple-Cell Scenario}

For the multiple-cell scenario, we start by looking at the effect of CCI from adjacent cells on the reference scheme, by comparing $N_{\mathrm{SEC}}=1(\mathrm{w} / \mathrm{o}-\mathrm{IC})$ for both single and multiple-cell scenarios. For $C_{\mathrm{S}}=1$, if we compare Figures 4.1 and 4.3 for single-cell to Figures 4.4 and 4.6 for multiple-cell, we can see that due to the CCI from surrounding cells the reference scheme experiences $85 \%$ to $89 \%$ reduction in average system throughput together with a significant increase in outage probability. With the IC, although the perormance is still inferior to that of the single-cell scenario, there is close to four fold increase in average system throughput and $50 \%$ reduction in outage probability compared to $N_{\mathrm{SEC}}=1$ (w/o-IC) in the multiple-cell scenario indicating the effectiveness of the IC in mitigating the CCI effects.

Now we focus on the results of the multiple-cell scenario, shown in Figures 4.4 to 4.6 for $C_{\mathrm{S}}=1$ and Figures 4.7 to 4.9 for $C_{\mathrm{S}}=4$. Without the IC, sectoring results in less average system throughput coupled with higher outage probability for both cluster sizes. For example, for $C_{\mathrm{S}}=1$, the average throughput drops from 0.11 bpchu for $N_{\mathrm{SEC}}=1$ to 0.09 for $N_{\mathrm{SEC}}=3$ while the outage probability is increased from $82 \%$ to $84 \%$. Using the IC provides close to eight fold icrease in average system throughput compared to $N_{\mathrm{SEC}}=1$ (w/o-IC) bringing the throughput up to 0.86 bpchu, while reducing the outage probability by over $40 \%$. Also, if we compare $N_{\mathrm{SEC}}=1(\mathrm{w}-\mathrm{IC})$ and $N_{\mathrm{SEC}}=3(\mathrm{w}-\mathrm{IC})$, we see that sectoring results in double the average system throughput while the outage probability is only increased by $6.67 \%$. Finally we observe that with the IC, clustering reduces the outage probability more effciently. For example, comparing $N_{\mathrm{SEC}}=1$ with and without IC in Figures 4.6 and 4.9 for $C_{\mathrm{S}}=1$ and 4 respectively, we see that without IC clustering reduces the outage probability by $18 \%$ only, while with the IC the outage probability is reduced 
by around $50 \%$. 


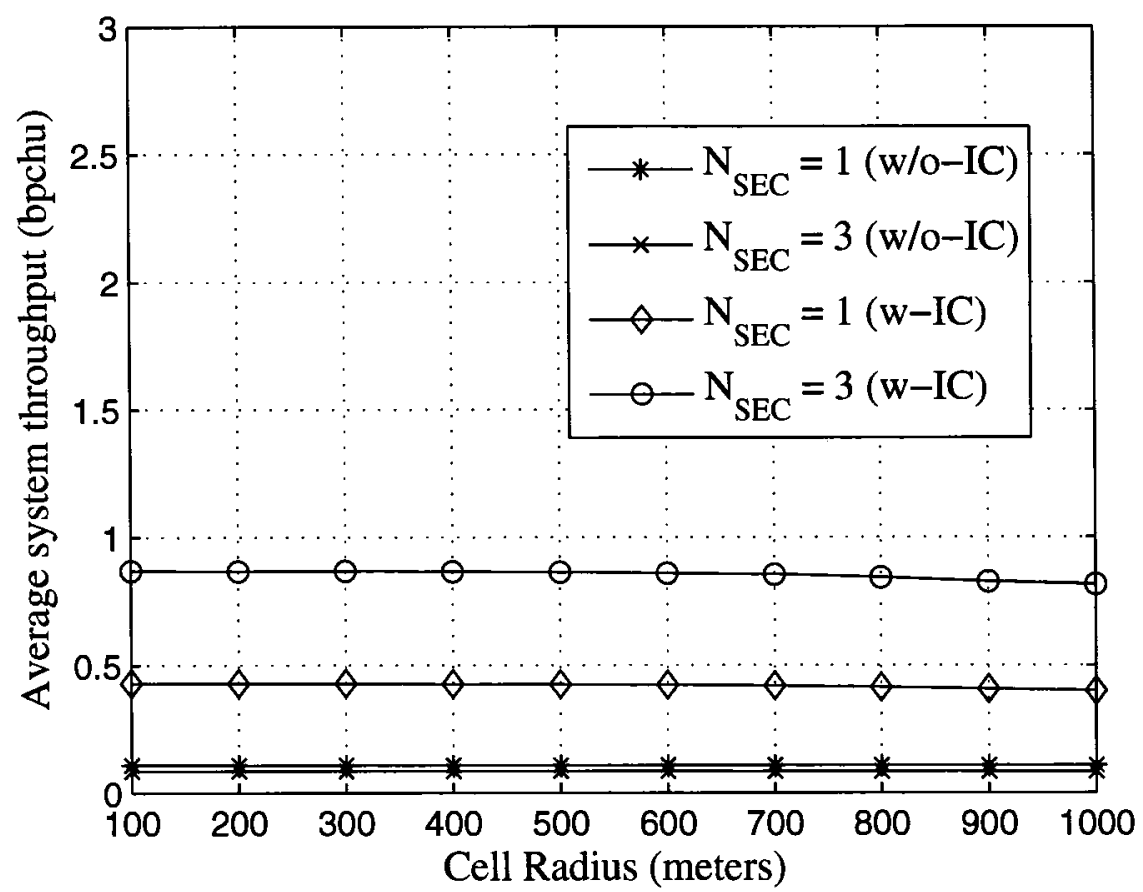

Figure 4.4: The average system throughput in (bpchu) for single-hop, $N_{\mathrm{CELL}}=7$, and $C_{\mathrm{S}}=1$ networks.

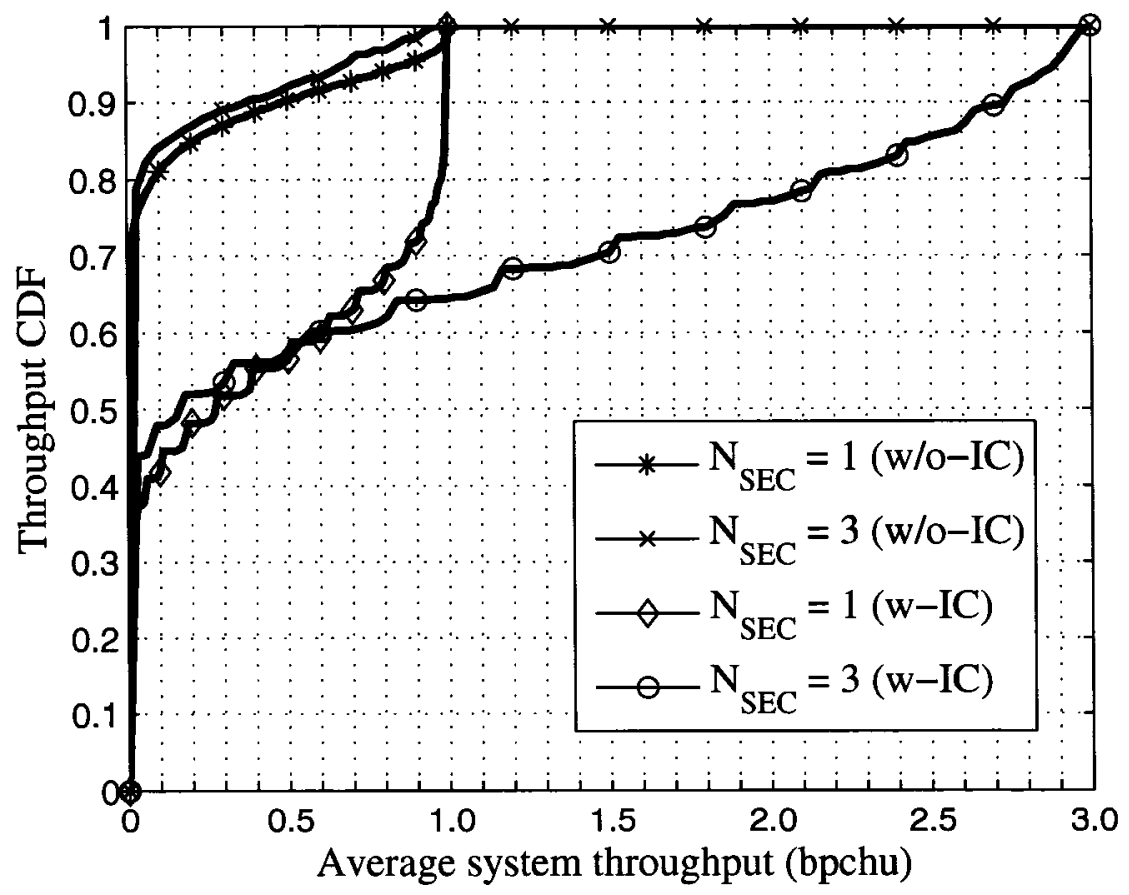

Figure 4.5: The throughput CDF at $R=500$ meters for single-hop, $N_{\mathrm{CELL}}=7$, and $C_{\mathrm{S}}=1$ networks. 


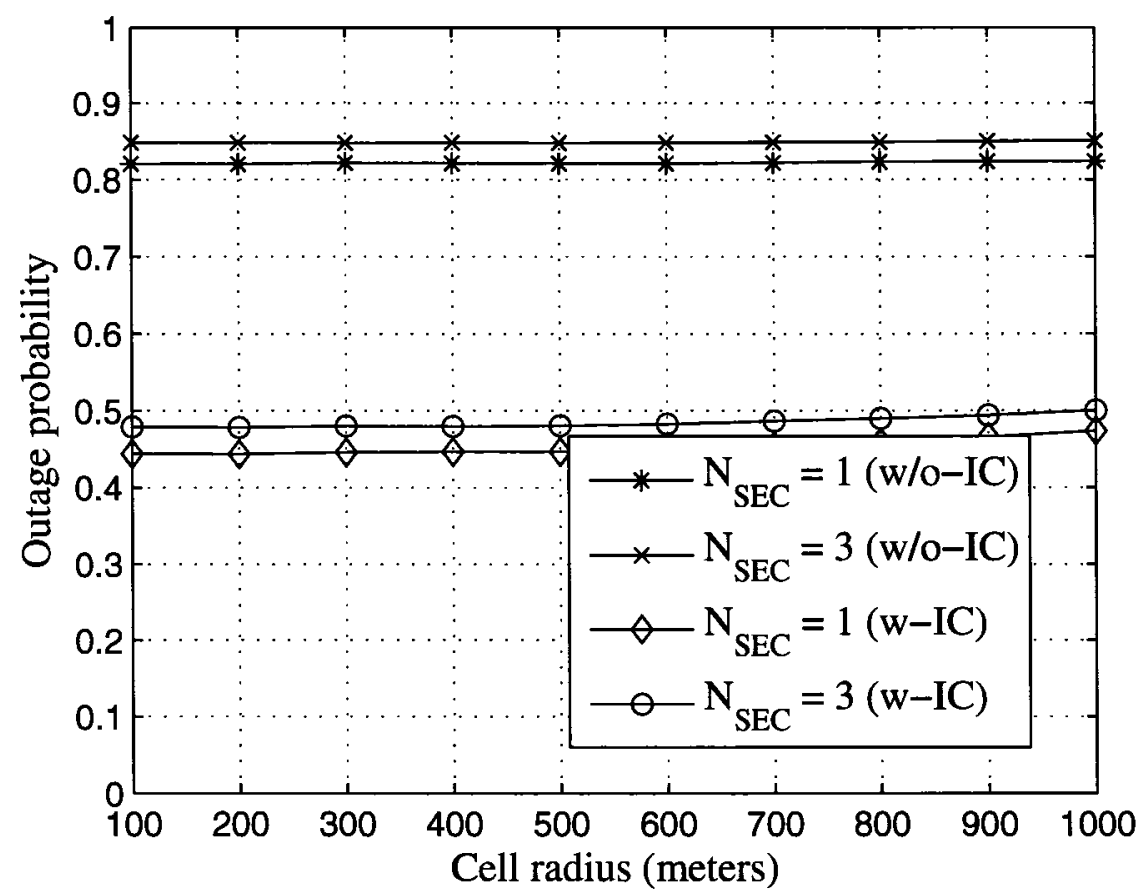

Figure 4.6: The outage probability for single-hop, $N_{\mathrm{CELL}}=7$, and $C_{\mathrm{S}}=1$ networks.

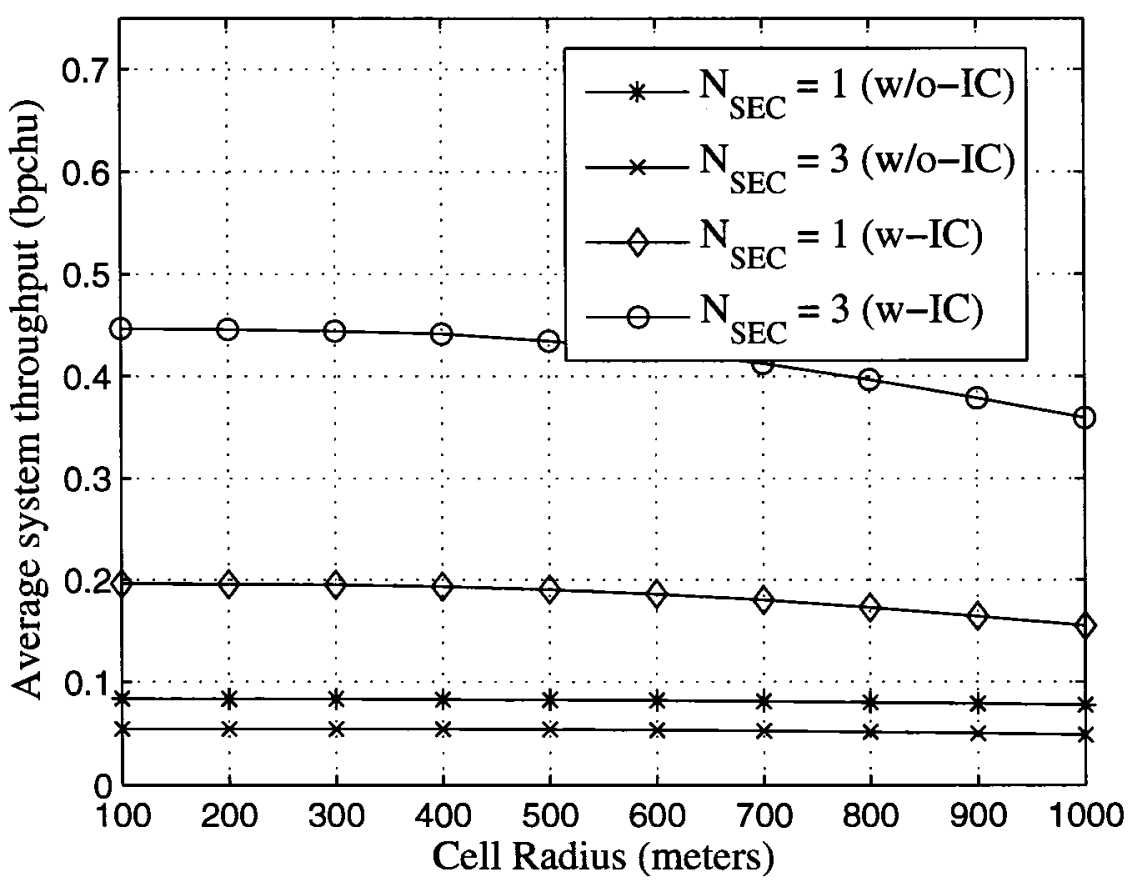

Figure 4.7: The average system throughput in (bpchu) for single-hop, $N_{\text {CELL }}=7$, and $C_{\mathrm{S}}=4$ networks. 


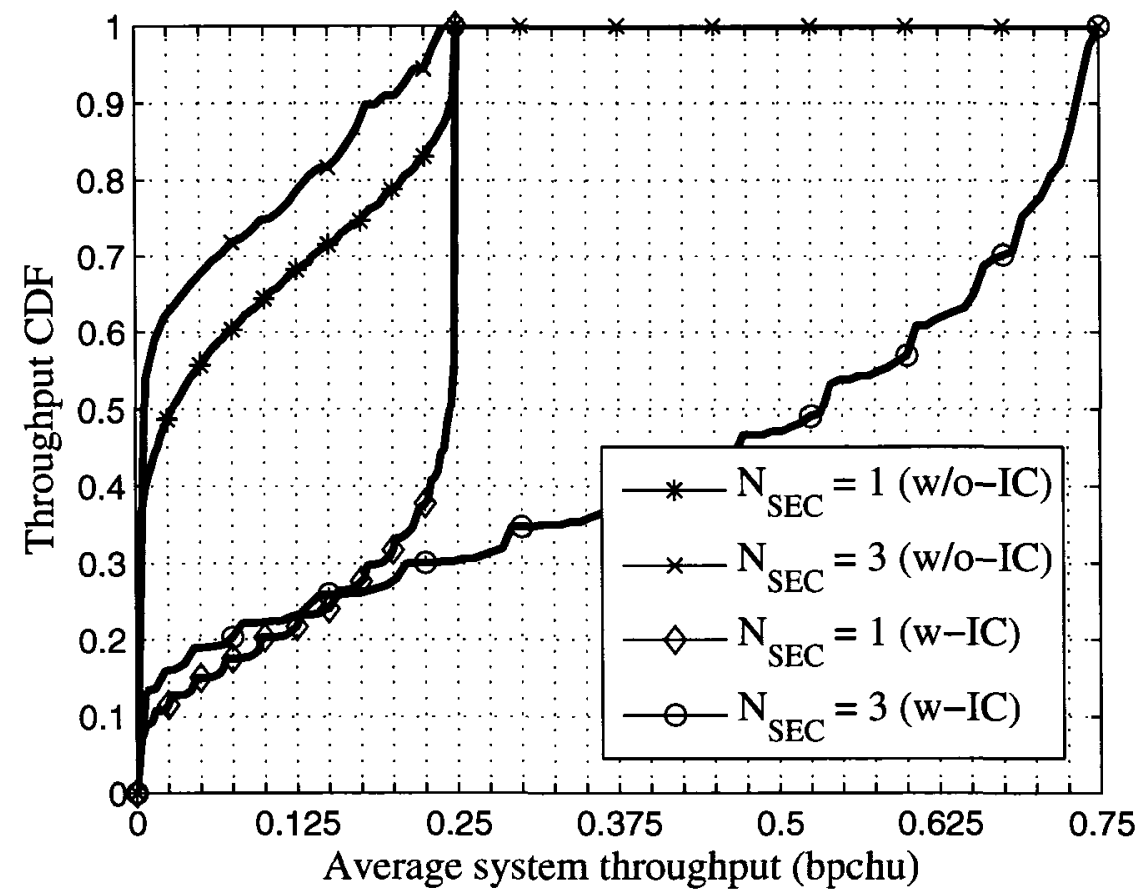

Figure 4.8: The throughput CDF at $R=500$ meters for single-hop, $N_{\mathrm{CELL}}=7$, and $C_{\mathrm{S}}=4$ networks.

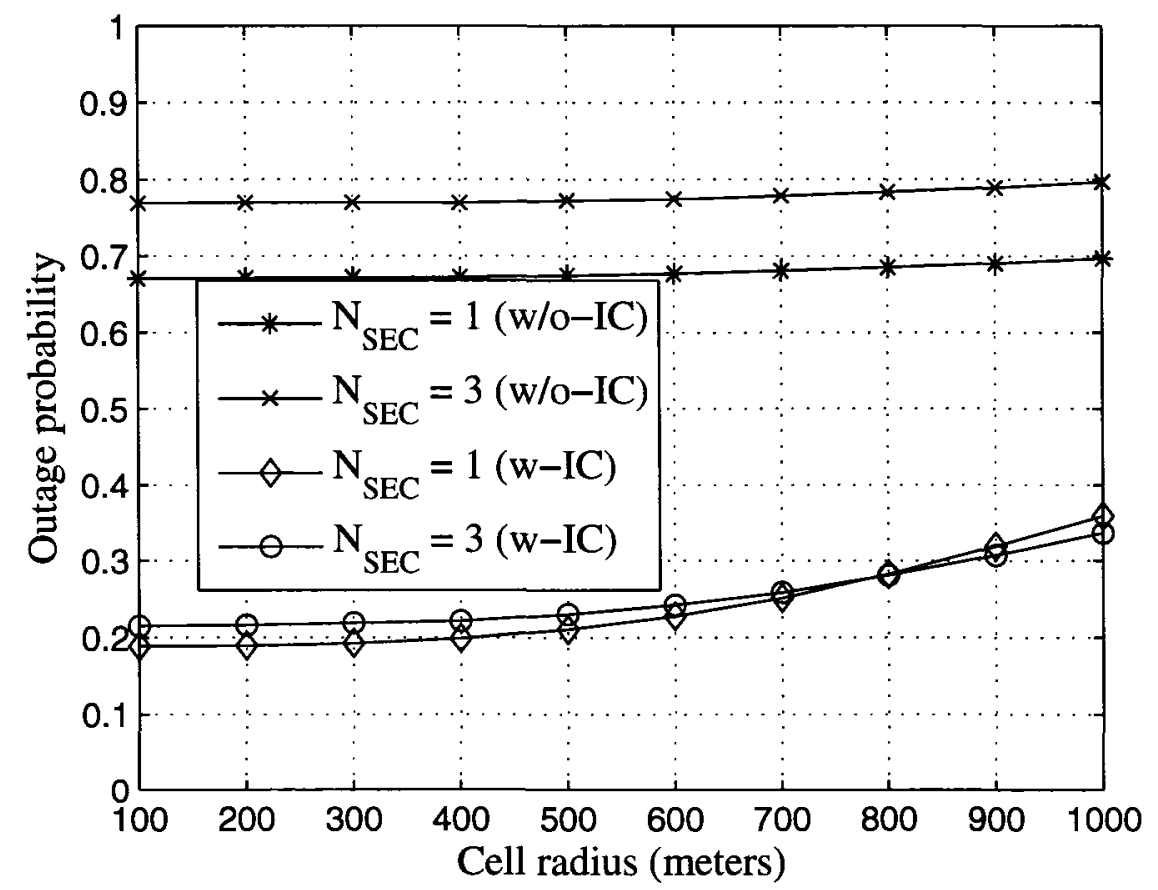

Figure 4.9: The outage probability for single-hop, $N_{\mathrm{CELL}}=7$, and $C_{\mathrm{S}}=4$ networks. 
Therefore, the benefits of the IC for single-hop networks can be summarized in the following.

- Without using the IC, sectoring leads to a degradation in both average system throughput as well as the system outage probability compared to the single BSS per cell without interference cancellation reference scheme. However, with the IC, sectoring achieves a significant increase in average system throughput, in orders of magnitude, for both single and multiple-cell scenarios. This is achieved without sacrificing the system outage probability for the single-cell scenario and with a lower outage probability than the reference scheme for the multiple-cell scenario.

- For the multiple-cell scenario and using a single BSS per cell, the IC is very effective in eliminating CCI from adjacent cells and hence providing a significant average throughput increase as well as a significant reduction in outage probability.

- With the IC, the outage probability reduction achieved by clustering, using $C_{\mathrm{S}}=4$ instead of 1 , is more than the reduction achieved without the IC. 


\subsection{Two-Hop Relay Networks}

In this section we discuss the results for two-hop relay networks. Relaying using ORCs is discussed in Section 4.2.1 while relaying using nORCs is discussed in Section 4.2.2. Finally, in Section 4.2 .3 we look at the effect of the IC on the benefits of relaying by comparing the single-hop (w-IC) case to the relaying schemes with the IC as well. In this section we limit or discussion to the average system throughput and the outage probability.

\subsubsection{Multihop Using Orthogonal Relaying Channels (ORCs)}

When relaying is performed using ORCs the system nominal throughput is reduced by $50 \%$ due to the use of an extra channel. Therefore, regardless of the improvement that relaying can provide in terms of the probability of outage, the probability of error has to be signficantly reduced to compensate for this reduction in the system nominal throughput so that relaying on ORCs can provide throughput enhancement as well. This significant probability of error reduction does not seem feasible without proper interference suppression. Therefore, looking at the performance curves presented in this section we observe the following for ORC relay networks

- Except for single-sector single-cell scenario, relaying without the IC was able to improve both the system average throughput as well as the system outage probability compared to single-hop (w/o-IC). However, these improvements are very modest.

- With the IC, relaying benefits are significantly increased in terms of both the average system throughput and the outage probability. 
- Better results are achieved when more relays are available per cell, i.e. when $N_{\mathrm{R}}$ increases.

- Without the IC, sectoring with relaying provides very poor if any throughput enhancement that does not justify the resulting increase in the probability of outage compared to single-hop (w/o-IC). However, when the IC is used sectoring with relaying provides significant throughput and outage improvement compared to single-hop (w/o-IC) as well.

- In cases where the outage probability is considered to be very high, even with the significant improvement due to using the IC compared to single-hop (w/oIC), reasonably low outage probabilities are expected if the MU has two receive antennas. This speculation is based upon the fact that the IC performance is very close to the perfect interference cancellation of the major interferer (PIC1), which indicates that with two receive antennas the IC performance would also be close to the perfect cancellation of three major interferers (PIC3).

\section{Single-Cell Scenario}

The unsectorized $\left(N_{\mathrm{SEC}}=1\right)$ single-cell case performance results are shown in Figure 4.10. Because there is no CCI, the (w-IC) and (w/o-IC) schemes yield the same performance. We observe the throughput degradation associated with the use of an orthogonal relaying channel, and note that the degradation is relatively less for larger cell sizes. This is because MUs in smaller cells are less likely to require the use of a relayer, but the same price ( $50 \%$ reduction in nominal throughput) is paid to provision the relayers regardless of the cell size. On the other hand, we observe that relaying does reduce the outage probability, particularly in larger cells where we can 
also see an advantage for higher $N_{\mathrm{R}}$ values.

When sectoring is used $\left(N_{\mathrm{SEC}}=3\right), \mathrm{CCI}$ is introduced into the system. If the IC is not used, this leads to a considerable degradation in system performance for the single-hop case, as shown in Figure 4.11. Relaying helps overcome this degradation somewhat, but without the use of the IC, performance, in terms of both throughput and outage probability, remains worse than the unsectorized single-hop case.

By using the IC in conjunction with relaying, however, we see a dramatic improvement. For example $N_{\mathrm{R}}=6$ (w-IC) gives throughput that is roughly 2.5 times greater than $N_{\mathrm{R}}=6$ (w/o-IC), while dropping the outage probability from $26 \%$ to nearly zero for a cell size of 500 meters. The IC is clearly able to reduce the CCI introduced by sectoring, allowing us to enjoy an increase in throughput and a reduction in the outage probability. 


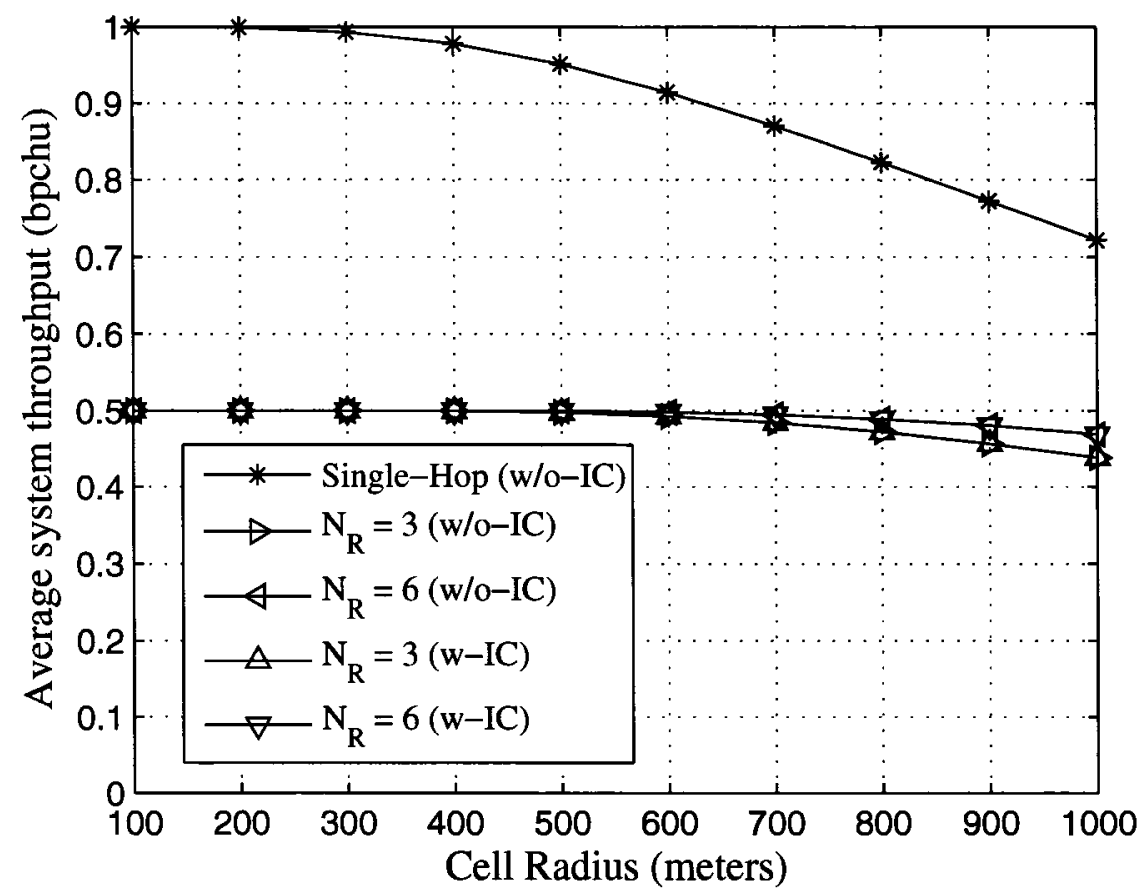

(a) Average system throughput

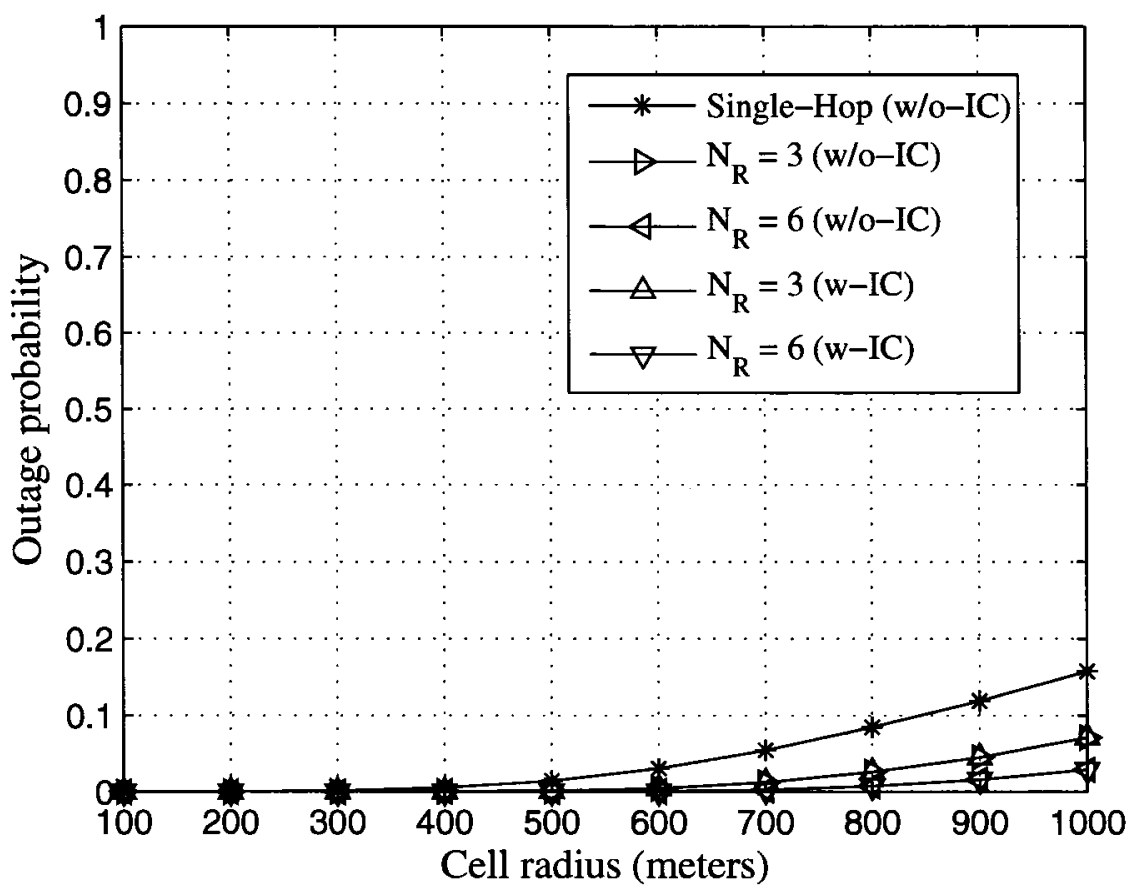

(b) Outage probability

Figure 4.10: System performance for two-hop networks using ORCs, $N_{\text {CELL }}=1$ and $N_{\mathrm{SEC}}=1$. 


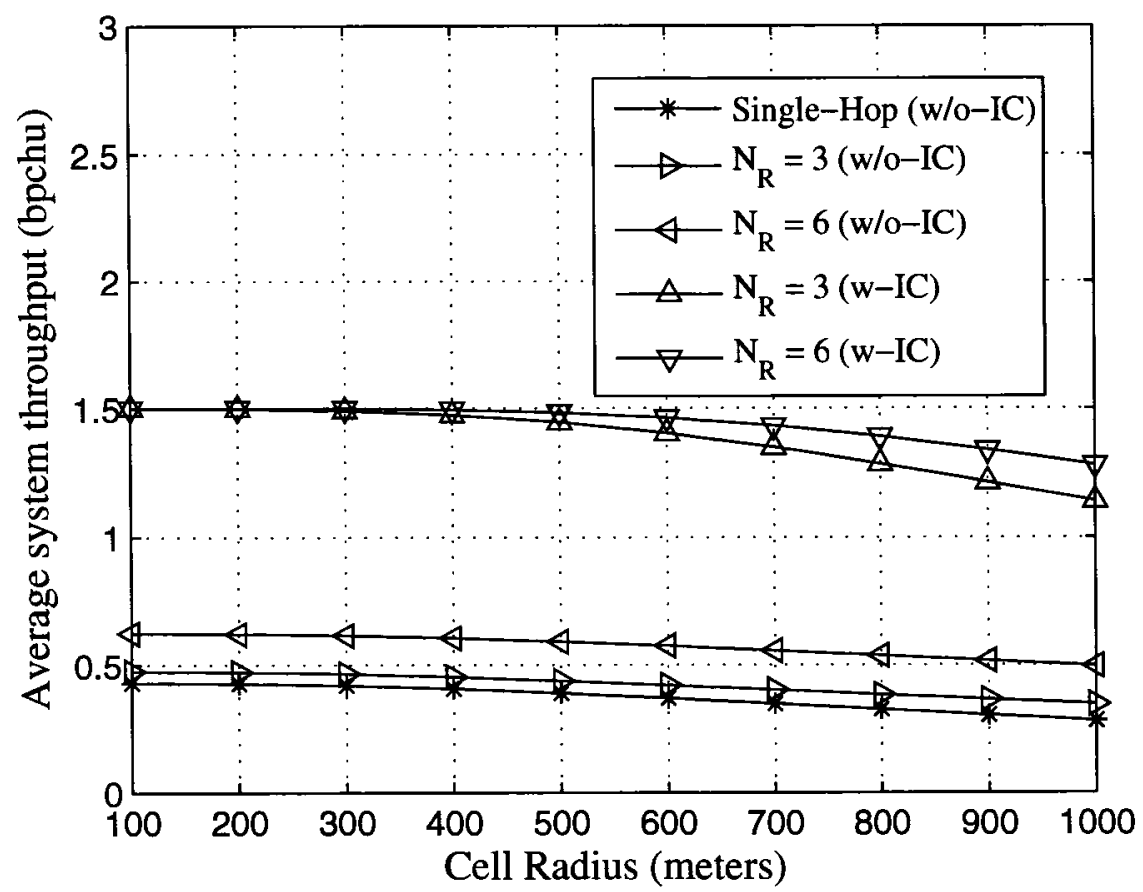

(a) Average system throughput

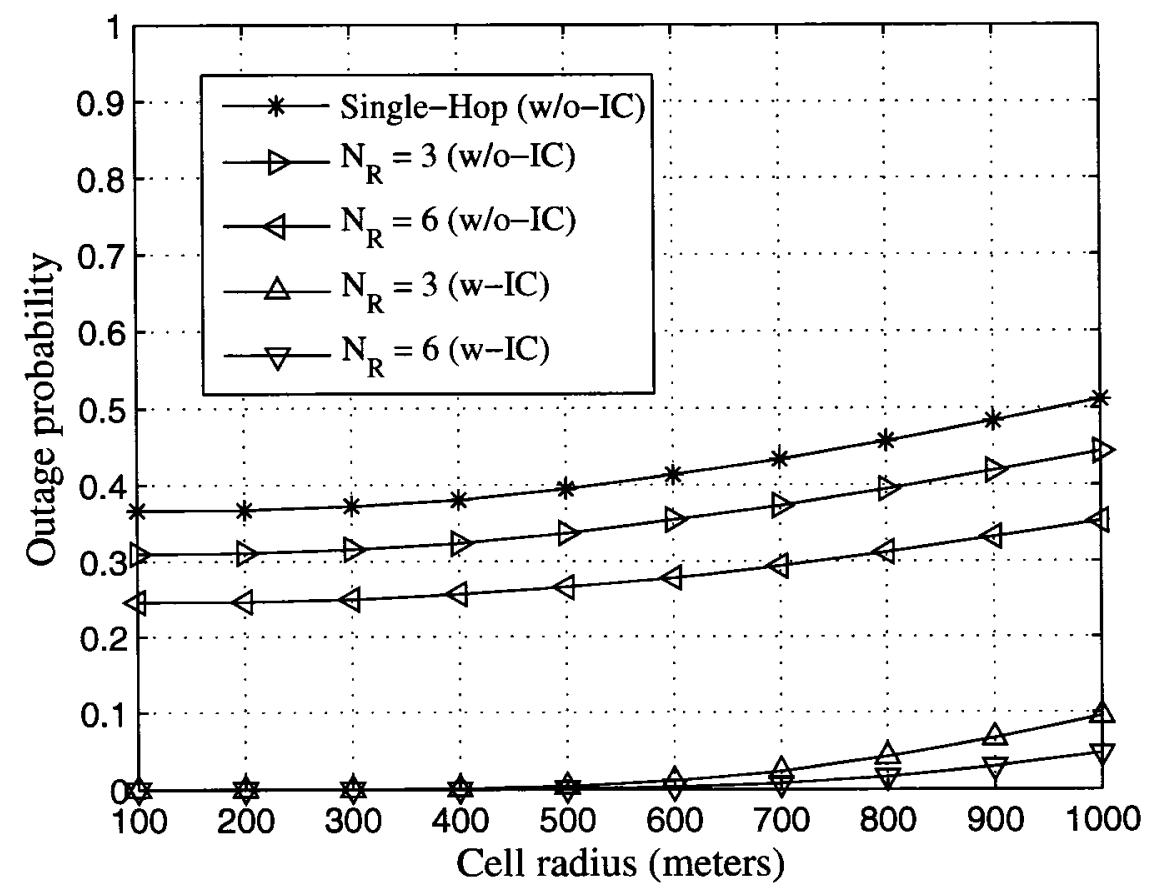

(b) Outage probability

Figure 4.11: System performance for two-hop networks using ORCs, $N_{\text {CELL }}=1$ and $N_{\mathrm{SEC}}=3$. 
The same trends that have been observed for the single-cell scenario are also valid for the multiple-cell scenario. First we can see an advantage for relaying without the IC in both throughput and outage probability, which can be significantly increased if the IC is used. For example, we can see in Figure 4.12, for single-sector cells and a cluster size of one, that $N_{\mathrm{R}}=6$ (w/o-IC) achieves a two fold increase in throughput over single-hop (w/o-IC) which can be increased to a five fold increase if the IC is used, while the outage probability is reduced from $82 \%$ to $47 \%$ for $N_{\mathrm{R}}=6$ (w/o-IC) and to $9 \%$ for $N_{\mathrm{R}}=6$ (w-IC). We also note that better results are achieved for relaying when more relays are available per cell by comparing the curves for $N_{\mathrm{R}}=3$ and $N_{\mathrm{R}}=6$. From this point on, we limit the discussion to the $N_{\mathrm{R}}=6$ case to avoid crowded figures.

The second observation which is also established for the multiple-cell scenario is that sectoring in two-hop networks is not feasible without the IC. For example if we look at Figure 4.13 for cluster size of 4 , there is only a very negligible increase in throughput for relaying (w/o-IC) when sectoring is used $\left(N_{\mathrm{SEC}}=3\right)$, but the outage probability increases from $40 \%$ to $70 \%$. However, with the IC sectoring results in a three fold increase in throughput while outage is raisonably maintained at around $5 \%$. In fact, with sectoring the probability of outage performance of the system is better at higher cell sizes, since for a cluster size of four and $N_{\mathrm{SEC}}=1$, the strength of the interference, and hence the ability of the IC to cancel it, decrease with the cell size increase, while for $N_{\mathrm{SEC}}=3$, interferers from adjacent BSSs in the same cell may still be close and strong enough even with the increase in the cell size. The second observation is also true for cluster size of one, as shown in Figure 4.14, however in that case the throughput gains are accompanied by doubling the outage probability. This 
encouraged us to look at the perfect interference cancellation performance curves (PIC1 and PIC3) shown in Figure 4.15 , for $C_{\mathrm{S}}=1$ and $N_{\mathrm{SEC}}=3$, where we can see that the IC performance is very close to the perfect elemination of the primary interferer (PIC1). This indicates a promising performance close to (PIC3), where the outage probability is reduced from $20 \%$ to $5 \%$ while throughput is increased by $35 \%$, if the MU has two receive antennas, especially when the IC is expected to provide a better interference cancellation with multiple receive antennas as stated by Jie Lin in $[9]$. 


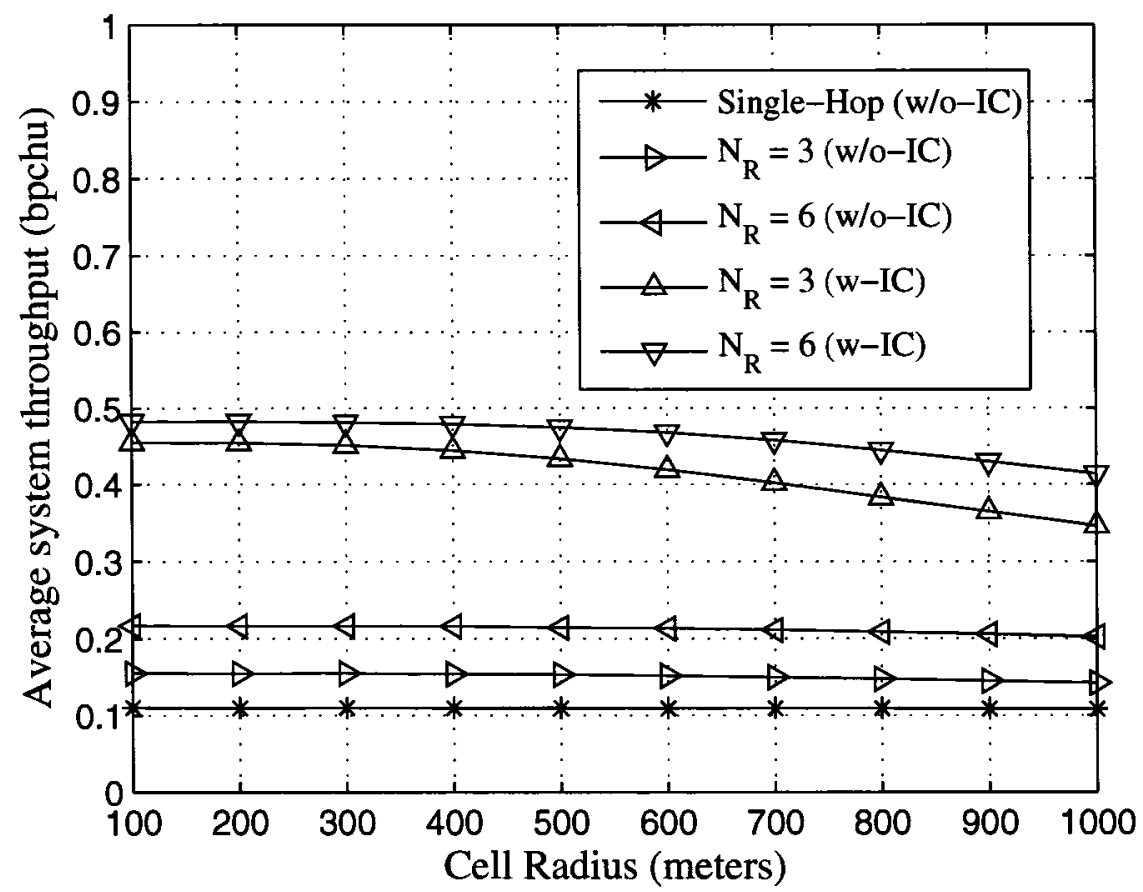

(a) Average system throughput

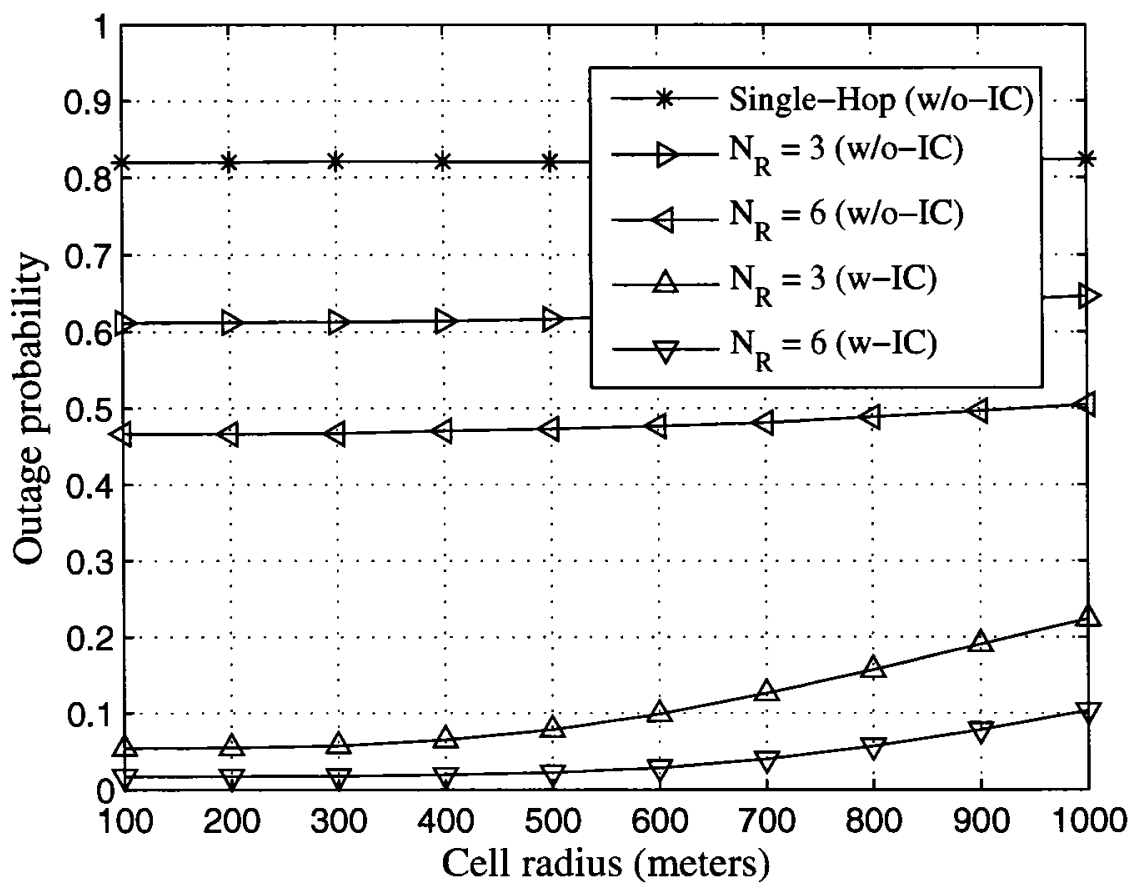

(b) Outage probability

Figure 4.12: System performance for two-hop networks using ORCs, $N_{\text {CELL }}=7$, $N_{\mathrm{SEC}}=1$, and $C_{\mathrm{S}}=1$. 


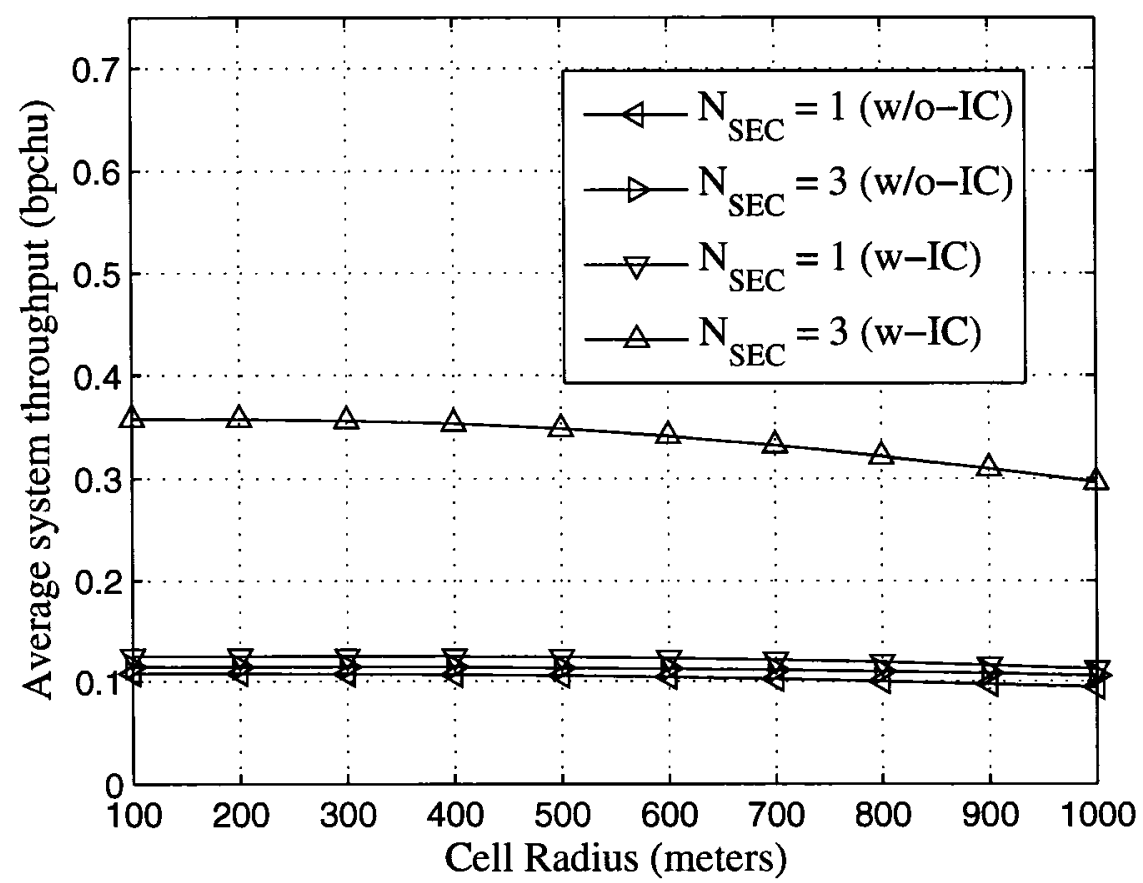

(a) Average system throughput

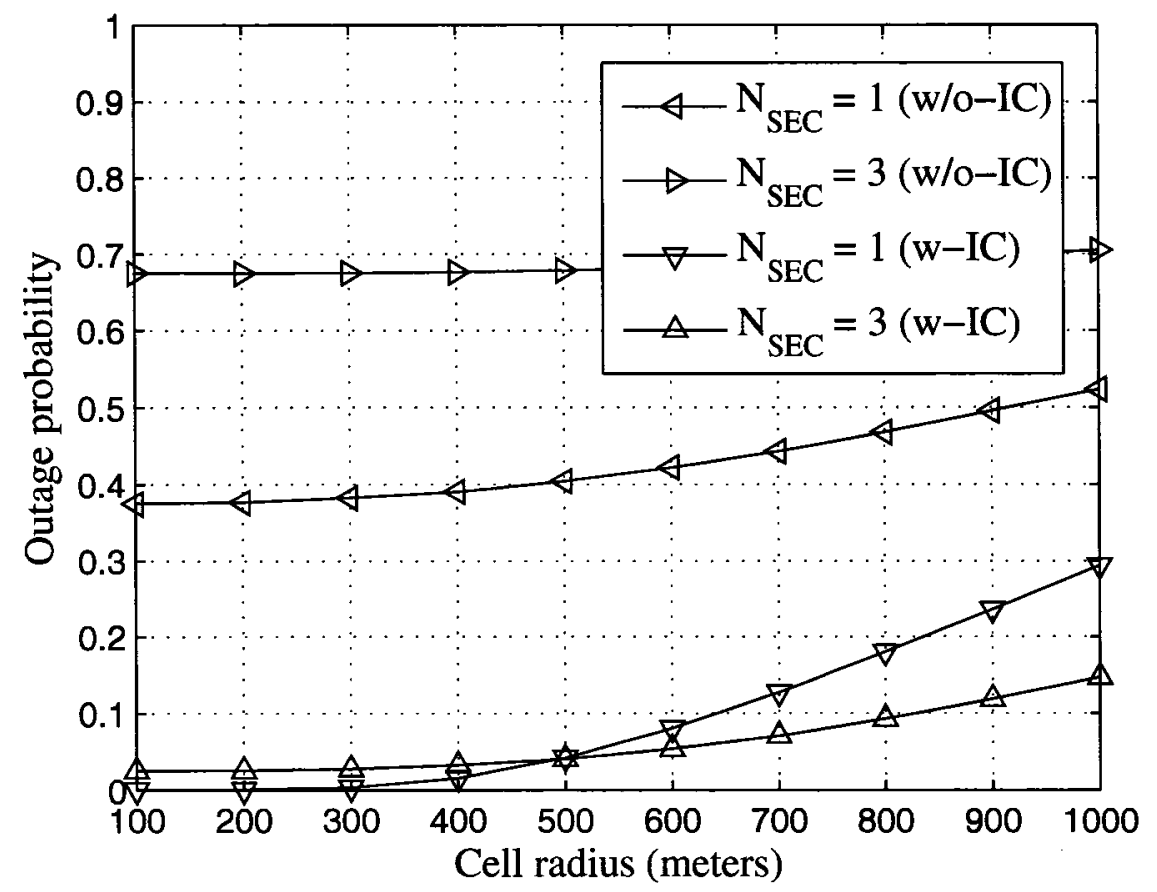

(b) Outage probability

Figure 4.13: System performance for two-hop networks using ORCs, $N_{\mathrm{R}}=6, N_{\mathrm{CELL}}=$ 7 , and $C_{\mathrm{S}}=4$. 


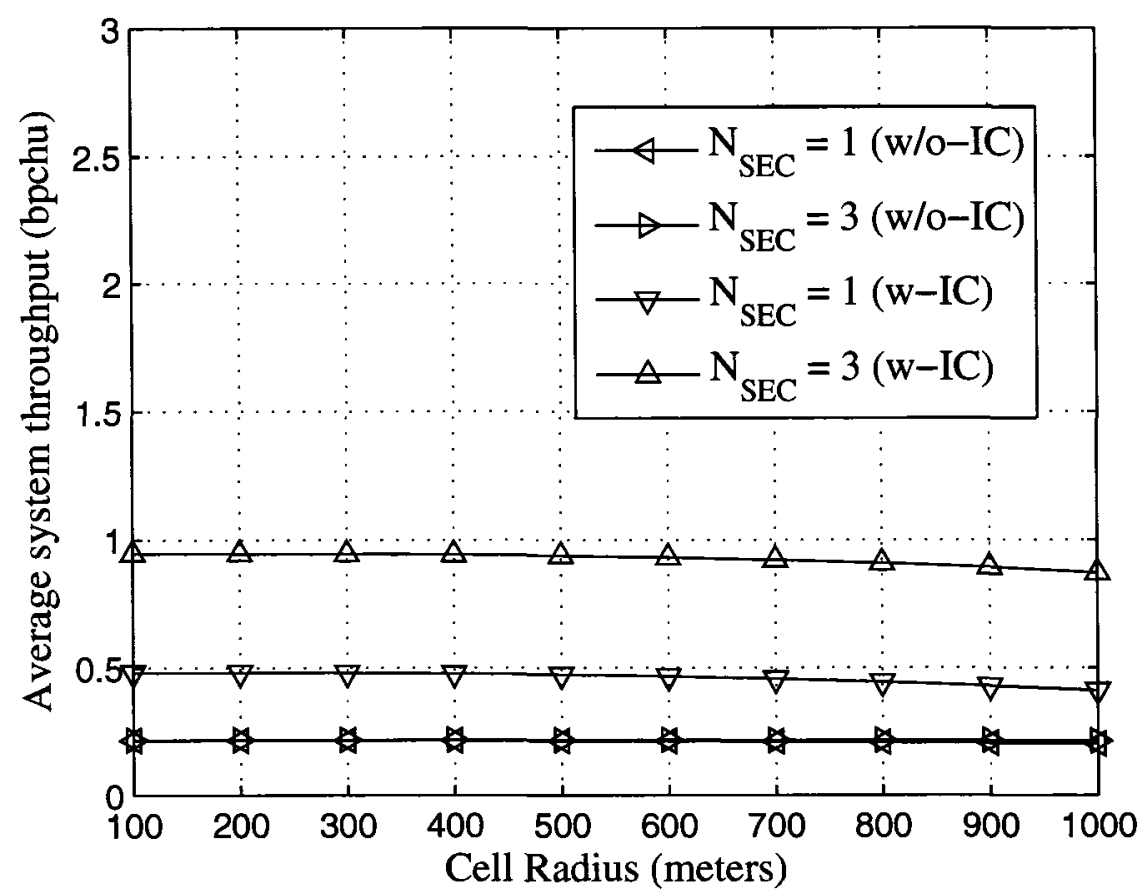

(a) Average system throughput

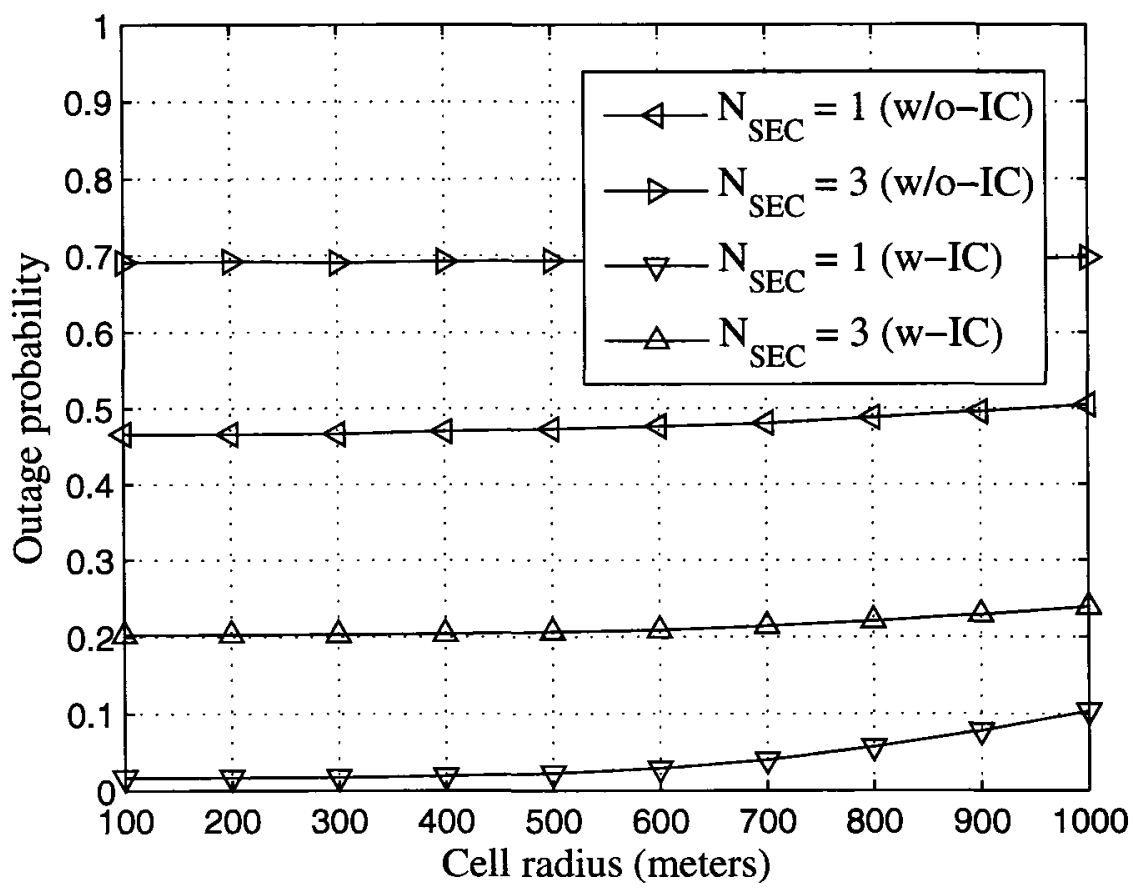

(b) Outage probability

Figure 4.14: System performance for two-hop networks using ORCs, $N_{\mathrm{CELL}}=7$, and $C_{\mathrm{S}}=1$. 


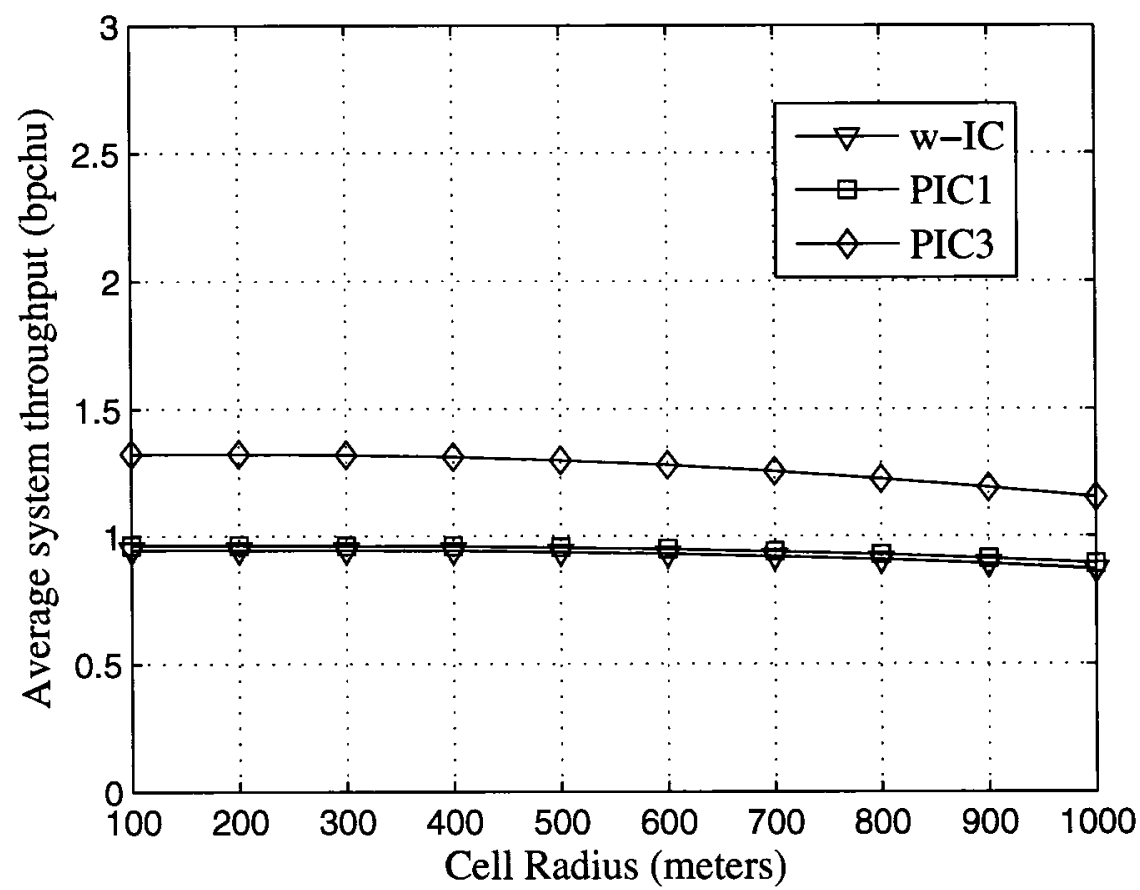

(a) Average system throughput

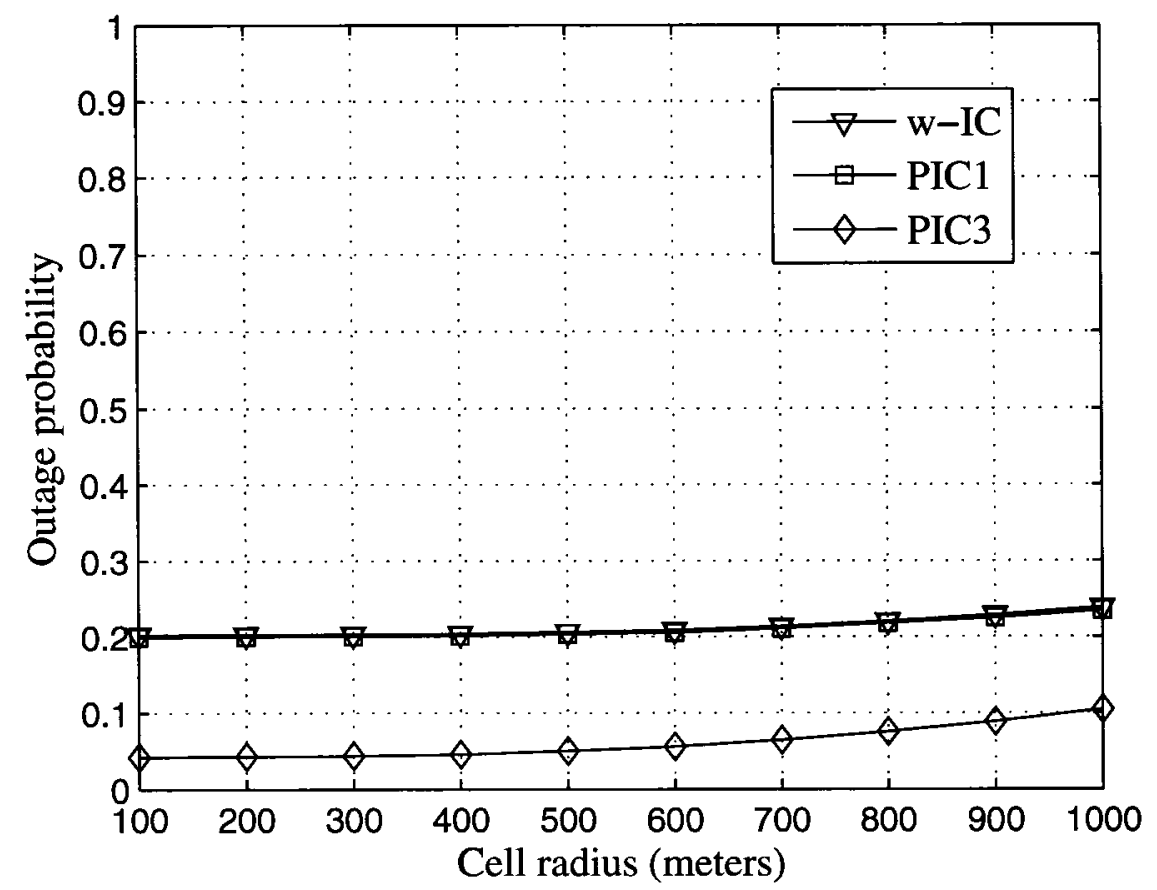

(b) Outage probability

Figure 4.15: System performance with perfect interference cancellation (PIC) for twohop networks using ORCs, $N_{\mathrm{R}}=6, N_{\mathrm{CELL}}=7, N_{\mathrm{SEC}}=3$, and $C_{\mathrm{S}}=1$. 


\subsubsection{Multihop Using non-Orthogonal Relaying Channels (nORCs)}

In the previous section, we have seen how relaying on ORCs can be used to improve the system's outage probability and throughput especially with the use of the IC. However, with ORCs the system suffered from a $50 \%$ reduction in the system nominal throughput, which acted as a ceiling for the system throughput regardless of the reduction in the probability of error. For that reason, we consider relaying using nORCs to remove this initial reduction in the system nominal throughput.

To show the effect of using nORCs without the IC, we compare the reference scheme, single-hop (w/o-IC), to nORC (w/o-IC). We also look at the performance improvement the IC can provide by looking at nORC (w-IC). And finally, we look at the results for ORC (w-IC), to see if nORC can be used to replace ORC, especially in terms of the outage probability. The following is a summary of our observations from the performance curves,

- Relaying using nORCs is not feasible without the IC in the single-cell scenario, while provides very little improvement over single-hop (w/o-IC) in the multiplecell scenario.

- Using the IC nORC provides a significant throughput improvement over ORC, without sacrificing the outage performance of the system for single-cell and single-sector multiple-cell scenario.

- For three-sector cells, the excessive CCI introduced in the system for nORC lead to a high probability of outage, compared to ORC, which may not be justifiable by the corresponding throughput gains especially for a cluster size of one. 
- Multiple receive antennas at the mobile unit can be used to reduce the outage probability of nORC close to ORC.

Next we move to a more detailed discussion on the figures for single and multiplecell scenarios. Please note that all relaying schemes use $N_{\mathrm{R}}=6$.

\section{Single-Cell Scenario}

In the single-cell scenario, we can see that for both single and three-sector cells, single-hop (w/o-IC) outperforms nORC (w/o-IC) in terms of both throughput and outage probability. On the other hand, using the IC, nORC outperforms single-hop (w/o-IC) in both aspects as well as ORC (w-IC) in terms of throughput. Further more, the outage probability degradation compared to ORC (w-IC) is very negligible.

For example, if we consider three-sector cells, shown in Figure 4.17, we can see that $\mathrm{nORC}(\mathrm{w} / \mathrm{o}-\mathrm{IC})$ provides a throughput slightly below single-hop (w/o-IC), while nORC (w-IC) provides a throughput that is more than five times greater than singlehop (w/o-IC), which is also $75 \%$ more than the throughput provided by ORC (w-IC). This is achieved while the outage probability is significantly reduced compared to single-hop (w/o-IC), as well as being very close to the outage probability of ORC (w-IC). For example, at $R=500$ meters, we can see an outage probability close to $40 \%$ for single-hop (w/o-IC) reduced to $1.5 \%$ with nORC, while with ORC the outage probability is very close to zero. Finally if we look at Figures 4.16 and 4.17 for $N_{\mathrm{SEC}}$ $=1$ and 3 respectively, we can see that sectoring is also benificial with nORC (w-IC) in providing higher system throughput without sacrificing good outage performance. 


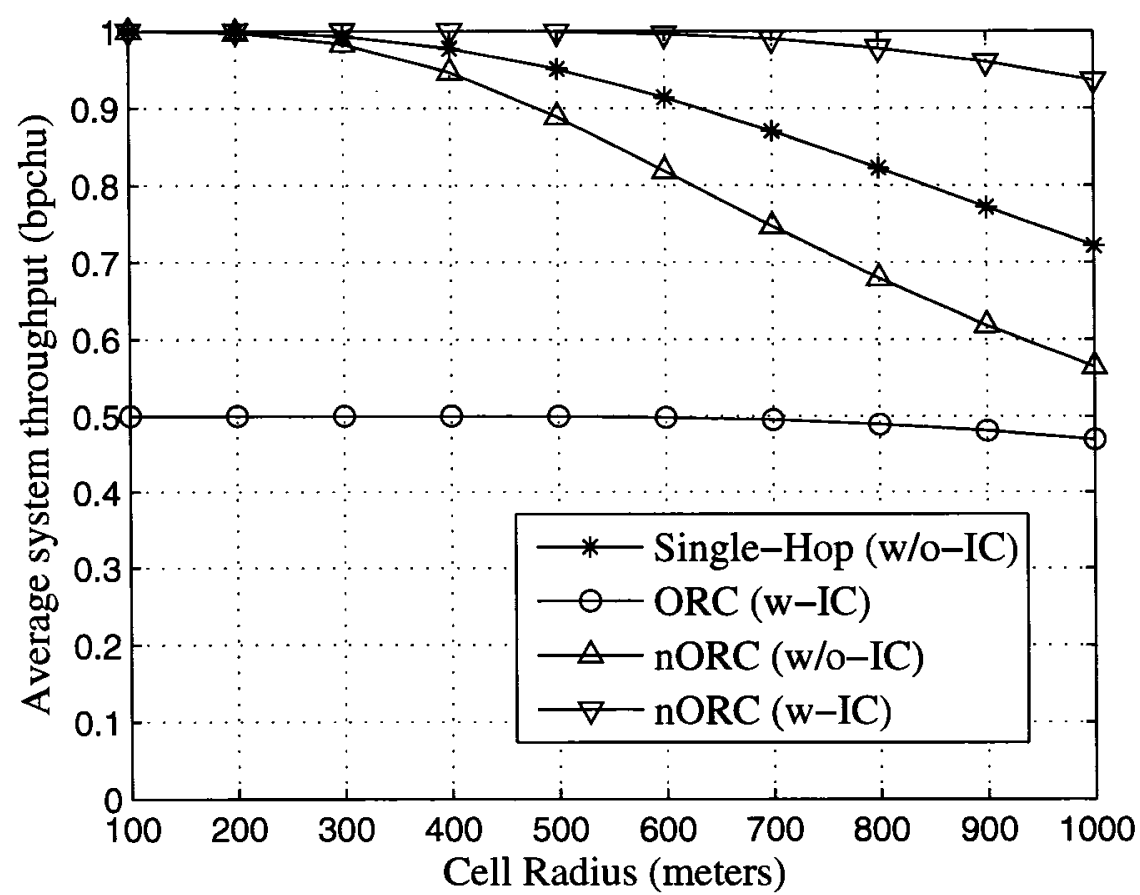

(a) Average system throughput

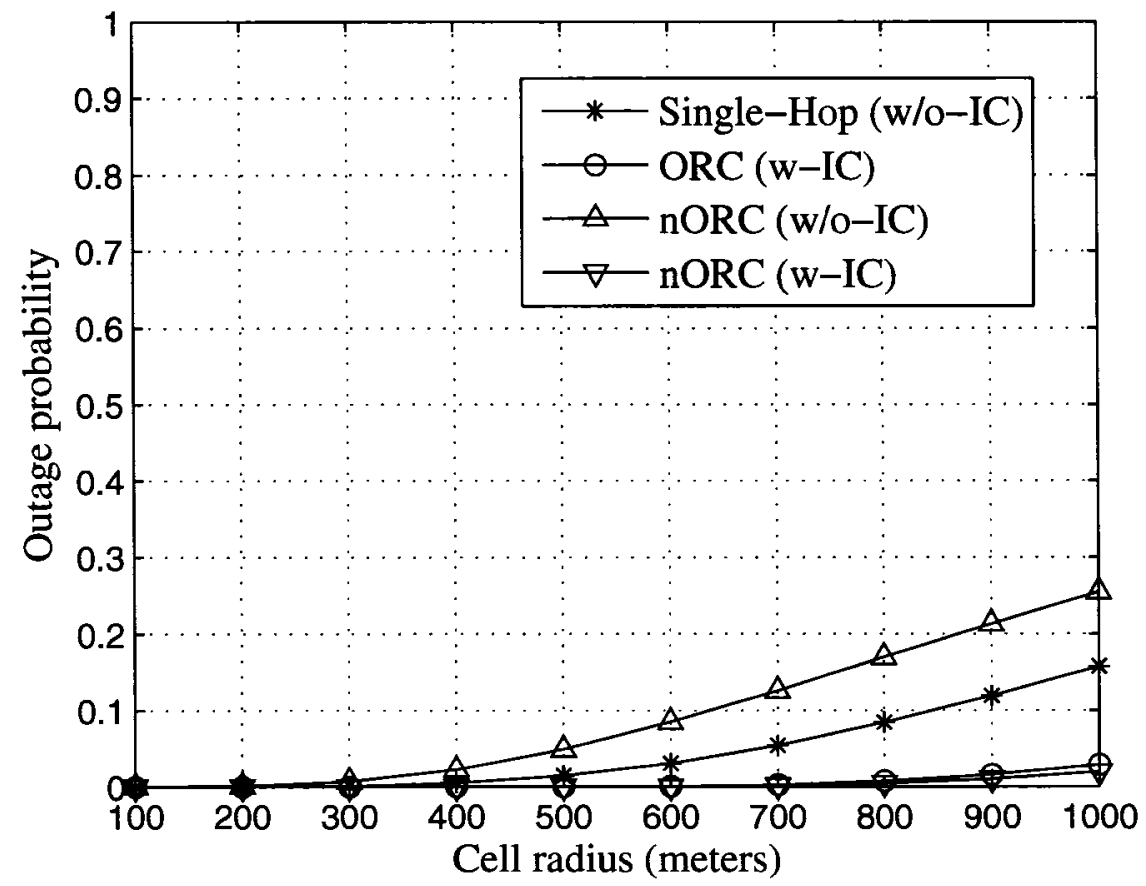

(b) Outage probability

Figure 4.16: System performance for two-hop networks using nORCs, $N_{\mathrm{CELL}}=1$ and $N_{\mathrm{SEC}}=1$. 


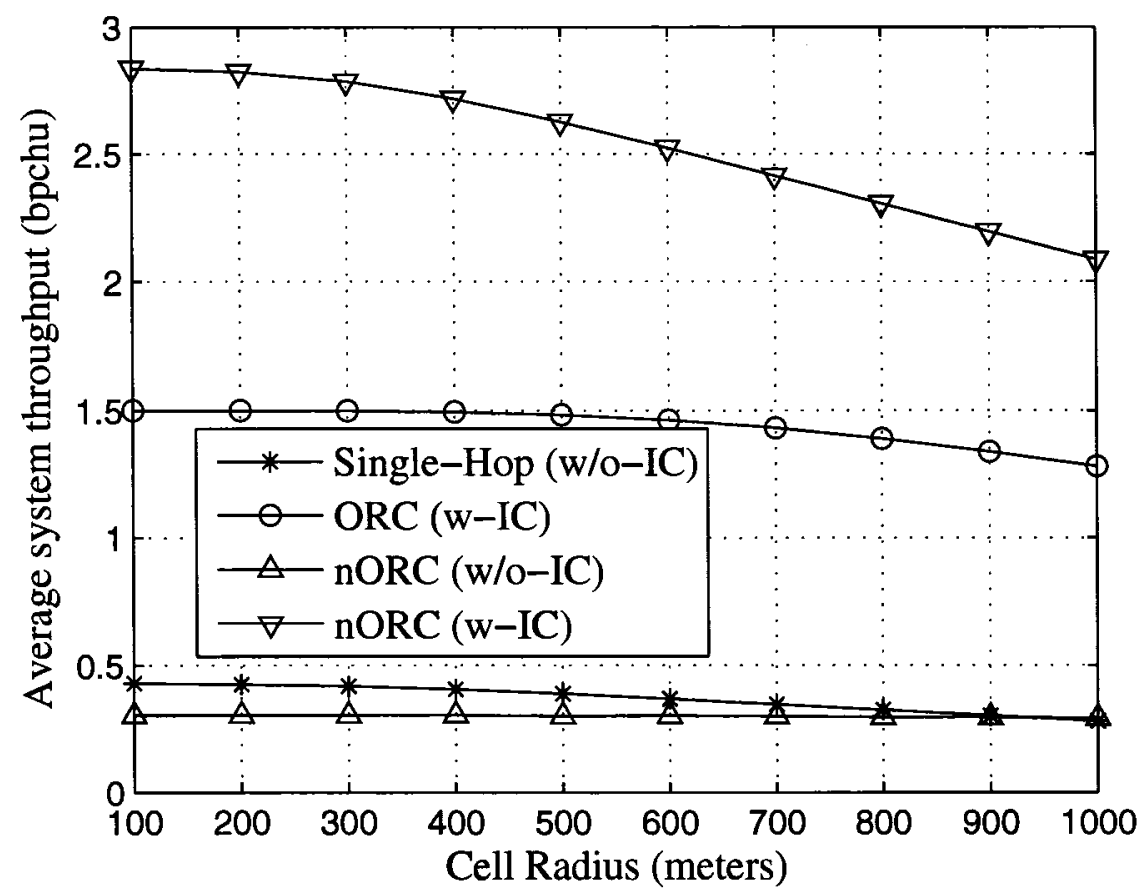

(a) Average system throughput

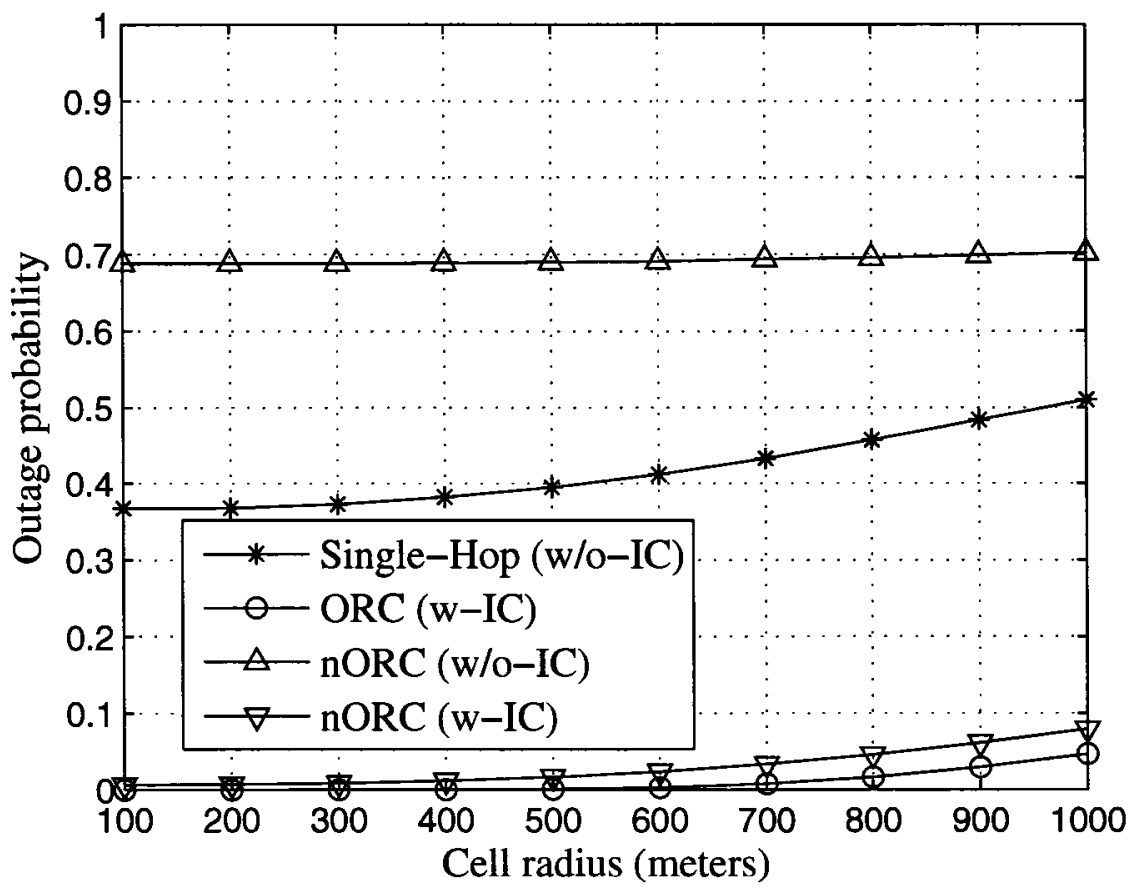

(b) Outage probability

Figure 4.17: System performance for two-hop networks using nORCs, $N_{\mathrm{CELL}}=1$ and $N_{\mathrm{SEC}}=3$. 
Multiple-Cell Scenario

In the multiple-cell scenario, the additional CCI from all surrounding cells makes using nORCs a lot harder. Of course, without the IC the throughput and outage performance of nORC is very close to single-hop (w/o-IC) which is very poor. Cluster size of one, shown in Figures 4.18 for $N_{\mathrm{SEC}}=1$ and 4.19 for $N_{\mathrm{SEC}}=1$, has the most severe CCI, that is why the throughput improvement that $\mathrm{nORC}$ achieves may not justify the significant increase in outage probability that accompanies it, especially for $N_{\mathrm{SEC}}=3$, where nORC (w-IC) provides slightly improves throughput compared to ORC (w-IC) while the outage probability is more than doubled. For $N_{\mathrm{SEC}}=$ 1 , the situation is not that bad, however nORC still suffers from a poor outage performance. In Figures 4.20 and 4.21 we look at the range of improvement for two receive antennas at the receiver for the same cluster size. For $N_{\mathrm{SEC}}=1$, nORC (PIC3) provides a 70\% increase in throughput over ORC (PIC3), while the outage probability is below $10 \%$. Note that nORC (PIC1) has a very close performance to nORC (w-IC), which indicates a promising actual performance close to nORC (PIC3) for MUs with two receive antennas. Unfortunately for $N_{\mathrm{SEC}}=3$, even for nORC (PIC3), outage remains at a very high level, which indicates that relaying on nORCs may be hard in this particular cellular layout due to the excessive CCI that exists.

Next we look at cluster size of four, shown in Figures 4.22 for $N_{\mathrm{SEC}}=1$ and 4.23 for $N_{\mathrm{SEC}}=1$, where CCI is less severe. For $N_{\mathrm{SEC}}=1, \mathrm{nORC}(\mathrm{w}-\mathrm{IC})$ provides an $80 \%$ increase in throughput over ORC (w-IC), with a very good outage performance that even outperforms ORC (w-IC) at large cell sizes. This is because CCI decreases in larger cell sizes and hence ORC (w-IC) can not provide enough probability of error reduction to compensate for the $50 \%$ loss in nominal throughput, which does not 
exist in the cae of nORC. In the case of three-sector cells, $N_{\mathrm{SEC}}=3$, the outage probability of nORC (w-IC) is around $20 \%$ which may be considered high regardless of the $30 \%$ increase in throughput over ORC (w-IC). However, as shown in Figure 4.24 , the outage probability for $\mathrm{nORC}$ (PIC3) is less than $5 \%$ and is very close to ORC (PIC3), while the throughput of ORC (PIC3) is less than that of both nORC (w-IC) and nORC (PIC3). Therefore, in general we can see that for $C_{\mathrm{S}}=4$, nORC (w-IC) can be used instead of ORC (w-IC) in order to provide more significant throughput improvements while maintaining good outage performance especially with MUs having more than one receive antenna. 


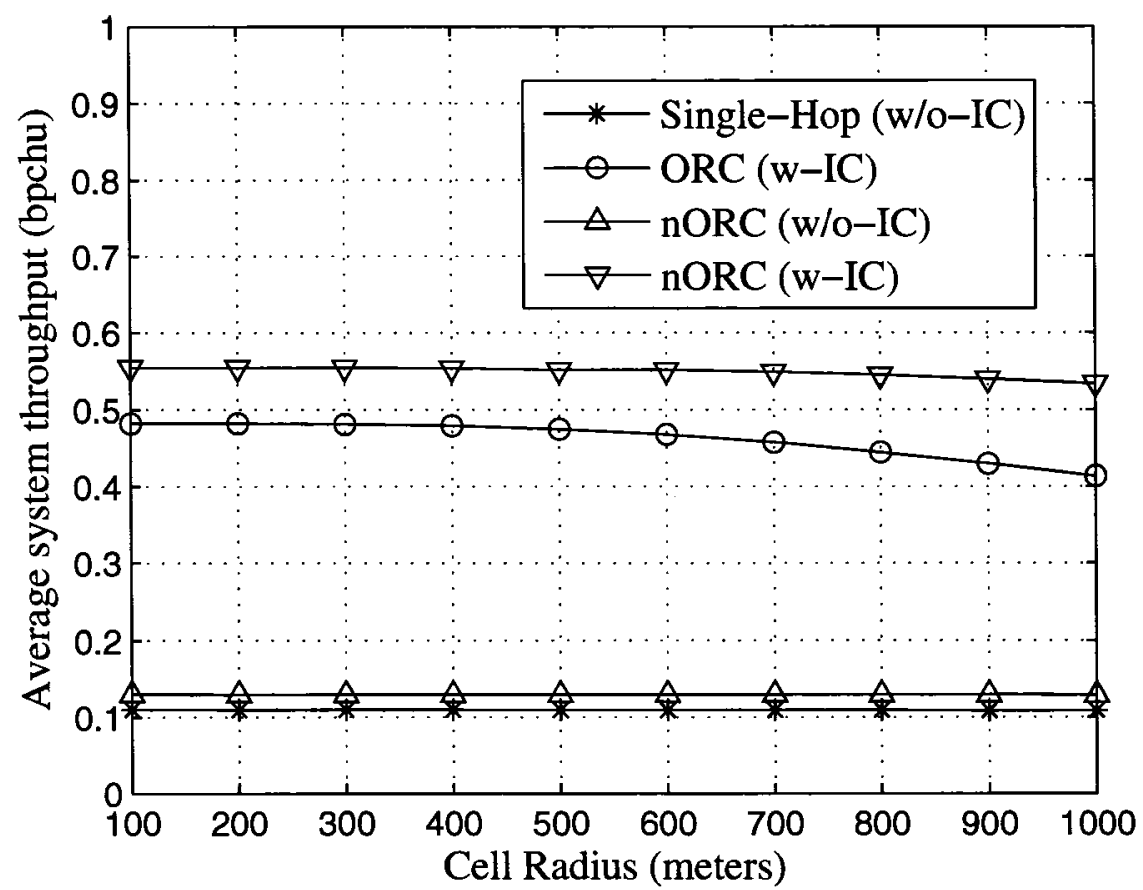

(a) Average system throughput

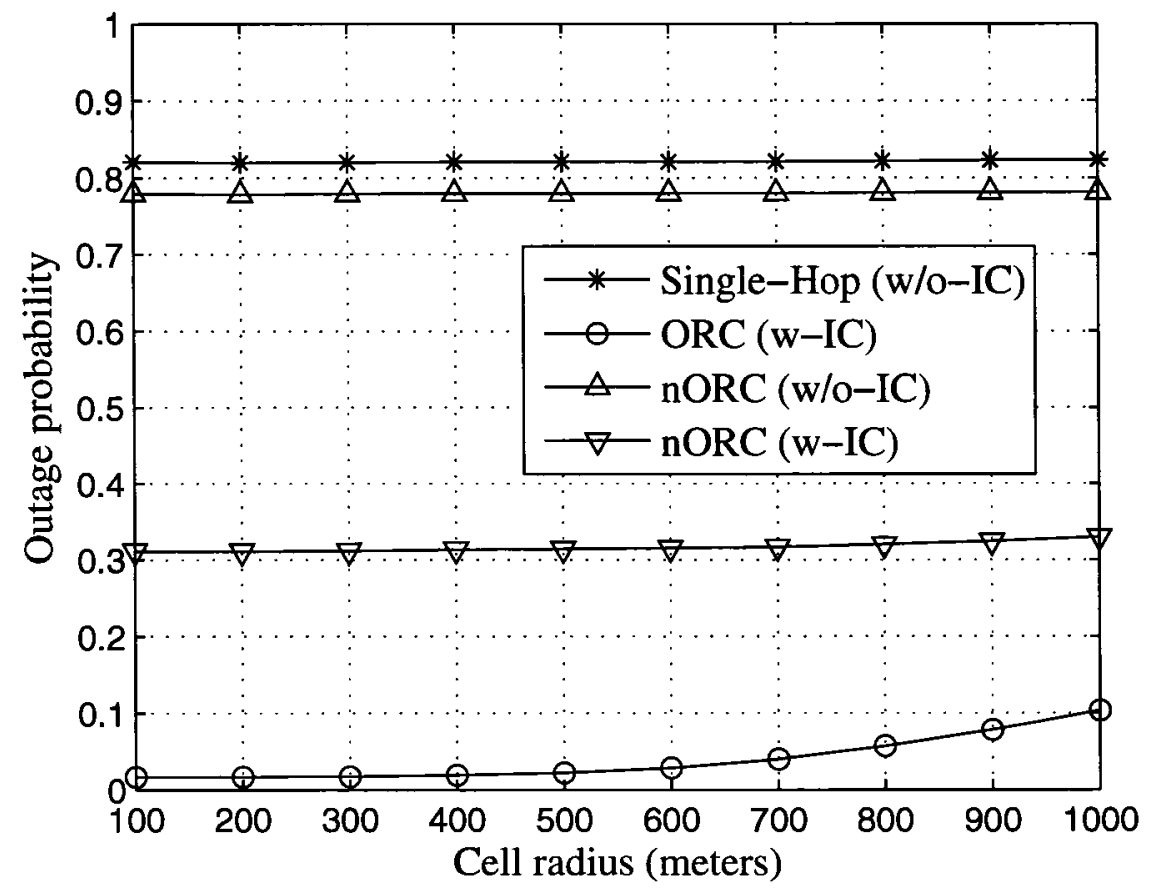

(b) Outage probability

Figure 4.18: System performance for two-hop networks using nORCs, $N_{\text {CELL }}=7$, $N_{\mathrm{SEC}}=1$, and $C_{\mathrm{S}}=1$. 


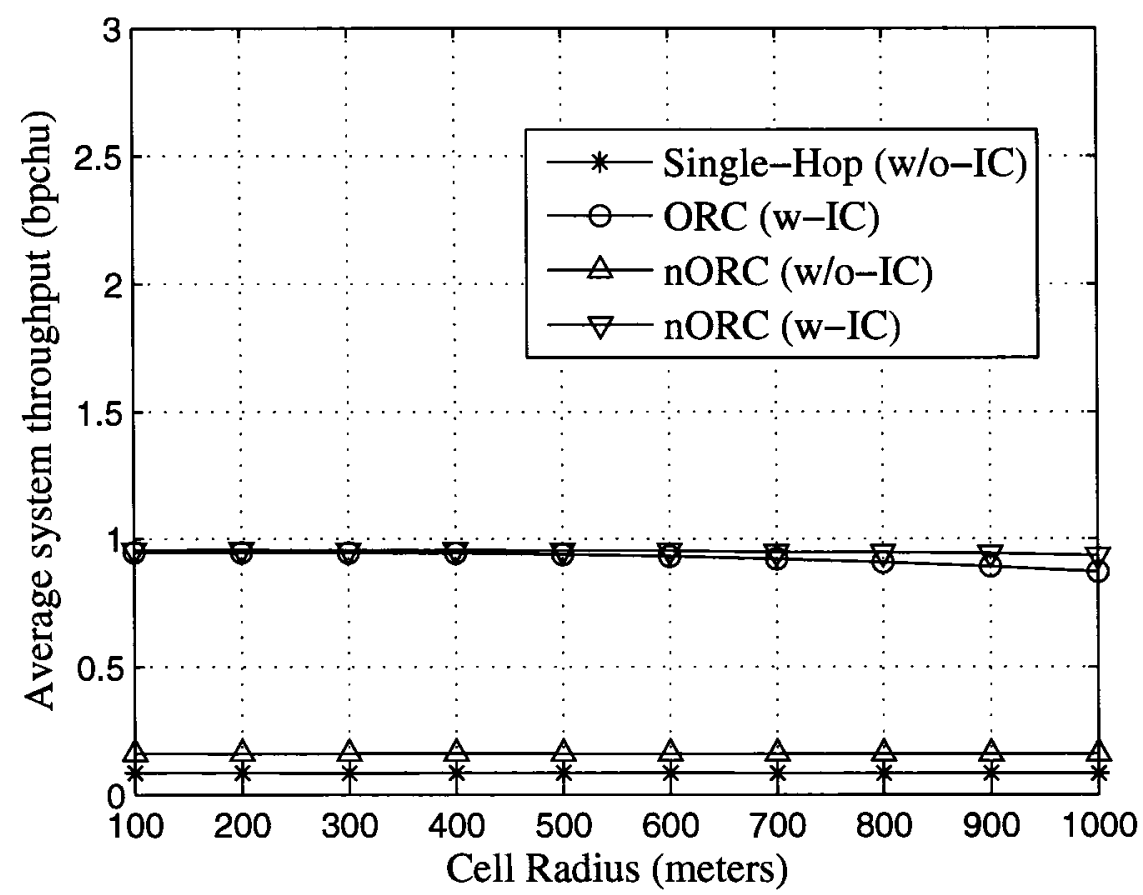

(a) Average system throughput

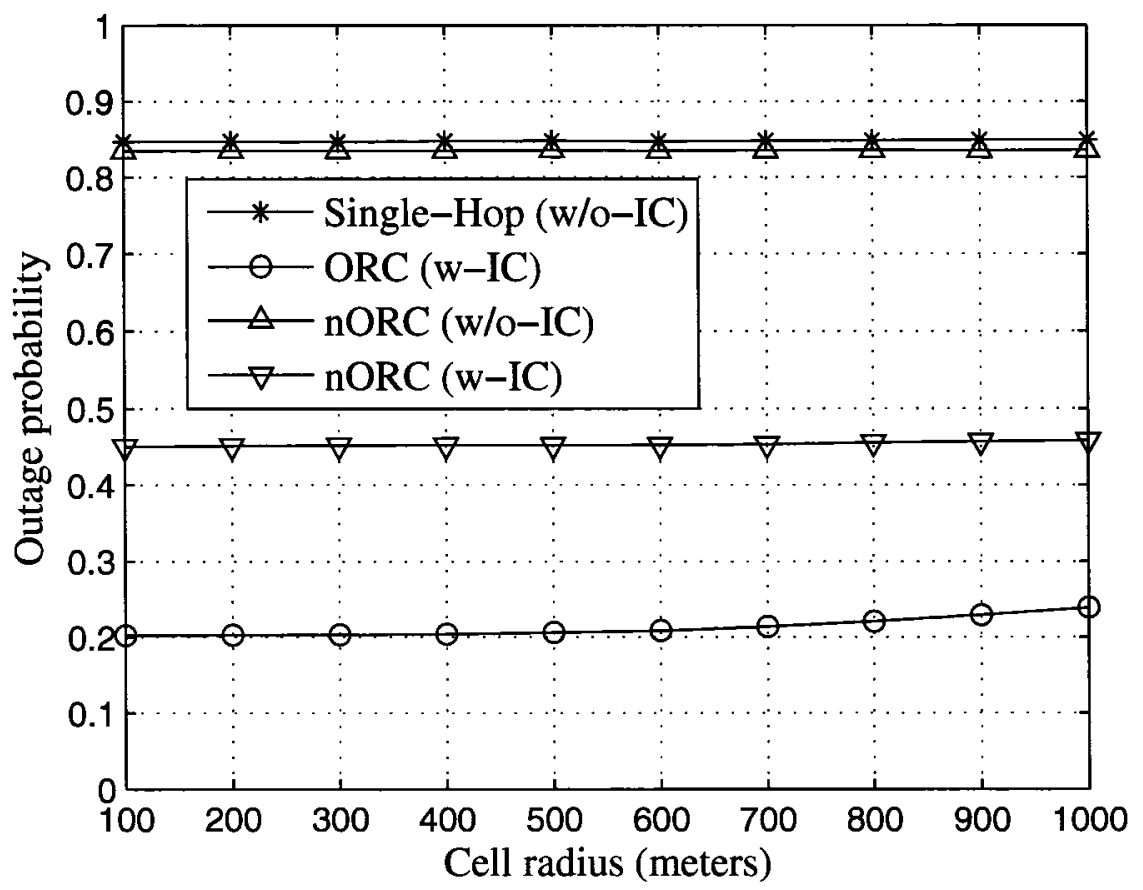

(b) Outage probability

Figure 4.19: System performance for two-hop networks using nORCs, $N_{\mathrm{CELL}}=7$, $N_{\mathrm{SEC}}=3$, and $C_{\mathrm{S}}=1$. 


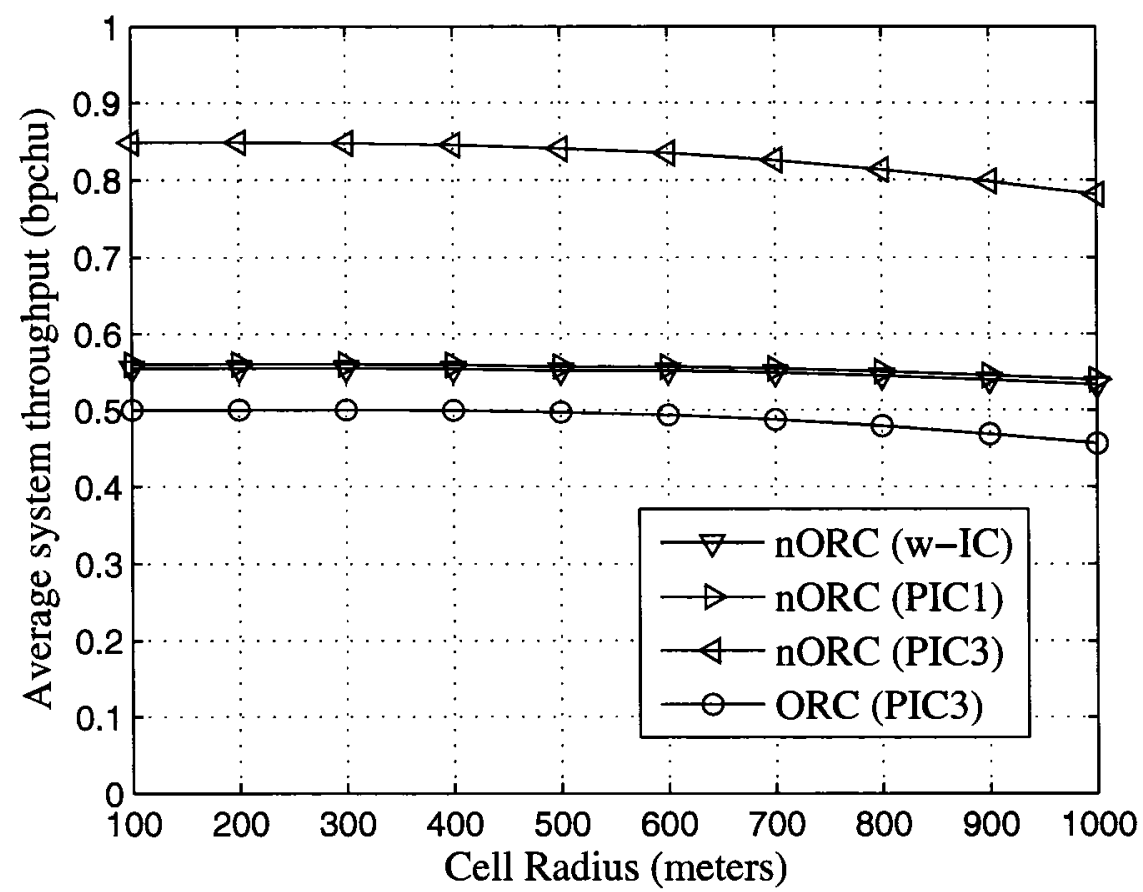

(a) Average system throughput

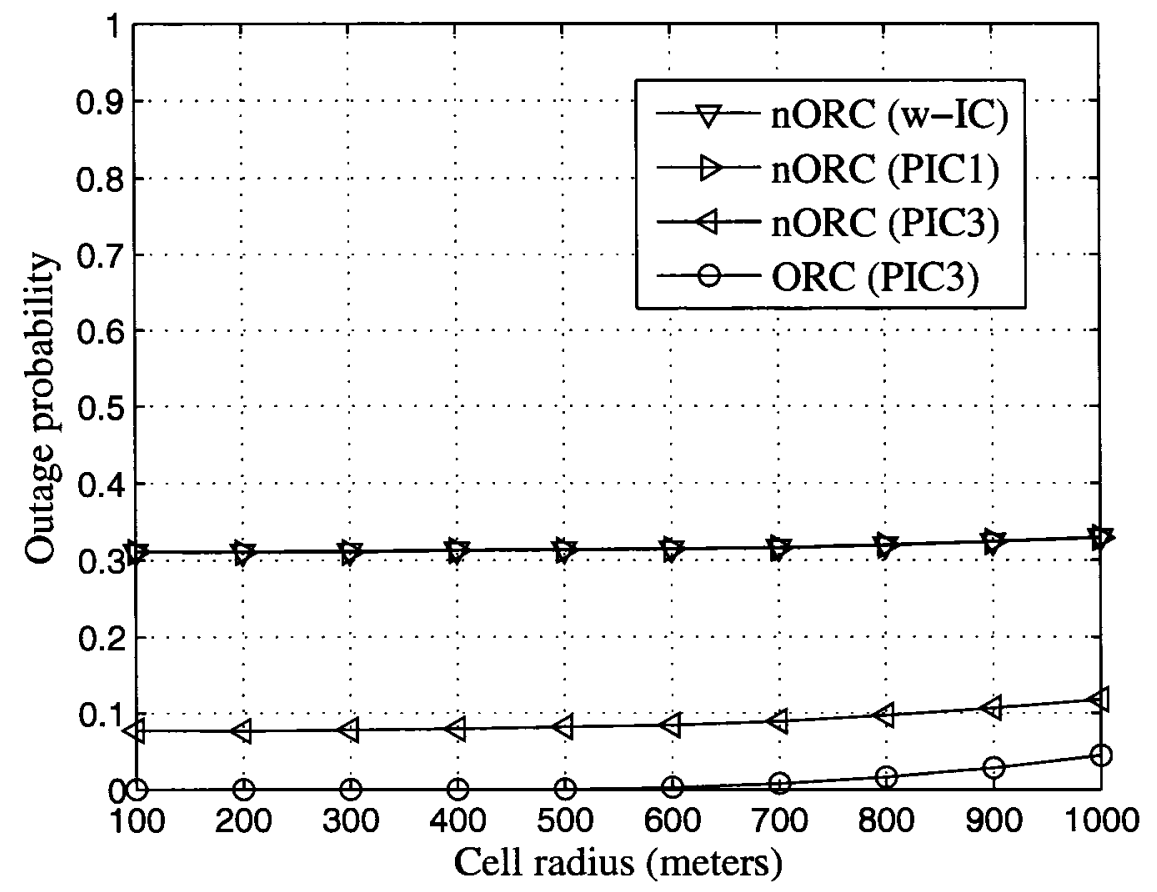

(b) Outage probability

Figure 4.20: System performance with perfect interference cancellation (PIC) for twohop networks using nORCs, $N_{\mathrm{CELL}}=7, N_{\mathrm{SEC}}=1$, and $C_{\mathrm{S}}=1$. 


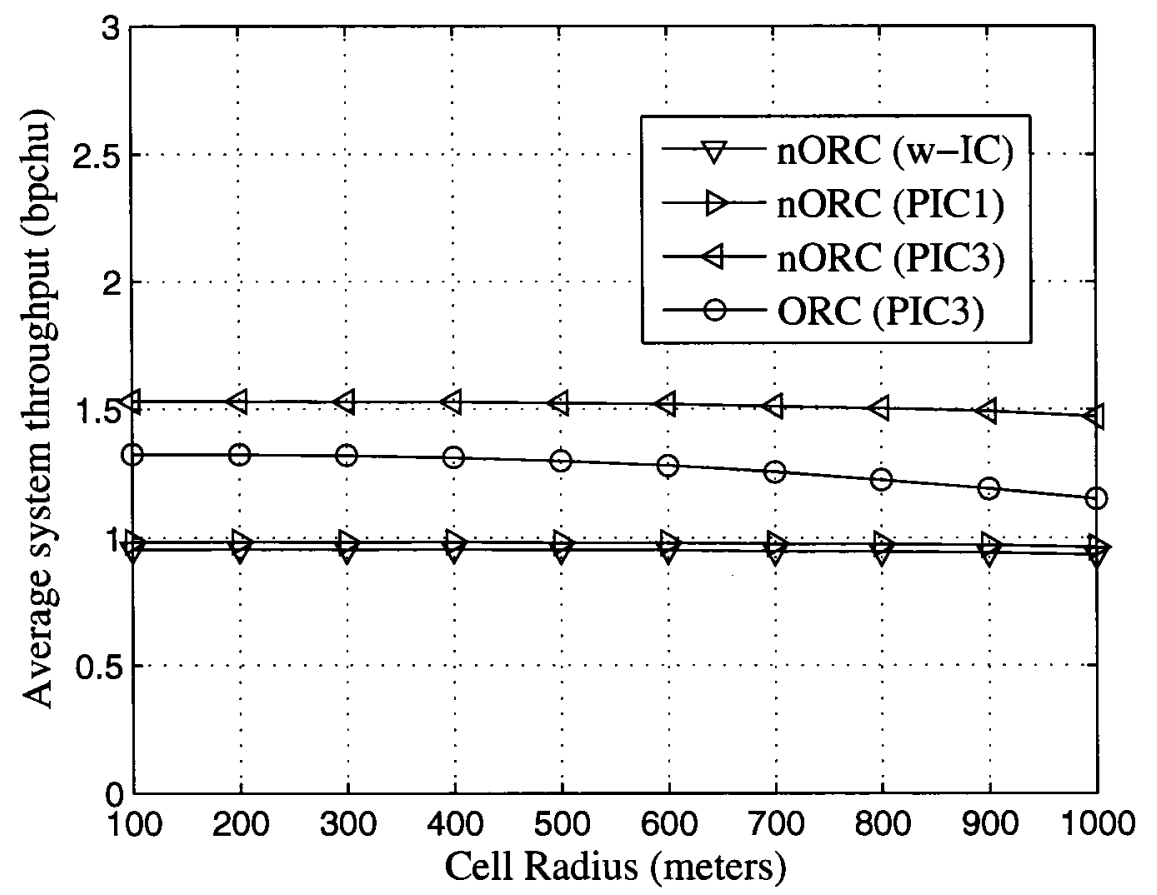

(a) Average system throughput

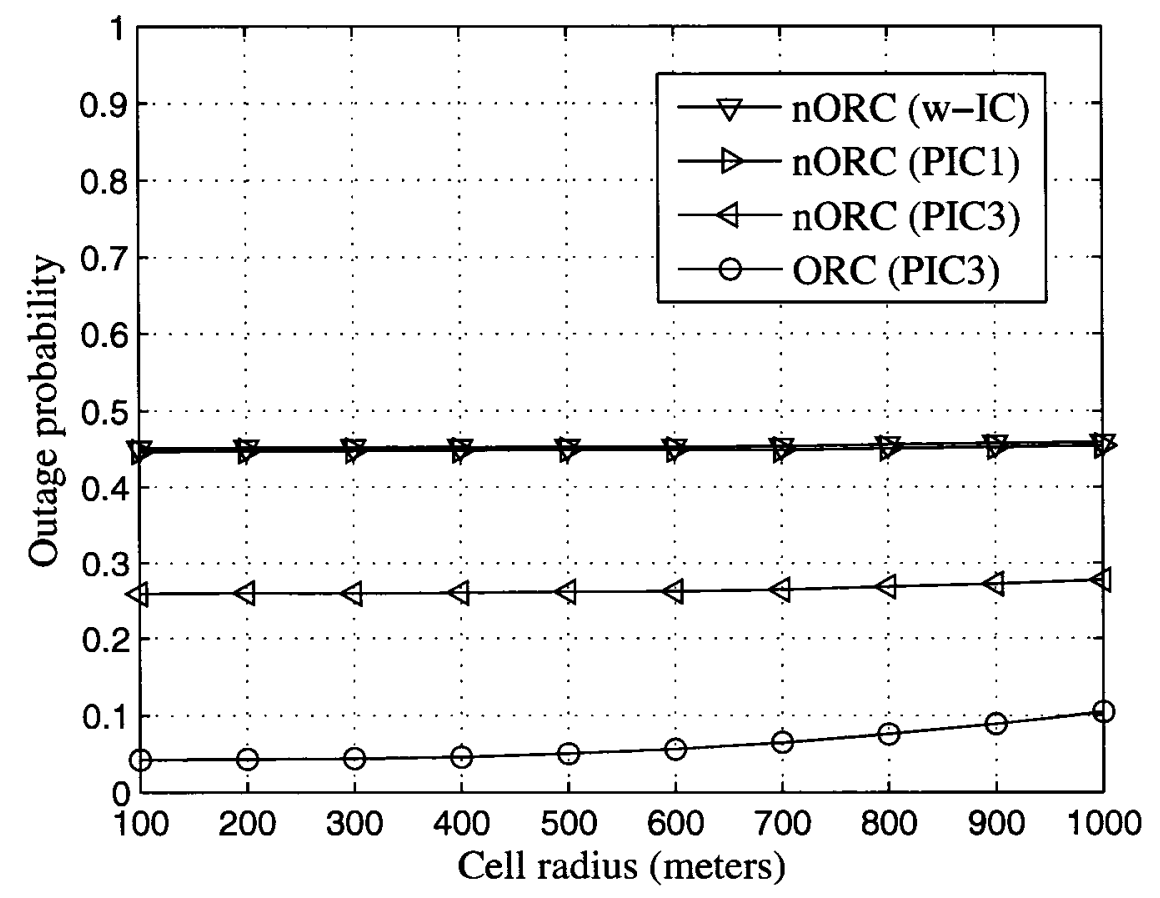

(b) Outage probability

Figure 4.21: System performance with perfect interference cancellation (PIC) for twohop networks using nORCs, $N_{\mathrm{CELL}}=7, N_{\mathrm{SEC}}=3$, and $C_{\mathrm{S}}=1$. 


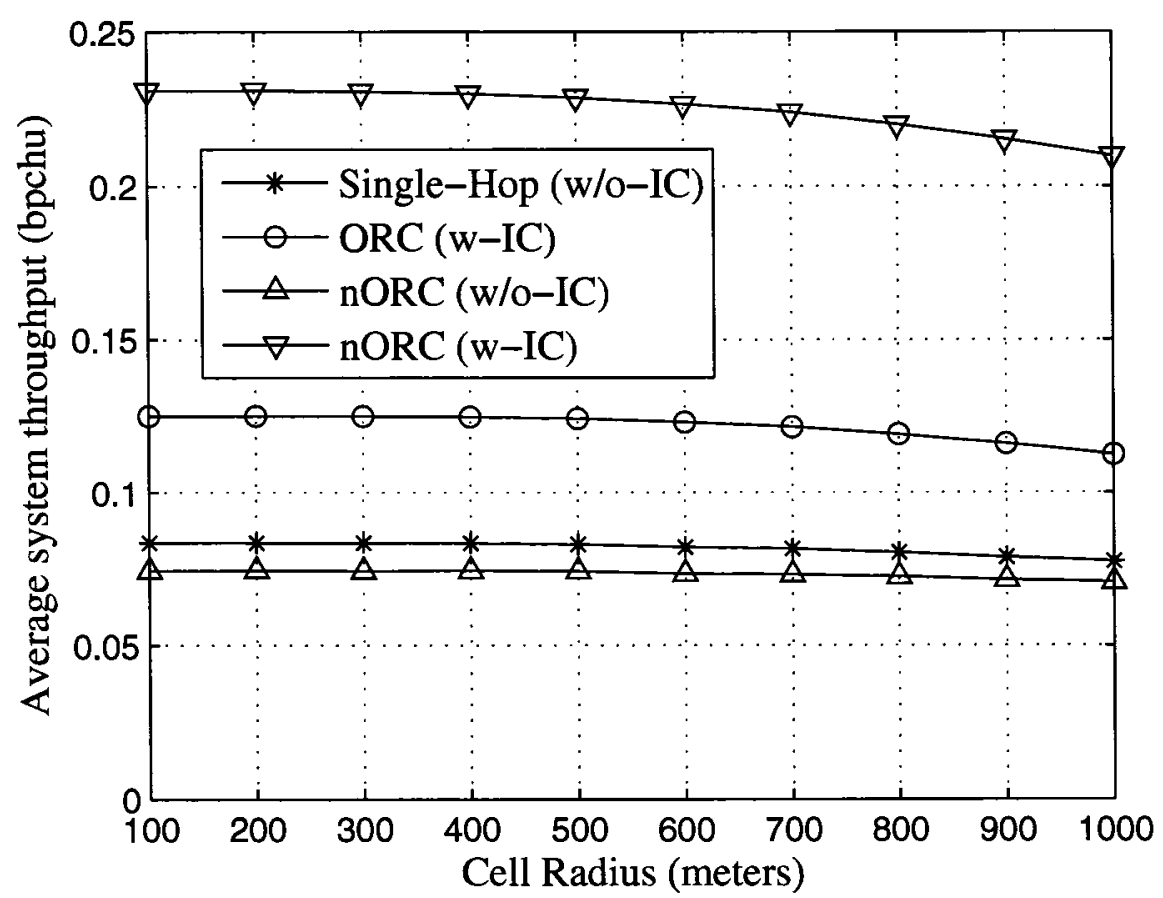

(a) Average system throughput

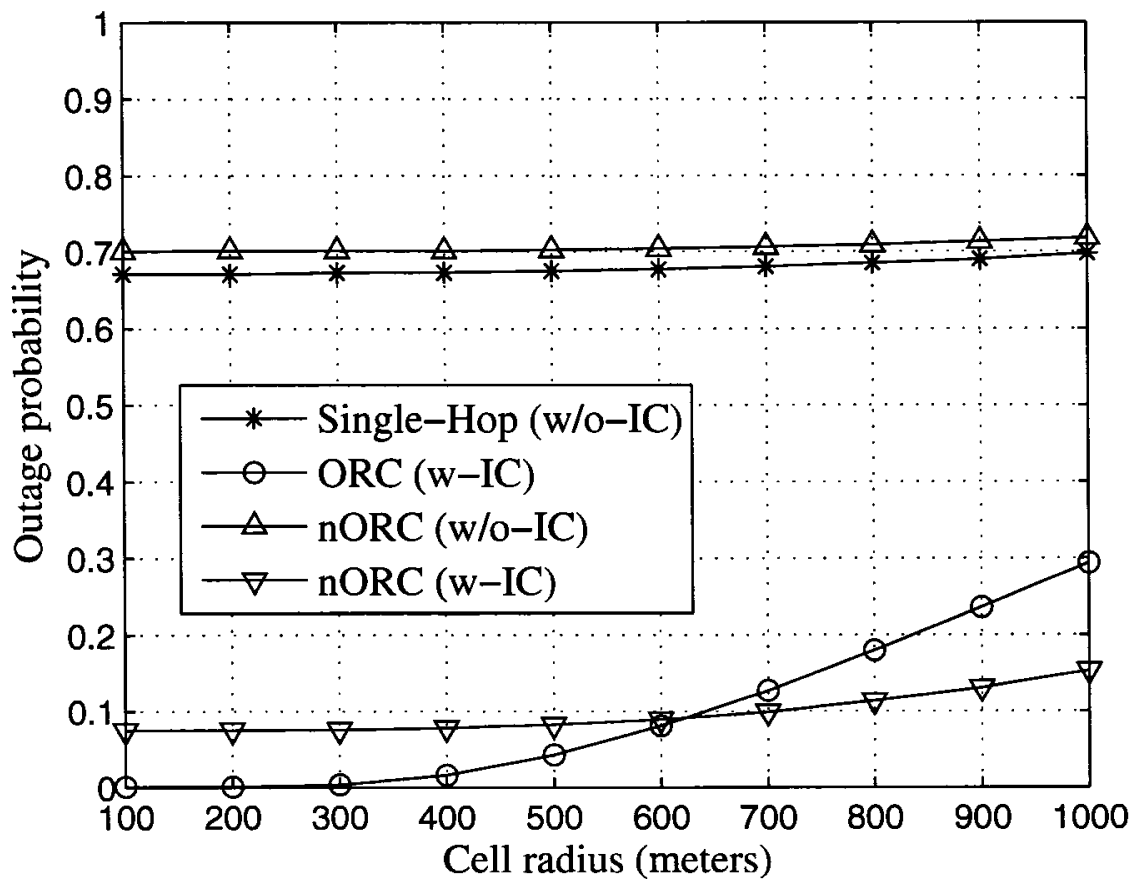

(b) Outage probability

Figure 4.22: System performance for two-hop networks using nORCs, $N_{\mathrm{CELL}}=7$, $N_{\mathrm{SEC}}=1$, and $C_{\mathrm{S}}=4$. 


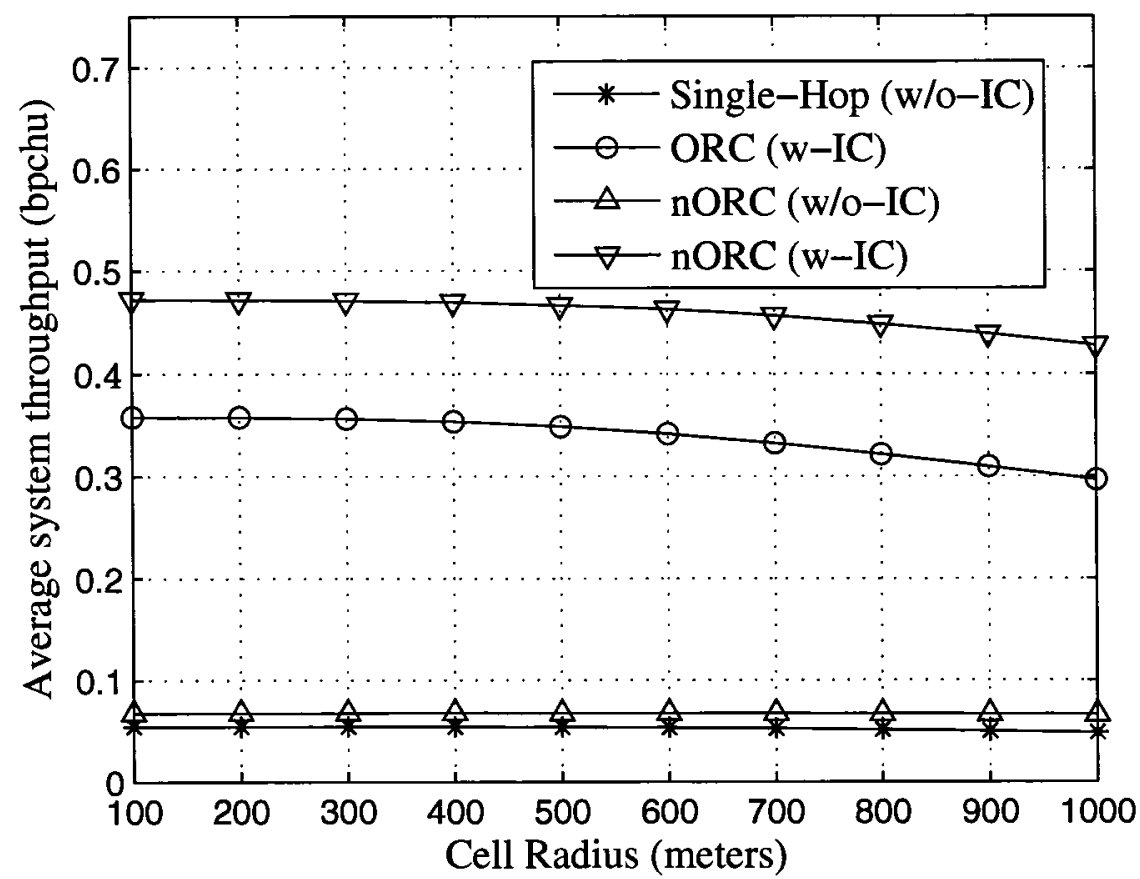

(a) Average system throughput

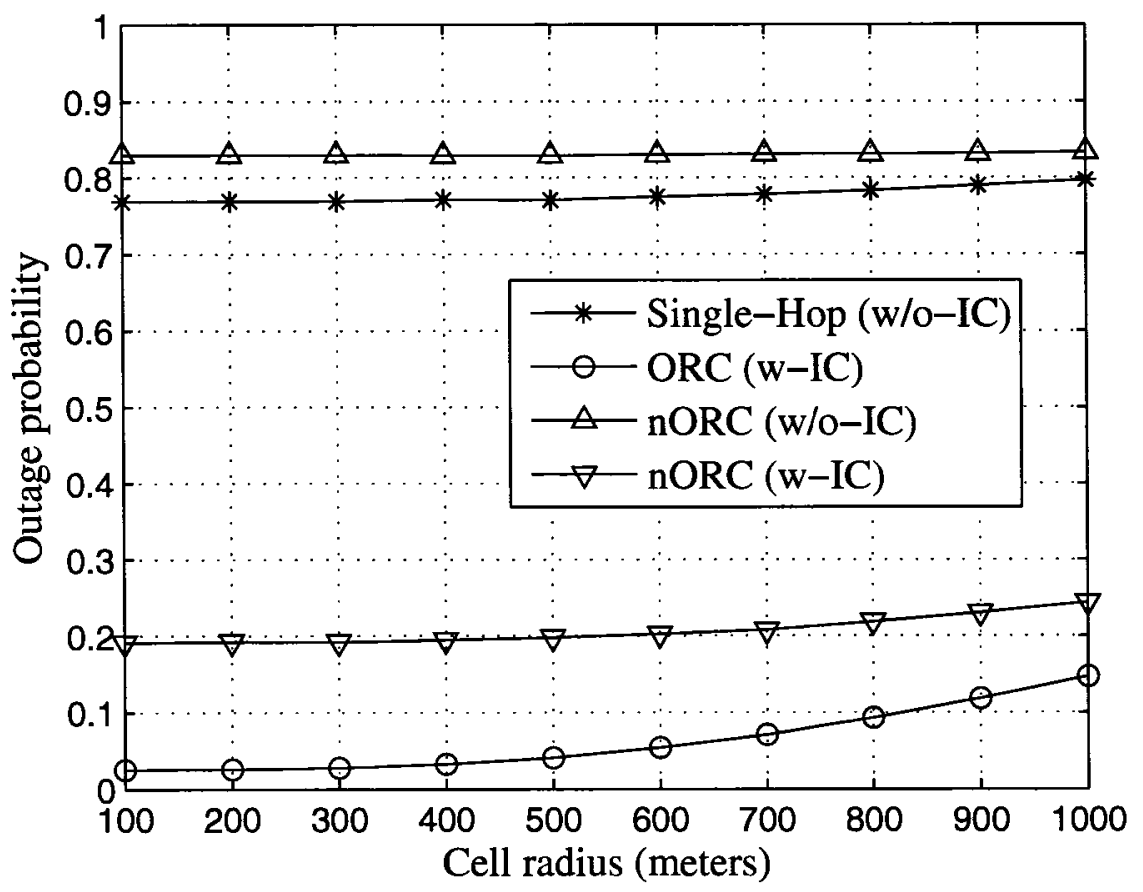

(b) Outage probability

Figure 4.23: System performance for two-hop networks using nORCs, $N_{\text {CELL }}=7$, $N_{\mathrm{SEC}}=3$, and $C_{\mathrm{S}}=4$. 


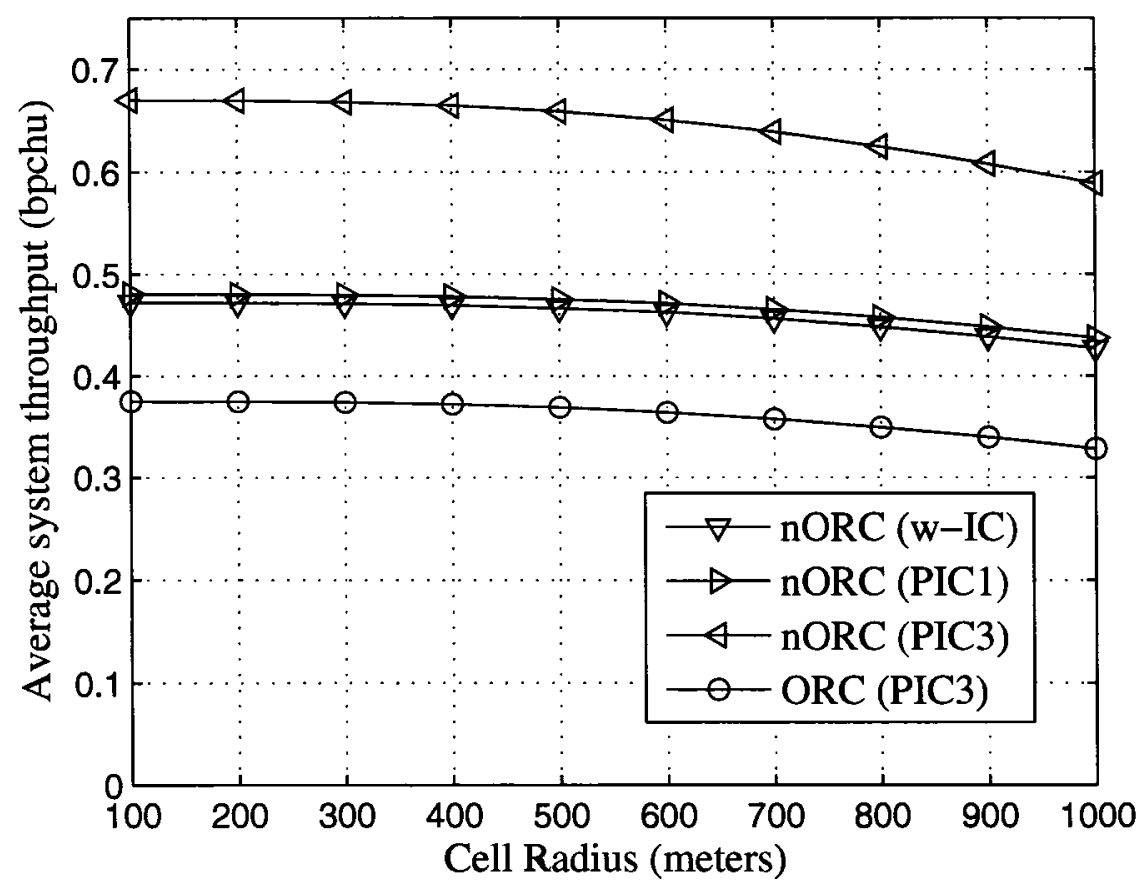

(a) Average system throughput

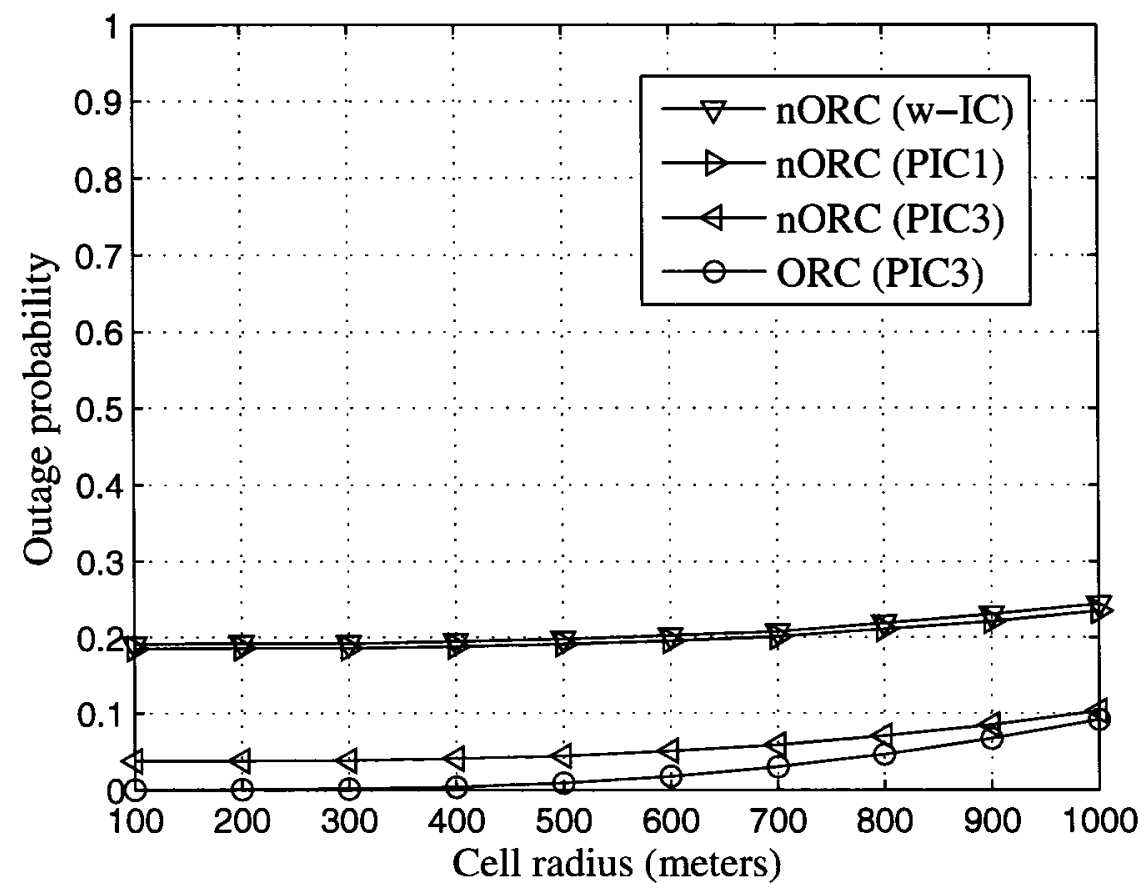

(b) Outage probability

Figure 4.24: System performance with perfect interference cancellation (PIC) for twohop networks using nORCs, $N_{\mathrm{CELL}}=7, N_{\mathrm{SEC}}=3$, and $C_{\mathrm{S}}=4$. 


\subsubsection{The Effect of the IC on Relaying Benefits}

In the previous two sections, we always compared the different relaying schemes, whether ORC or nORC, with single-hop (w/o-IC). In this section we compare singlehop (w-IC) to ORC and nORC (w-IC), to investigate the effect of the IC on the effectiveness and the benefits of relaying. Below is a summary of our observations on the performance curves shown in this section

- Relaying using nORCs outperforms single-hop (w-IC) in all scenarios in terms of both system throughput and probability of outage.

- For single-cell applications single-hop (w-IC) provides much better throughput performance with a small penalty in terms of outage probability compared to ORC (w-IC), especially for small and medium size cells.

- For a cluster size of one multiple-cell scenario, single-hop (w-IC) suffers from a very poor outage performance as well as low system throughput that is even worst than ORC (w-IC).

- For a cluster size of four, single-hop (w-IC) provides good system throughput higher than the half system nominal throughput ceiling that ORC suffers from, however this is accompanied with an increase in outage probability that can be mitigated through the use of multiple receive antennas, as it is the case with nORC at the same cluster size.

\section{Single-Cell Scenario}

For a single-cell and $N_{\mathrm{SEC}}=1$, single-hop (w-IC) is equivalent to the (w/o-IC) case which has been shown before. So, our discussion for the single-cell scenario is 
limited to $N_{\mathrm{SEC}}=3$ case shown in Figure 4.25. As seen in the figure nORC (wIC) provides better throughput and outage results than single-hop (w-IC) especially as the cell size increases. On the other hand, we can see that single-hop (w-IC) provides much better throughput performance than ORC (w-IC) with a low outage probability, at least up to $R=600$ meters, where the average throughput is close to 2.5 times that of ORC (w-IC) while the system outage is below $5 \%$. 


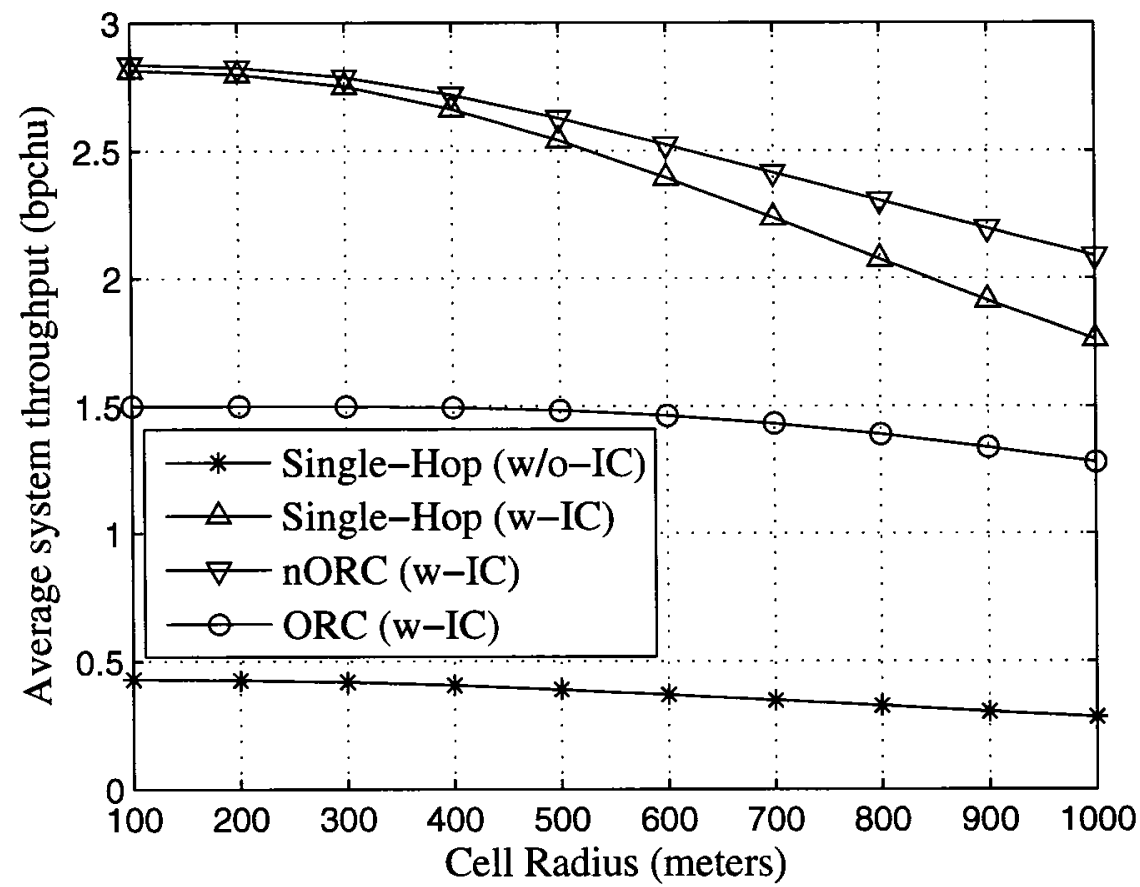

(a) Average system throughput

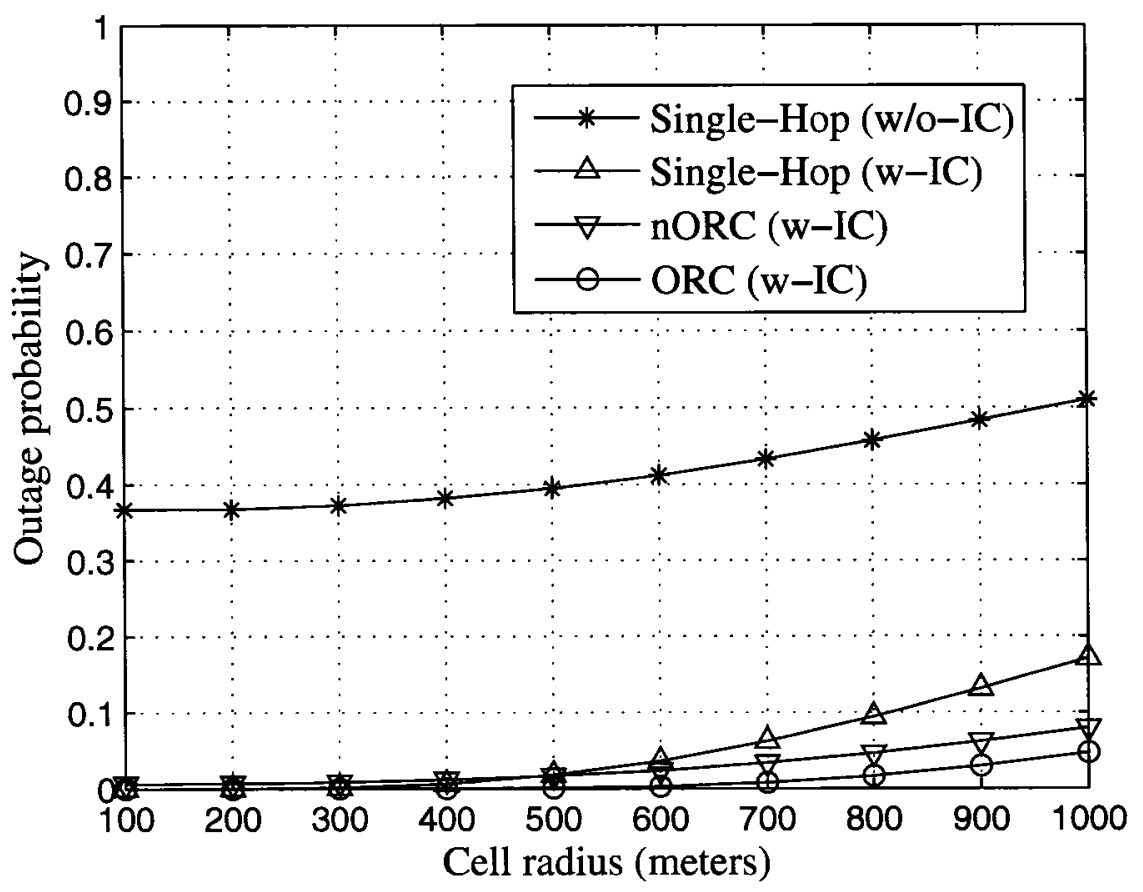

(b) Outage probability

Figure 4.25: System performance using IC, single vs. two-hop networks for $N_{\text {CELL }}=1$ and $N_{\mathrm{SEC}}=3$. 


\section{Multiple-Cell Scenario}

The results for cluster size of one are shown in Figures 4.26 and 4.27 for $N_{\mathrm{SEC}}$ $=1$ and 3 respectively. We can see that for both cases, single-hop (w-IC) has a lower throughput than both ORC and nORC (w-IC), together with a very high outage probability, which indicates that in such layouts relaying, and maybe more specifically using ORCs, would be essential to achieve a descent system performance especially in terms of outage probability.

For cluster size of four, shown in Figures 4.28 and 4.29 for $N_{\mathrm{SEC}}=1$ and 3 respectively, single-hop (w-IC) provides a system throughput that is higher than half the system nominal throughput, and hence it always outperforms ORC (w-IC) in that aspect. Furthermore, the outage probability of single-hop (w-IC) is not significantly higher than ORC (w-IC), and hence is expected to be reduced further for MUs having multiple receive antennas while providing even better throughput results. 


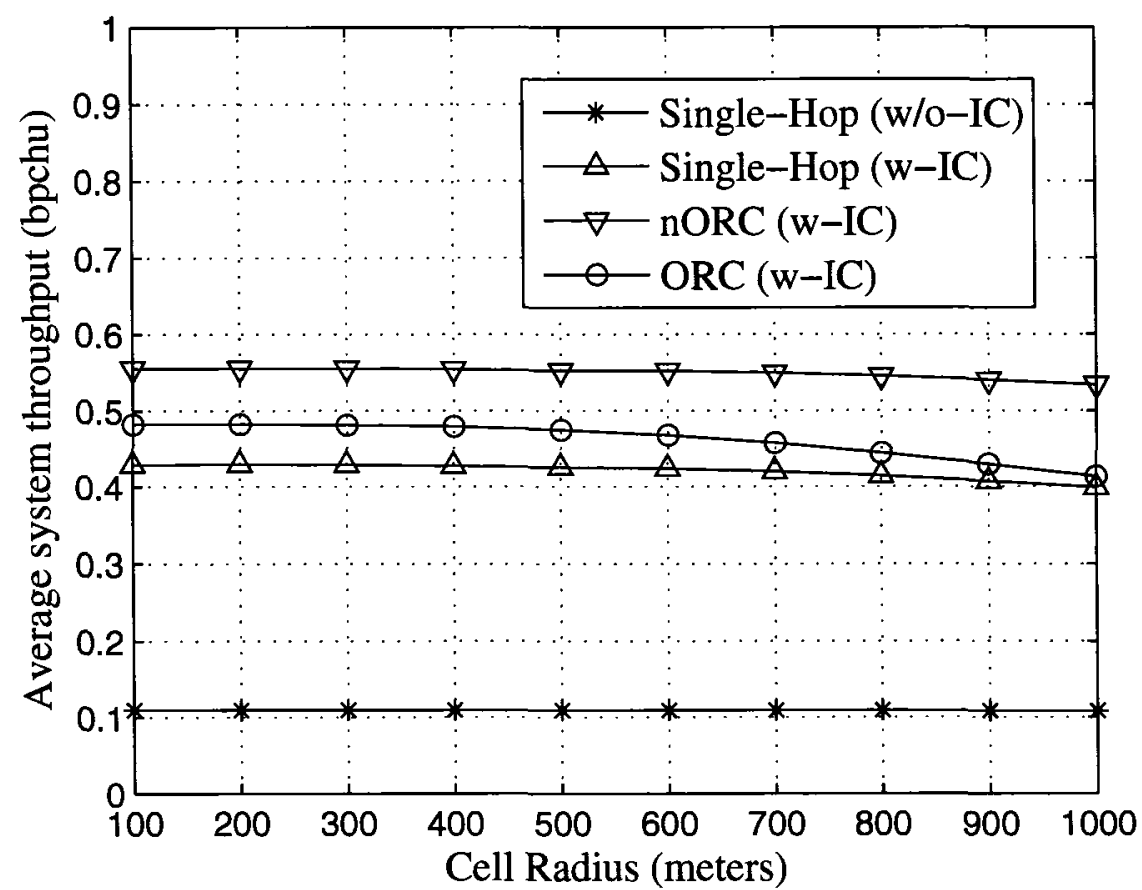

(a) Average system throughput

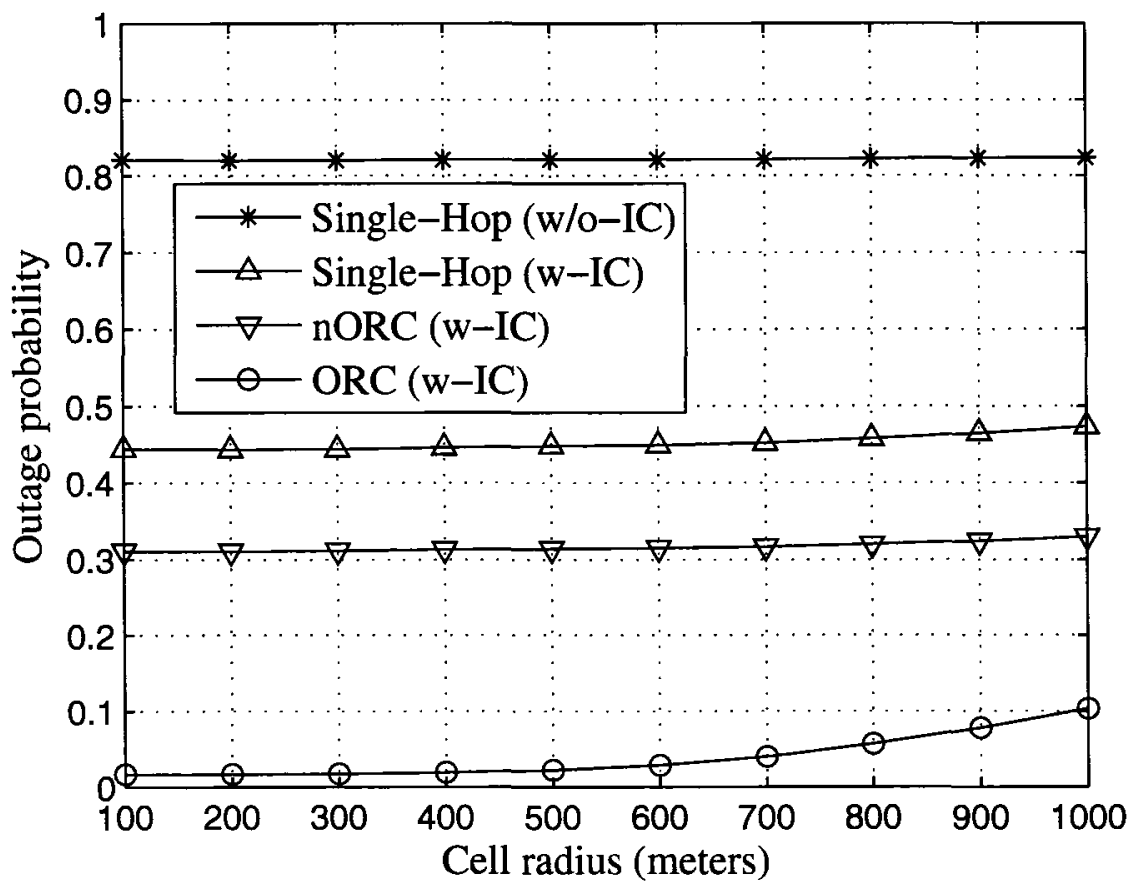

(b) Outage probability

Figure 4.26: System performance using IC, single vs. two-hop networks for $N_{\text {CELL }}=7$, $N_{\mathrm{SEC}}=1$, and $C_{\mathrm{S}}=1$. 


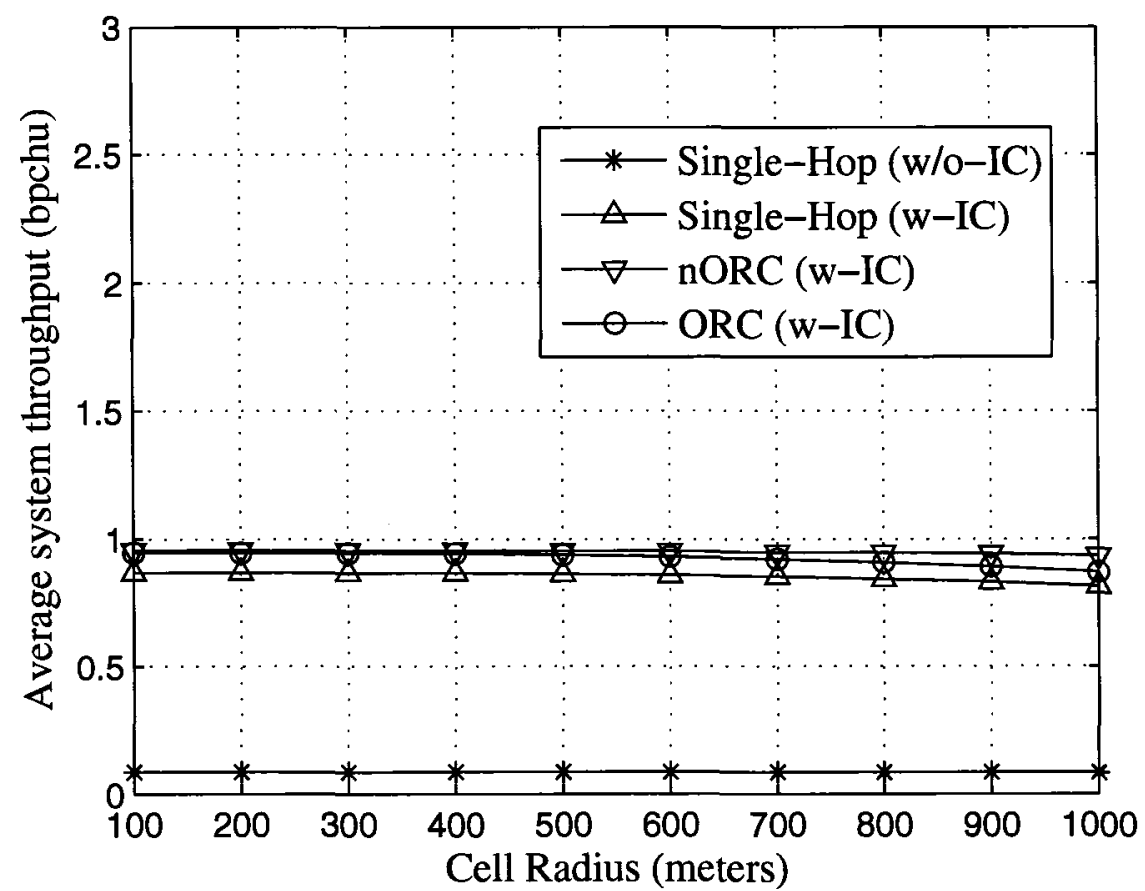

(a) Average system throughput

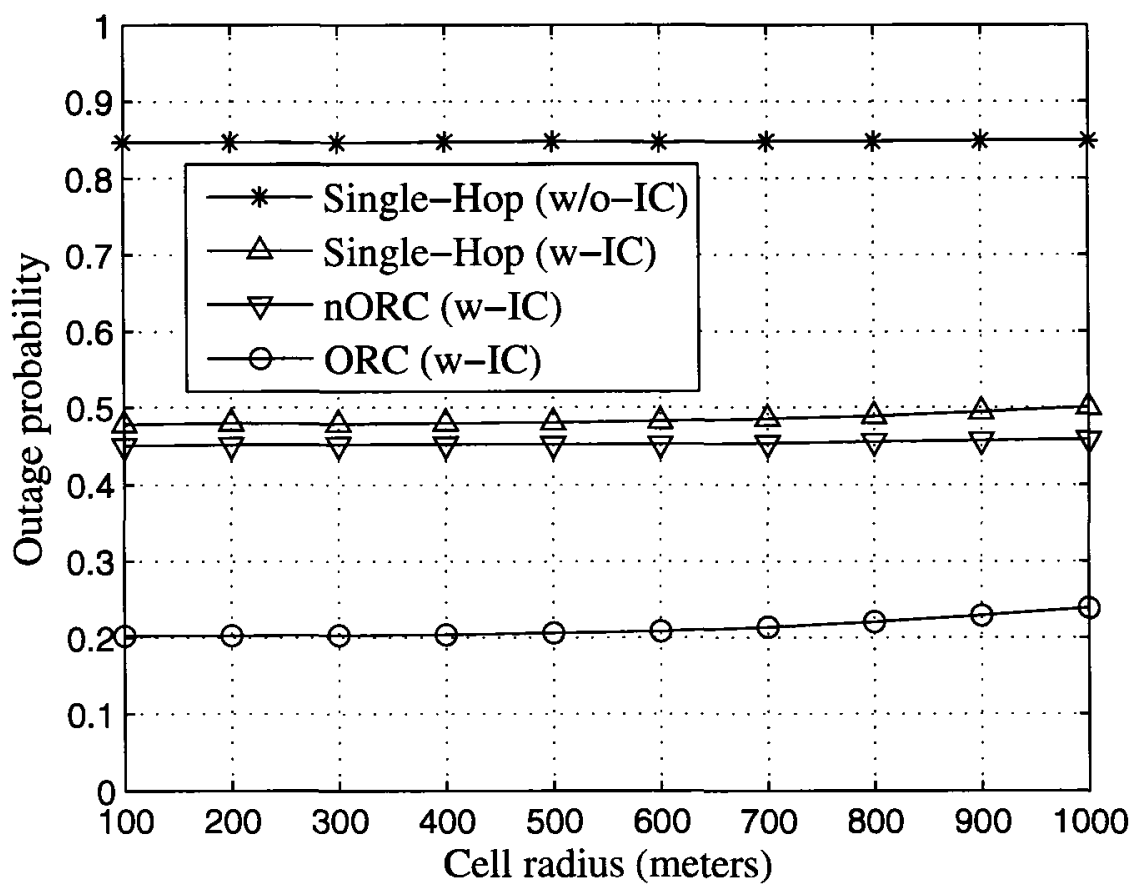

(b) Outage probability

Figure 4.27: System performance using IC, single vs. two-hop networks for $N_{\text {CELL }}=7$, $N_{\mathrm{SEC}}=3$, and $C_{\mathrm{S}}=1$. 


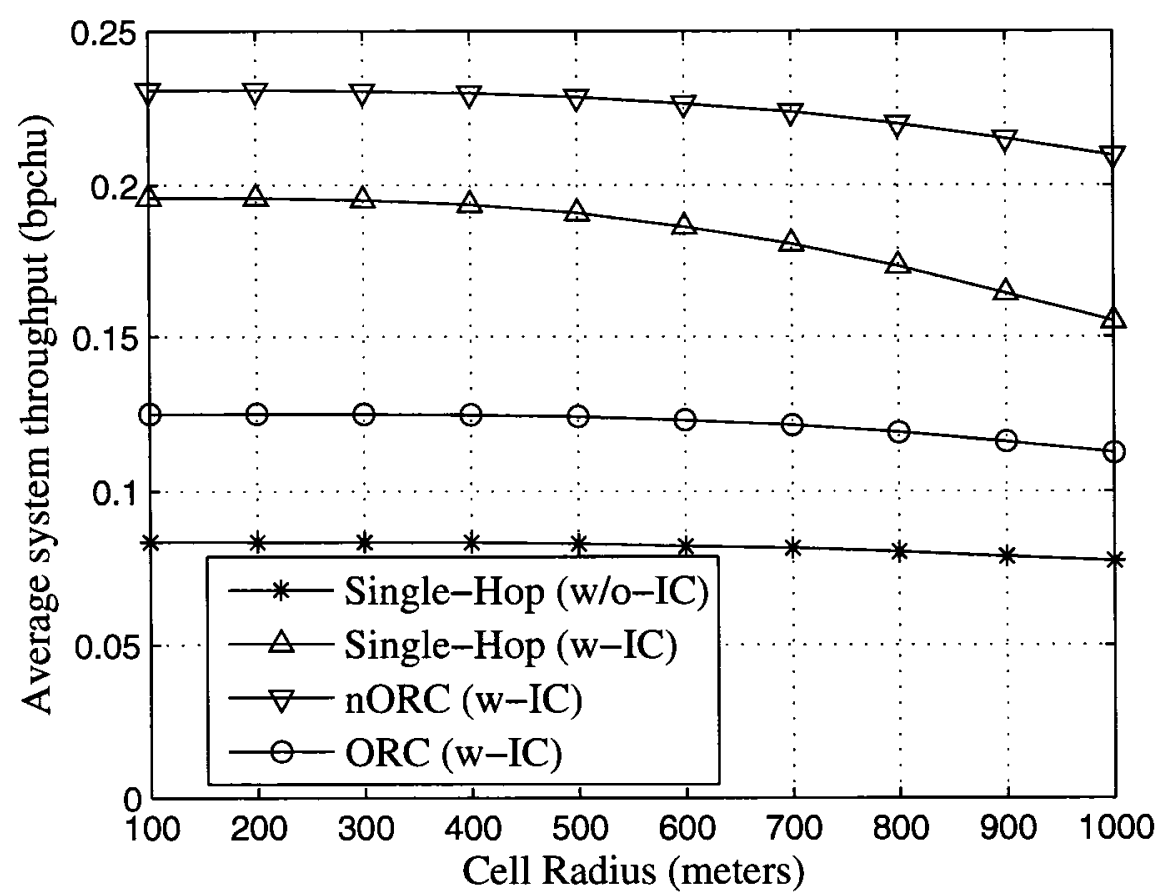

(a) Average system throughput

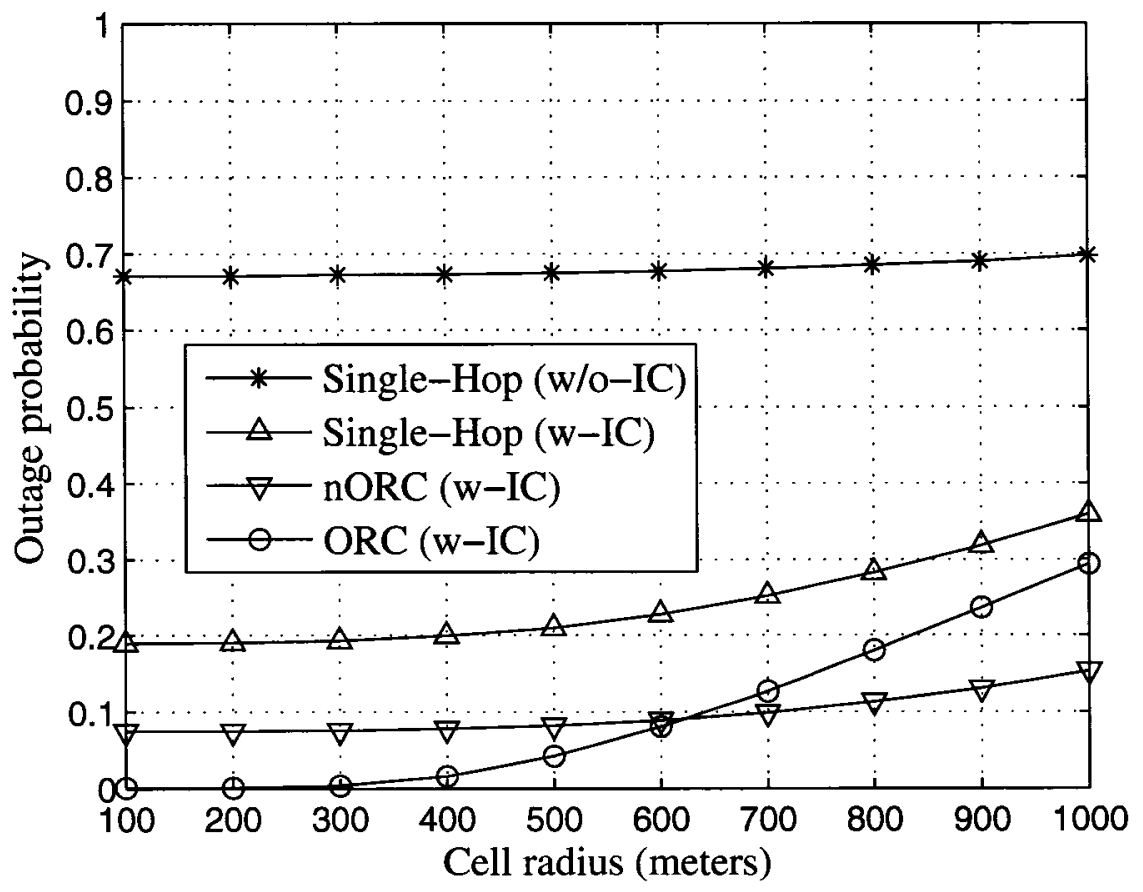

(b) Outage probability

Figure 4.28: System performance using IC, single vs. two-hop networks for $N_{\mathrm{CELL}}=7$, $N_{\mathrm{SEC}}=1$, and $C_{\mathrm{S}}=4$. 


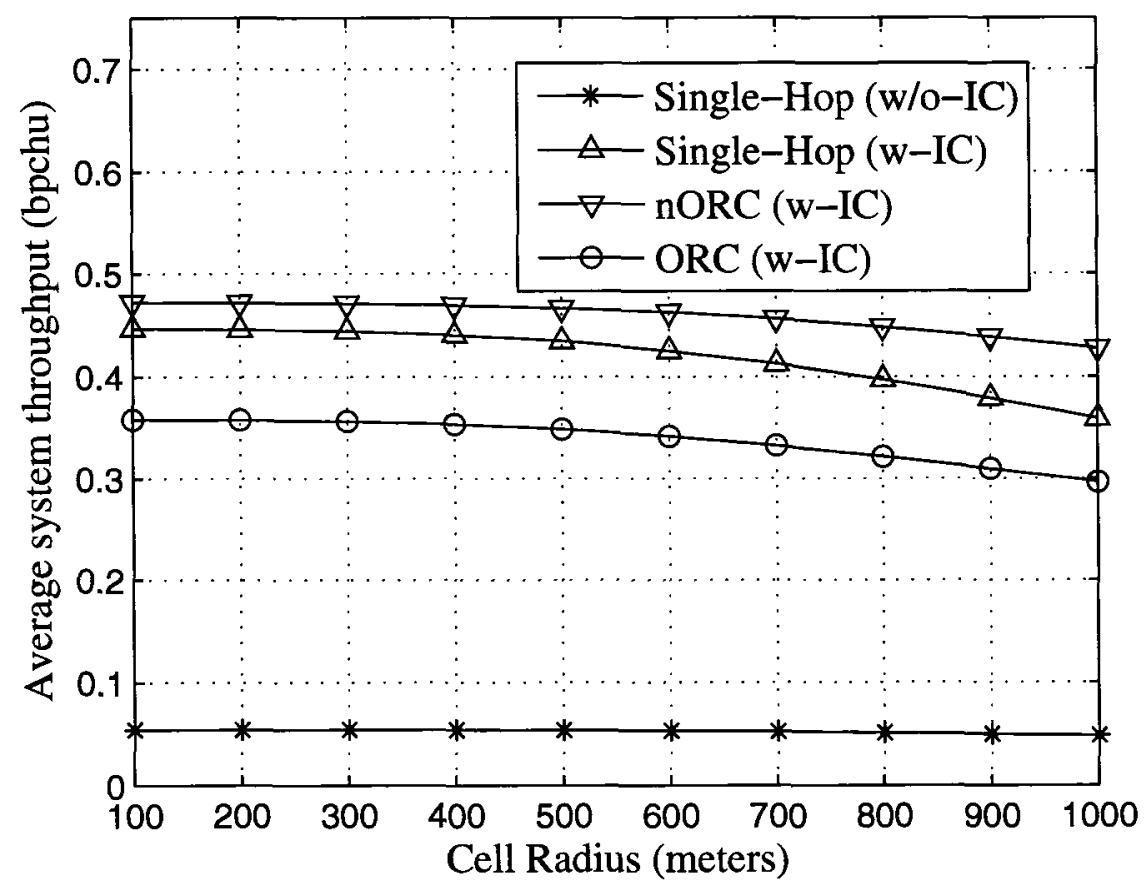

(a) Average system throughput

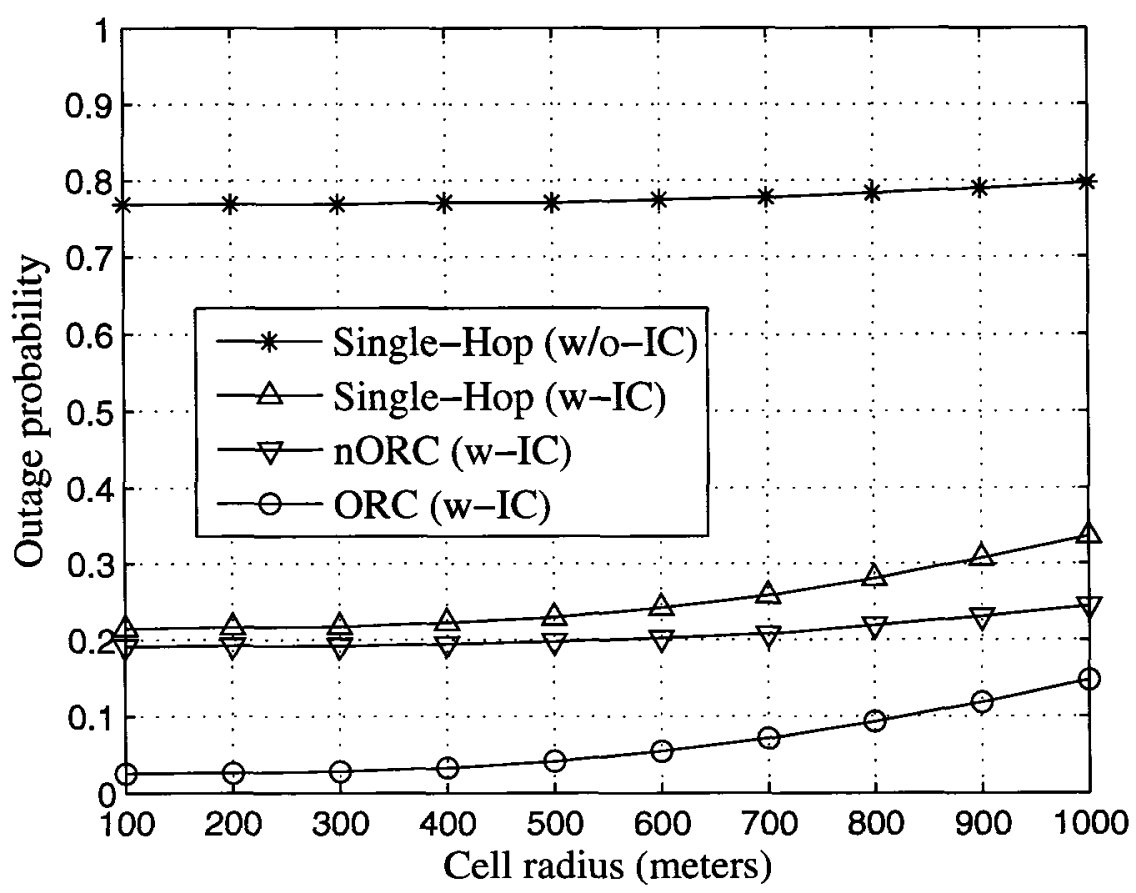

(b) Outage probability

Figure 4.29: System performance using IC, single vs. two-hop networks for $N_{\mathrm{CELL}}=7$, $N_{\mathrm{SEC}}=3$, and $C_{\mathrm{S}}=4$. 


\section{Chapter 5}

\section{Conclusions and Future Work}

\subsection{Conclusions}

In this thesis, we proposed using iterative MIMO multi-user detectors that use frequency domain filtering for co-channel interference (CCI) suppression in the downlink of single and two-hop wireless cellular systems. With a single receive antenna the MIMO receiver can be used to detect and efficiently cancel the major interferer in the system. Employing multiple antennas at the receiver not only gives diversity gain, but also allows the MIMO detector to efficiently cancel up to three interferers.

The system model used in this research to test and investigate the benefits of iterative interference cancellers (IC) in modern wireless system takes into account asynchronous interferers with different signal strengths through careful modelling of the wireless channel. The IC benefits were discussed for different cellular layouts including single-cell and multiple-cells scenarios and using different cluster sizes $\left(C_{\mathrm{S}}\right)$. Moreover, the system model investigated the use of single-sector cells $\left(N_{\mathrm{SEC}}=1\right)$ employing omnidirectional base-station antennas as well as bandwidth efficient threesectored cells $\left(N_{\mathrm{SEC}}=3\right)$ employing directional base-station antennas where all basestation sectors (BSSs) share the same frequency band. The IC performance has been compared to the perfect cancellation of the major interferer (PIC1). Also the range of possible improvement through more receive antennas has been investigated by 
studying the perfect cancellation of three major interferers (PIC3) instead of only one. In particular, the following benefits for using iterative ICs have been observed:

- Enabling the use of sectorized cells, where all sectors share the same bandwidth, to provide efficient utilization of resources while maintaining descent system outage performance. This is not possible without the IC due to the great deal of CCI introduced by the other BSSs in the same cell.

- Efficient CCI suppression from neighbouring cells in a multiple-cell scenario when using single-sector cells.

- Using the IC magnified the benefits that can be achieved from relay networks using ORCs, where there is a $50 \%$ reduction in the nominal system throughput due to the extra relay channel. Without the IC, relaying with ORCs had very minimal improvement over single-hop transmission especially in terms of the average system throughput.

- Additional benefits can be achieved from relaying with the IC when more relays are available per cell.

- The IC enabled relaying on non-orthogonal channels (nORCs) to improve the throughput performance of relay networks by removing the $50 \%$ reduction in the system nominal throughput, while maintaining their outage performance improvement. Without the IC using nORCs provides no or very little improvement over single-hop transmission.

- Using the IC with single-hop transmission outperforms relaying using ORCs in terms of throughput in both single-cell and four cells per cluster multiple-cell 
scenarios, with a reasonable outage performance that can be further improved if the MUs have multiple receive antennas.

- For a MU with a single receive antenna the IC provides a very close performance to the perfect cancellation of the major interferer (PIC1), which builds more confidence in the ability of the IC to achieve better results for MUs having two or more receive antennas close to the perfect cancellation of three major interferers (PIC3).

\subsection{Future Work}

The work presented in this thesis raises some interesting topics for future research.

- In this setup all interfering entities as well as the desired transmitter were assumed to use the same QPSK modulation scheme. The use of mixed modulation schemes for different transmitting entities and the effect of that on the IC performance should be addressed.

- This research was done for a single carrier system. Extending the same idea for OFDM systems would also be an important extension to this work.

- A more robust evaluation test bench is required for the multiple receive antennas case.

- Exploring the IC benefits with more advanced relaying techniques that incorporate cooperation among the different wireless entities, as well as exploring more than two-hops relaying scenarios. 
- Defining a cost function for the amount of power and time needed for iterative processing, and relating that to different environments having different constraints like wireless sensor networks.

- Utilizing more powerful error control coding, other than the conventional convolutional coding used in this work, such as turbo or LDPC codes.

- Perhaps the most important topic for future work, and the greatest limitation of the current study, is exploring the effect of channel estimation errors and developing suitable channel estimation techniques. This is particularly important for intermittent interferers, such as relayers. 


\section{References}

[1] S. Haykin, Communication Systems, 4th Edition. John Wiley \& Sons, Inc, 2004.

[2] T. S. Rappaport, Wireless Communications: Principles and Practice. Prentice Hall PTR, 2002.

[3] David Mottier and Loic Brunel, "Iterative space-time soft interference cancellation for UMTS-FDD uplink," IEEE Transactions on Vehicular Technology, vol. 52, no. 4, pp. 919-930, Jul 2003.

[4] L. Brunel and J. Boutros, "Code division multiple access based on independent codes and turbo decoding," Annales des Telecommunications, vol. 54, pp. 401410, Jul 1999.

[5] M.C. Reed and C.B. Schlegel and P.D. Alexander and J.A. Asenstorfer, "Iterative multiuser detection for CDMA with FEC: Near single user performance," IEEE Transactions on Communications, vol. 46, pp. 1693-1699, Dec 1998.

[6] S. Kapoor and S. Gollamudi and S. Magaraj and Y.F. Huang, "Adaptive multiuser detection and beamforming for interference suppression in CDMA mobile radio systems," IEEE Transactions on Communications, vol. 48, pp. 1341-1355, Sept 1999.

[7] H. Krim and M. Viberg, "Two decades of array signal processing research," IEEE Signal Proc. Mag., pp. 67-94, Jul 1996.

[8] S. Marinkovic and B. Vucetic and A. Ushirokawa, "Space-time iterative and multistage receiver structures for CDMA mobile communication systems," IEEE Journal on Seleted Areas in Communications, vol. 19, pp. 1594-1604, Aug 2001.

[9] J. Lin, "Iterative detection/equalization with frequency domain filtering for MIMO channels," Master's thesis, Carleton University, 2004.

[10] M.C. Reed and P.D. Alexander, "Iterative multiuser detection using antenna arrays and FEC on multipath channels," IEEE Journal on Seleted Areas in Communications, vol. 17, pp. 2082-2089, Dec 1999. 
[11] J. G. Proakis, Digital Communications. McGraw-Hill, 2001.

[12] T. Abe and T. Matsumoto, "Space-time turbo equalization in frequency-selective MIMO channels," IEEE Transactions on Vehicular Technology, vol. 52, pp. 469475, May 2003.

[13] M.V. Clark, "Adaptive frequency-domain equalization and diversity combining for broadband wireless communications," IEEE Journal on Seleted Areas in Communications, vol. 16, pp. 1385-1395, Oct 1998.

[14] D. Falconer and S.L. Ariyavisitakul and A. Benyamin-Seeyar and B. Eidson, "Frequency domain equalization for single-carrier broadband wireless system," IEEE Communications Magazine, vol. 40, pp. 58-66, April 2002.

[15] Y.D. Lin and Y.C. Hsu, "Multihop cellular: A new architecture for wireless communications," in IEEE INFOCOM'00, March 2000, pp. 1273-1282.

[16] M.O. Hasna and M. Alouini, "Performance analysis of two-hop relayed transmissions over Rayleigh fading channels," in IEEE 56th Vehicular Technology Conference (VTC'02), Sept 2002, pp. 1992-1996.

[17] H. Wu and C. Qiao and S. De and O. Tonguz, "Integrated cellular and ad hoc relaying systems: iCAR," IEEE Journal on Seleted Areas in Communications, vol. 19, no. 10, pp. 2105-2115, Oct 2001.

[18] H. Yanikomeroglu, "Fixed and mobile relaying technologies for cellular networks," in Second workshop on Applications and Services in Wireless Networks (ASWN'02), Jul 2002, pp. 75-81.

[19] H. Hu, "Performane analysis of cellular networks with digital fixed relays," Master's thesis, Carleton University, Sept 2003.

[20] D. Walsh, "Two-hop relaying in CDMA networks using the unlicensed bands," Master's thesis, Carleton University, Jan 2004.

[21] J. H. McClellan and C. M. Rader, Number Theory in Digital Signal Processing. Prentice Hall, 1979.

[22] L.R. Bahl and J. Cocke and F. Jelinek and J. Raviv, "Optimal decoding of linear codes for minimizing symbol error rate," IEEE Transactions on Information Theory, vol. 20, pp. 284-287, March 1974.

[23] V. Erceg and K.K.S. Hari and M.S. Smith and K.P. Sheikh and C. Tappenden and J.M. Costa and D.S. Baum and C. Bushue, "Channel models for fixed wireless applications," IEEE 802.16.Proposal 802.16.3c-01/29r4. 
[24] H. P. E. Stern and S. A. Mahmoud, Communication Systems, Analysis And Design. Pearson Prentice Hall, 2004.

[25] B. Sklar, Digital Communications, Fundamentals and Applications, Second Edition. Prentice Hall PTR, 2001.

[26] "https://www.ist-winner.org/."

[27] WINNER 2 WP1:, "Interim channel models," WINNER Deliverable D1.1.1 V1.1, Feb 2007.

[28] WINNER 2 WP6-System concept:, "Test scenarios and calibration cases issue 2," WINNER Deliverable D6.13.7 V1.00, Dec 2006.

[29] A. Pollard and D. Schultz and J. V. Hafen and M. Dottling and E. Zimmerman and R. Pabst, "WINNER - towards ubiquitous wireless access," in IEEE 63rd Vehicular Technology Conference, Spring 2006. 